\title{
A Metal-Free Entry to Phosphonylated Isoindoles by a Cascade of 5-exo-dig cyclization, [1,3]-Alkyl Shift and Aromatization Under Microwave Heating
}

Nicolai Dieltiens and Christian V. Stevens* 
Supporting Information Table of Contents

\begin{tabular}{|c|c|}
\hline Microwave reactions & 4 \\
\hline General remarks & 4 \\
\hline Synthesis of aminophosphonates 2a-m & 4 \\
\hline Synthesis of secondary amines iv & 5 \\
\hline Spectral data new secondary amines & $5-7$ \\
\hline Typical procedure for the synthesis of compounds 2 & 7 \\
\hline Spectral data $\mathbf{2 a}$ & 7 \\
\hline Spectral data $\mathbf{2 b}$ & 7 \\
\hline Spectral data 2c & 8 \\
\hline Spectral data 2d & 8 \\
\hline Spectral data 2e & 9 \\
\hline Spectral data $\mathbf{2 f}$ & 9 \\
\hline Spectral data $\mathbf{2 g}$ & 10 \\
\hline Spectral data $\mathbf{2 h}$ & 10 \\
\hline Spectral data $\mathbf{2 i}$ & 11 \\
\hline Spectral data $\mathbf{2} \mathbf{j}$ & 11 \\
\hline Spectral data $\mathbf{2 k}$ & 11 \\
\hline Spectral data 2l & 12 \\
\hline Spectral data $\mathbf{2 m}$ & 12 \\
\hline Typical procedure for the synthesis of isoindoles 4 and 14a-b & 13 \\
\hline Spectral data $\mathbf{4 a}$ & 13 \\
\hline Spectral data 4b & 13 \\
\hline Spectral data 4c & 14 \\
\hline Spectral data 4d & 14 \\
\hline Spectral data 4e & 14 \\
\hline Spectral data $\mathbf{4 f}$ & 15 \\
\hline Spectral data $\mathbf{4 g}$ & 15 \\
\hline Spectral data $4 \mathrm{~h}$ & 16 \\
\hline Spectral data 4i & 16 \\
\hline Spectral data 15a & 16 \\
\hline Spectral data $\mathbf{1 5 b}$ & 17 \\
\hline Synthesis of isoindole 13 & 17 \\
\hline Spectral data $\mathbf{1 3}$ & 17 \\
\hline Synthesis of azepino isoindole 16 & 18 \\
\hline Spectral data $\mathbf{1 6}$ & 18 \\
\hline Copy ${ }^{13}$ C-NMR 2a & 19 \\
\hline Copy ${ }^{1} \mathrm{H}$ NMR 2a & 20 \\
\hline Copy ${ }^{13}$ C-NMR 2b & 21 \\
\hline Copy ${ }^{1} \mathrm{H}$ NMR 2b & 22 \\
\hline Copy ${ }^{13}$ C-NMR 2c & 23 \\
\hline Copy ${ }^{1} \mathrm{H}$ NMR 2c & 24 \\
\hline Copy ${ }^{13}$ C-NMR 2d & 25 \\
\hline Copy ${ }^{1} \mathrm{H}$ NMR 2d & 26 \\
\hline Copy ${ }^{13}$ C-NMR 2e & 27 \\
\hline Copy ${ }^{1} \mathrm{H}$ NMR 2e & 28 \\
\hline Copy ${ }^{13}$ C-NMR 2f & 29 \\
\hline Copy ${ }^{1} \mathrm{H}$ NMR 2f & 30 \\
\hline
\end{tabular}




\begin{tabular}{|c|c|}
\hline Copy ${ }^{13}$ C-NMR 2g & 31 \\
\hline Copy ${ }^{1} \mathrm{H}$ NMR 2g & 32 \\
\hline Copy ${ }^{13}$ C-NMR $\mathbf{2 h}$ & 33 \\
\hline Copy ${ }^{1}$ H NMR 2h & 34 \\
\hline Copy ${ }^{13}$ C-NMR 2i & 35 \\
\hline Copy ${ }^{1}$ H NMR 2i & 36 \\
\hline Copy ${ }^{13}$ C-NMR 2j & 37 \\
\hline Copy ${ }^{1}$ H NMR $\mathbf{2 j}$ & 38 \\
\hline Copy ${ }^{13}$ C-NMR 2k & 39 \\
\hline Copy ${ }^{1}$ H NMR 2k & 40 \\
\hline Copy ${ }^{13}$ C-NMR 2l & 41 \\
\hline Copy ${ }^{1}$ H NMR 2l & 42 \\
\hline Copy ${ }^{13}$ C-NMR 2m & 43 \\
\hline Copy ${ }^{1}$ H NMR 2m & 44 \\
\hline Copy ${ }^{13}$ C-NMR 4a & 45 \\
\hline Copy ${ }^{1}$ H NMR 4a & 46 \\
\hline Copy ${ }^{13}$ C-NMR 4b & 47 \\
\hline Copy ${ }^{1} \mathrm{H}$ NMR 4b & 48 \\
\hline Copy ${ }^{13}$ C-NMR 4c & 49 \\
\hline Copy ${ }^{1} \mathrm{H}$ NMR 4c & 50 \\
\hline Copy ${ }^{13}$ C-NMR 4d & 51 \\
\hline Copy ${ }^{1}$ H NMR 4d & 52 \\
\hline Copy ${ }^{13}$ C-NMR 4e & 53 \\
\hline Copy ${ }^{1}$ H NMR 4e & 54 \\
\hline Copy ${ }^{13}$ C-NMR $4 f$ & 55 \\
\hline Copy ${ }^{1} \mathrm{H}$ NMR 4f & 56 \\
\hline Copy ${ }^{13}$ C-NMR 4g & 57 \\
\hline Copy ${ }^{1}$ H NMR 4h & 58 \\
\hline Copy ${ }^{13}$ C-NMR 4h & 59 \\
\hline Copy ${ }^{1}$ H NMR 4h & 60 \\
\hline Copy ${ }^{13}$ C-NMR 4i & 61 \\
\hline Copy ${ }^{1}$ H NMR 4i & 62 \\
\hline Copy ${ }^{13}$ C-NMR 14a & 63 \\
\hline Copy ${ }^{1}$ H NMR 14a & 64 \\
\hline Copy ${ }^{13}$ C-NMR 14b & 65 \\
\hline Copy ${ }^{1} \mathrm{H}$ NMR 14b & 66 \\
\hline Copy ${ }^{13}$ C-NMR 13 & 67 \\
\hline Copy ${ }^{1}$ H NMR 13 & 68 \\
\hline Copy ${ }^{13}$ C-NMR 16 & 69 \\
\hline Copy ${ }^{1}$ H NMR 16 & 70 \\
\hline
\end{tabular}


Microwave reactions: all microwave reactions were performed in the CEM Focused Microwave $^{T M}$ Synthesis System, Model Discover, with a selectable power output from 0-300 watts. The reactions were performed in 10-mL thick walled Pyrex reaction vessels closed with a Septa cap and equiped with a small stirring bar. The temperature control system uses a noncontact infrared sensor to measure temperature on the bottom of the vessel and is used in a feedback loop with the on-board computer to regulate the temperature from $25-250^{\circ} \mathrm{C}$ by adjusting the power output (1-watt increments). The pressure control, IntelliVent ${ }^{T M}$ Pressure Control System, uses an indirect measurement of the pressure by sensing changes in the external deflection of the septa on the top of the sealed pressure vessel. Stirring is performed by a rotating magnetic plate located below the floor of the microwave cavity. Cooling of the vessel after the reaction is performed by a stream of clean air onto the vessel which decreases the temperature of a $2 \mathrm{~mL}$ solution from $\sim 150^{\circ} \mathrm{C}$ to $\sim 40^{\circ} \mathrm{C}$ in less then 120 seconds. A ramp time of maximum 5 minutes is used during which the temperature increases from RT to the desired temperature. This temperature is maintained during the course of the reaction for the indicated time.

General remarks: High resolution ${ }^{1} \mathrm{H}-\mathrm{NMR}(300 \mathrm{MHz})$ and ${ }^{13} \mathrm{C}-\mathrm{NMR}$ (75 MHz) spectra were run on a Jeol JNM-EX 300 NMR. Peak assignments were obtained with the aid of DEPT, 2D-HSQC, 2D-COSY spectra. The compounds were diluted in deuterated solvents and the used solvent is indicated for each compound. Low resolution mass spectra were recorded on an Agilent 1100 Series VS (ES, 4000V) mass spectrometer. IR-spectra were obtained from a Perkin Elmer Spectrum One infrared spectrometer. For liquid samples the spectra were collected by preparing a thin film of compound between two sodium chloride plates. The crystalline compounds were mixed with potassium bromide and pressed until a transparent potassium bromide plate was obtained. Melting points of crystalline compounds were measured with a Büchi 540 apparatus. The purification of reaction mixtures was performed by flash chromatography using a glass column with silica gel (Across, particle size 0.035-0.070 mm, Pore diameter ca. $6 \mathrm{~nm}$ ).

Synthesis of aminophosphonates 2a-m: compounds $\mathbf{2 a - m}$ were synthesized using a three component coupling between secondary amines iv, 2-ethynylbenzaldehyde and $\mathrm{P}(\mathrm{OMe})_{3}$ mediated by $\mathrm{LiClO}_{4}$ in diethylether as depicted in Scheme $\mathbf{i}$ and described by Azizi and coworkers (Azizi, N.; Saidi, M. R. Tetrahedron 2003, 59, 5329-5332). 
Synthesis of secondary amines iv: All secondary amines were synthesized using a reductive amination, except those used for the synthesis of $\mathbf{2 1 - \mathbf { m }}$ who are commercially available. A suitable aldehyde $\mathbf{i}$ was dissolved in dry $\mathrm{CH}_{2} \mathrm{Cl}_{2}$ (freshly distilled from $\mathrm{CaH}_{2}$ ) and 1 equivalent of amine ii and 2 equivalents of $\mathrm{MgSO}_{4}$ were added. The mixture was allowed to stir at room temperature for 24 hours. After filtration of the solids and removal of the volatiles, the obtained aldimines iii were dissolved in dry $\mathrm{MeOH}$. To this solution 1.1 equivalent of $\mathrm{NaBH}_{4}$ was carefully added and stirring was continued for 4 hours. The reaction was quenched by the addition of $\mathrm{NaHCO}_{3}$ (sat, aq) and the $\mathrm{MeOH}$ was removed under reduced pressure. The residue was extracted with $\mathrm{CH}_{2} \mathrm{Cl}_{2}$ and dried using $\mathrm{MgSO}_{4}$. After filtration of the solids and removal of the volatiles, the obtained amines iv were directly used for the synthesis of compounds 2 .

Scheme i

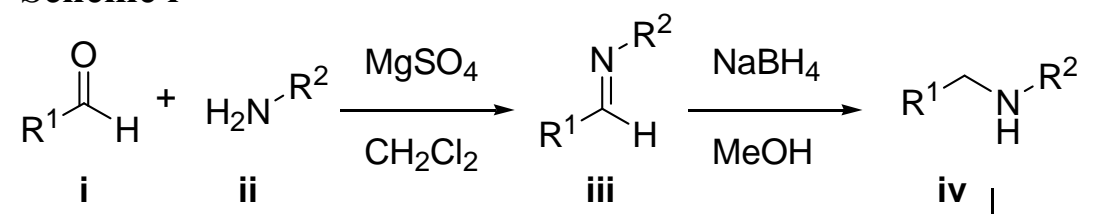<smiles>C#Cc1ccccc1C=O</smiles><smiles>[R]CN([R])C([Po])c1ccccc1C#C</smiles>

2a-m

\section{N-[(2E)-3-(4-methoxyphenyl)prop-2-enyl]-N-propylamine}<smiles>CCCNC/C=C/c1ccc(OC)cc1</smiles>

${ }^{1}$ H-NMR (300 MHz, $\left.\mathbf{C D C l}_{3}\right): \boldsymbol{\delta} 0.93\left(\mathrm{t}, J=7.3 \mathrm{~Hz}, 3 \mathrm{H}, \mathrm{CH}_{3}\right)$, 1.55 (sextet, $J=7.3 \mathrm{~Hz}, 2 \mathrm{H}, \mathrm{CH}_{2} \mathrm{CH}_{3}$ ), 1.94 (s, $1 \mathrm{H}, \mathrm{NH}$ ), 2.63 (t, $J=7.3 \mathrm{~Hz}, 2 \mathrm{H}, \mathrm{NC}_{2} \mathrm{CH}_{2}$ ), 3.40 (dd, $J=1.2 \mathrm{~Hz}, J=6.5 \mathrm{~Hz}$, $\left.2 \mathrm{H}, \mathrm{NC}_{2} \mathrm{CH}\right), 3.80\left(\mathrm{~s}, 3 \mathrm{H}, \mathrm{PhOCH}_{3}\right), 6.18(\mathrm{dt}, J=15.7 \mathrm{~Hz}, J=6.5 \mathrm{~Hz}, 1 \mathrm{H}, \underline{\mathrm{HC}}=\mathrm{CHPh})$, 6.47 (d, $J=15.7 \mathrm{~Hz}, 1 \mathrm{H}, \underline{\mathrm{HCPh}}), 6.84$ (d, $J=8.8 \mathrm{~Hz}, 2 \mathrm{H}, 2 \times \mathrm{CH}_{\text {arom }}$ ), 7.31 (d, $J=8.5 \mathrm{~Hz}$, $\left.2 \mathrm{H}, 2 \times \mathrm{CH}_{\text {arom }}\right) .{ }^{13} \mathbf{C}-\mathbf{N M R}$ (75 MHz, $\left.\mathbf{C D C l} 3\right): \delta 11.86\left(\mathrm{CH}_{2} \mathrm{CH}_{3}\right), 23.28\left(\mathrm{CH}_{2} \mathrm{CH}_{3}\right), 51.40$ $\left(\mathrm{NCH}_{2} \mathrm{CH}_{2}\right), 52.03\left(\mathrm{NCH}_{2} \mathrm{CH}\right), 55.04\left(\mathrm{OCH}_{3}\right), 113.91$ (2 x CHarom), $126.41(\mathrm{HC}=\mathrm{CHPh})$, 127.36 (2 x CH $\left.\mathrm{CH}_{\text {arom }}\right), 129.96\left(\mathrm{C}_{\mathrm{q}, \text { arom }}\right), 130.54(\mathrm{HC}=\mathrm{CHPh}), 159.01\left(\mathrm{C}_{\mathrm{q}}, \mathrm{Ph}\right) . \mathbf{I R}\left(\mathbf{c m}^{-1}\right) \mathbf{v}_{\text {max }}$ : 1608 (C=C), 3300 (br NH). MS (ESI): m/z (\%): No M+H $\mathrm{H}^{+}, 147.2\left(\mathrm{M}^{+}-\mathrm{NC}_{3} \mathrm{H}_{8}, 100\right)$.

\section{N-(4-methylbenzyl)-N-(3-methylbut-2-enyl)amine}

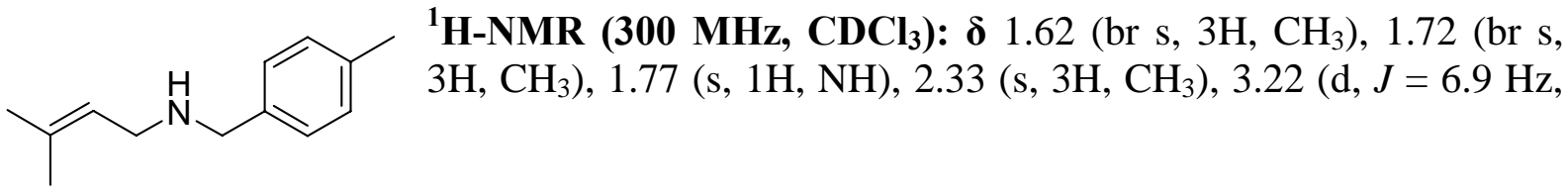


$\mathrm{NC}_{2} \mathrm{CH}$ ), 3.74 (s, $2 \mathrm{H}, \mathrm{NC}_{2} \mathrm{C}$ ), 5.28 (t x septet, $J=6.9 \mathrm{~Hz}, J=1.4 \mathrm{~Hz}, 1 \mathrm{H}, \mathrm{NCH}_{2} \mathrm{C} \underline{\mathrm{H}}$ ), 7.11-7.26 (m, 4H, $\left.4 \times \mathrm{CH}_{\text {arom }}\right) .{ }^{13} \mathbf{C}-\mathbf{N M R}\left(\mathbf{7 5} \mathbf{M H z}, \mathbf{C D C l}_{3}\right): \boldsymbol{\delta} 14.16\left(\mathrm{CH}_{2} \mathrm{CH}_{3}\right), 16.73$ $\left(\mathrm{CCH}_{3}\right), 20.65\left(\underline{\mathrm{CH}}_{2} \mathrm{CH}_{3}\right), 32.44\left(\mathrm{NCH}_{2} \underline{\mathrm{CH}}_{2}\right), 49.10\left(\mathrm{NCH}_{2} \mathrm{CH}_{2}\right), 58.35\left(\mathrm{NCH}_{2} \mathrm{C}\right), 125.51$ ( $\underline{\mathrm{CH}} \mathrm{H} h), 126.20$ ( $\left.\mathrm{CH}_{\text {arom }}\right), 128.14$ (2 x CH arom $), 128.96$ (2 x CHarom), $137.33\left(\mathrm{CCH}_{3}\right), 138.20$

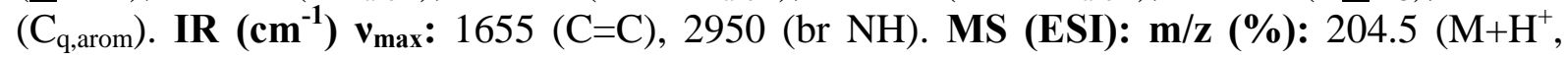
100).

\section{$\mathrm{N}-[(2 \mathrm{E})-4-m e t h y l p e n t-2-e n y l]-N-(2-p h e n y l e t h y l)$ amine}

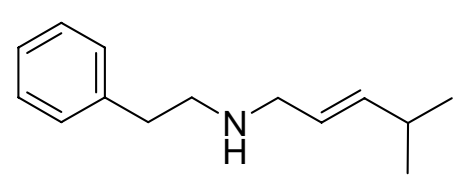

${ }^{1}$ H-NMR (300 MHz, CDCl 3 ): $\boldsymbol{\delta} 0.97$ (d, $J=6.8 \mathrm{~Hz}, 6 \mathrm{H}, 2 \mathrm{x}$ $\mathrm{CH}_{3}$ ), 1.30 (br s, $1 \mathrm{H}, \mathrm{NH}$ ), 2.26 (octet, $J=6.8 \mathrm{~Hz}, 1 \mathrm{H}, \mathrm{CH}$ ), 2.78$2.90\left(\mathrm{~m}, 4 \mathrm{H}, \mathrm{CH}_{2} \mathrm{CH}_{2}\right), 3.19\left(\mathrm{~d}, J=5.8 \mathrm{~Hz}, 2 \mathrm{H}, \mathrm{NC}_{2} \mathrm{CH}\right), 5.39-$ $5.58(\mathrm{~m}, 2 \mathrm{H}, \underline{\mathrm{HC}}=\mathrm{CHPh}), 7.17-7.32\left(\mathrm{~m}, 5 \mathrm{H}, 5 \times \mathrm{CH}_{\mathrm{arom}}\right) .{ }^{13} \mathrm{C}-$ NMR (75 MHz, CDCl $\left.)_{3}\right): \delta 22.51\left(2 \times \mathrm{CH}_{3}\right), 30.91\left(\underline{\mathrm{CH}}\left(\mathrm{CH}_{3}\right)_{2}\right), 36.53\left(\mathrm{CH}_{2} \mathrm{Ph}\right), 50.65$ $\left(\mathrm{NCH}_{2} \mathrm{CH}_{2}\right), 51.75\left(\mathrm{NCH}_{2} \mathrm{CH}\right), 125.24(\mathrm{HC}=\mathrm{CHPh}), 126.18\left(\mathrm{CH}_{\text {arom }}\right), 128.38\left(2 \times \mathrm{CH}_{\text {arom }}\right)$, 128.80 (2 x CH arom), $139.73(\mathrm{HC}=\underline{\mathrm{C} H P h}), 140.22\left(\mathrm{C}_{\mathrm{q}}, \mathrm{Ph}\right) . \mathbf{I R}\left(\mathbf{c m}^{-1}\right) \mathbf{v}_{\max }: 1604(\mathrm{C}=\mathrm{C}), 2958$ (br NH). MS (ESI): m/z (\%): $204.5\left(\mathrm{M}+\mathrm{H}^{+}, 100\right)$.

\section{Allyl-(3-fluorobenzyl)amine}

${ }^{1} \mathbf{H}-\mathbf{N M R}\left(\mathbf{3 0 0} \mathbf{M H z}, \mathbf{C D C l}_{3}\right): \boldsymbol{\delta} 1.53(\mathrm{br}, 1 \mathrm{H}, \mathrm{NH}), 3.27(\mathrm{dt}, J=1.2$
$\left.\mathrm{Hz}, J=6.1 \mathrm{~Hz}, 2 \mathrm{H}, \mathrm{NCH}_{2} \mathrm{CH}\right), 3.79\left(\mathrm{~s}, 2 \mathrm{H}, \mathrm{NCH}_{2} \mathrm{Ph}\right), 5.31(\mathrm{dq}, J=$ $\mathrm{Hz}, 1 \mathrm{H}, \mathrm{HC}=\mathrm{CH}_{\mathrm{A}} \underline{\mathrm{H}}_{\mathrm{B}}$ ), $5.92\left(\mathrm{ddt}, J=6.1 \mathrm{~Hz}, J=10.2 \mathrm{~Hz}, J=17.1 \mathrm{~Hz}, 1 \mathrm{H}, \underline{\mathrm{HC}}=\mathrm{CH}_{2}\right.$ ), 6.917.32 (m, 4H, $\left.4 \times \mathrm{CH}_{\text {arom }}\right) .{ }^{13} \mathbf{C}-\mathbf{N M R}\left(\mathbf{7 5} \mathbf{M H z}, \mathbf{C D C l}_{3}\right): \delta 51.74\left(\mathrm{NC}_{2} \mathrm{CH}\right), 52.65$ $\left(\mathrm{NCH}_{2} \mathrm{Ph}\right), 113.83$ (d, $\left.J=21.9 \mathrm{~Hz}, \mathrm{CH}_{\text {arom }}\right), 114.96$ (d, $\left.J=20.8 \mathrm{~Hz}, \mathrm{CH}_{\text {arom }}\right), 116.21$ $\left(\mathrm{HC}=\underline{C H}_{2}\right), 123.70$ (d, $\left.J=3.5 \mathrm{~Hz}, \mathrm{CH}_{\text {arom }}\right), 129.86\left(\mathrm{~d}, J=8.1 \mathrm{~Hz}, \mathrm{CH}_{\text {arom }}\right), 136.67\left(\mathrm{HC}=\mathrm{CH}_{2}\right)$, 143.12 (d, $\left.J=6.9 \mathrm{~Hz}, \mathrm{CH}_{\mathrm{q}, \text { arom }}\right), 163.08$ (d, $\left.J=245.8 \mathrm{~Hz}, \mathrm{FC}_{\mathrm{q}, \text { arom}}\right) .{ }^{\mathbf{1 9}}$ F-NMR (282 MHz, $\left.\mathbf{C D C l}_{3}\right): \boldsymbol{\delta}-113.41$ (dd, $\left.J=9.8 \mathrm{~Hz}, J=16.8 \mathrm{~Hz}\right) . \mathbf{I R}\left(\mathbf{c m}^{-1}\right) \mathbf{v}_{\max }: 1590(\mathrm{C}=\mathrm{C}), 1616(\mathrm{C}=\mathrm{C})$, 1644 (C=C), 2823 (br NH). MS (ESI): m/z (\%): $166.3\left(\mathrm{M}+\mathrm{H}^{+}, 100\right)$.

\section{N-(4-methylbenzyl)-N-(3-methylbut-2-enyl)amine}<smiles>Clc1ccc(CCNCC2=CCCCC2)cc1</smiles>

${ }^{1} \mathbf{H}-N M R\left(300 ~ M H z, \mathbf{C D C l}_{3}\right): \delta 1.48$ (br s, $\left.1 \mathrm{H}, \mathrm{NH}\right), 1.52-1.67$ (m, $\left.4 \mathrm{H}, \mathrm{CH}_{2} \mathrm{CH}_{2}\right), 1.87-1.94\left(\mathrm{~m}, 2 \mathrm{H}, \mathrm{CC}_{2}\right), 1.96-2.05(\mathrm{~m}, 2 \mathrm{H}$, $\left.\mathrm{HCC}_{2}\right), 2.74-2.84\left(\mathrm{~m}, 4 \mathrm{H}, \mathrm{NCH}_{2} \mathrm{CH}_{2}\right), 3.11$ (s, $\left.2 \mathrm{H}, \mathrm{NCH}_{2}\right)$, 5.54 (br s, HC), 7.14 (d, $\left.2 \mathrm{H}, J=8.4 \mathrm{~Hz}, 2 \mathrm{H}, 2 \times \mathrm{CH}_{\text {arom}}\right), 7.26$ (d, 2H, J = 8.4 Hz, 2H, $\left.2 \times \mathrm{CH}_{\text {arom }}\right) .{ }^{13} \mathbf{C}-\mathbf{N M R}(75 \mathrm{MHz}$, $\left.\mathbf{C D C l}_{3}\right): \boldsymbol{\delta} 22.61\left(\mathrm{CH}_{2}\right), 22.82\left(\mathrm{CH}_{2}\right), 25.14\left(\mathrm{HCCH}_{2}\right), 26.96\left(\mathrm{CCH}_{2}\right), 35.81\left(\underline{\mathrm{CH}}_{2} \mathrm{Ph}\right), 50.32$ $\left(\mathrm{NCH}_{2} \mathrm{CH}_{2}\right), 56.13\left(\mathrm{NCH}_{2} \mathrm{C}\right), 122.86(\mathrm{HC}=\mathrm{C}), 128.61$ (2x CH $131.93\left(\mathrm{ClC}_{\mathrm{q}, \text { arom }}\right), 135.97\left(\mathrm{C}_{\mathrm{q}, \text { arom }}\right), 138.77(\underline{\mathrm{C}}=\mathrm{CH}) . \mathbf{I R}\left(\mathbf{c m}^{-1}\right) \mathbf{v}_{\max }$ : $1670(\mathrm{C}=\mathrm{C}), 2925(\mathrm{NH})$.

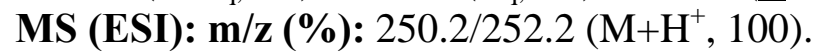

\section{N-butyl-N-[(2E)-2-methyl-3-phenylprop-2-enyl]amine}

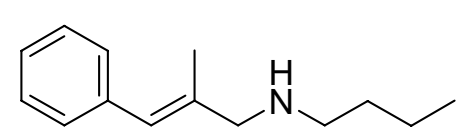

${ }^{1}$ H-NMR (300 MHz, $\left.\mathbf{C D C l}_{3}\right)$ : $\boldsymbol{\delta} 0.93\left(\mathrm{t}, J=7.3 \mathrm{~Hz}, 3 \mathrm{H}, \mathrm{CH}_{3}\right)$, 1.36 (s, $1 \mathrm{H}, \mathrm{NH}$ ), 1.37 (sextet, $J=7.3 \mathrm{~Hz}, 2 \mathrm{H}, \mathrm{CH}_{2} \mathrm{CH}_{3}$ ), 1.471.57 (m, $\left.2 \mathrm{H}, \mathrm{NCH}_{2} \mathrm{CH}_{2}\right), 1.89$ (d, $\left.J=1.1 \mathrm{~Hz}, 3 \mathrm{H}, \mathrm{CH}_{3}\right), 2.63$ (t, $J$ $=7.2 \mathrm{~Hz}, \mathrm{NC}_{2} \mathrm{CH}_{2}$ ), 3.32 (s, $2 \mathrm{H}, \mathrm{NC}_{2} \mathrm{C}$ ), 6.34 (br s, $1 \mathrm{H}, \mathrm{C} \underline{\mathrm{HPh}}$ ), 7.17-7.35 (m, 5H, $5 \mathrm{x}$ $\left.\mathrm{CH}_{\text {arom }}\right) .{ }^{13} \mathbf{C}-\mathbf{N M R}\left(75 \mathrm{MHz}, \mathbf{C D C l}_{3}\right): \boldsymbol{\delta} 14.16\left(\mathrm{CH}_{2} \underline{\mathrm{CH}_{3}}\right), 16.73\left(\mathrm{C}^{\mathrm{C}} \mathrm{H}_{3}\right), 20.65\left(\underline{\mathrm{CH}}_{2} \mathrm{CH}_{3}\right)$, 
$32.44\left(\mathrm{NCH}_{2} \mathrm{CH}_{2}\right), 49.10\left(\mathrm{NCH}_{2} \mathrm{CH}_{2}\right), 58.35\left(\mathrm{NC}_{2} \mathrm{C}\right), 125.51(\underline{\mathrm{CHPh}}), 126.20\left(\mathrm{CH}_{\text {arom }}\right)$, 128.14 (2 x CHarom), 128.96 (2 x CHarom), $137.33\left(\mathrm{C} \mathrm{CH}_{3}\right), 138.20\left(\mathrm{C}_{\mathrm{q}, \text { arom }}\right) . \mathbf{I R}\left(\mathbf{c m}^{-1}\right) \mathbf{v}_{\text {max }}$ : 1655 (C=C), 2950 (br NH). MS (ESI): m/z (\%): $204.5\left(\mathrm{M}+\mathrm{H}^{+}, 100\right)$.

\section{Typical procedure for the synthesis of compounds 2.}

In a dry flask, 2-ethynylbenzaldehyde $(3.84 \mathrm{mmol})$ is dissolved into diethylether $(6 \mathrm{~mL}$, freshly distilled from Na-metal). To this solution is added $\mathrm{LiClO}_{4}$ (7.5 equiv, $28.8 \mathrm{mmol}$, dried for $24 \mathrm{~h}$ at $110^{\circ} \mathrm{C}$ ). This mixture is stirred for 5 minutes. Subsequently secondary amine iv is added (2 equiv, $7.69 \mathrm{mmol}$, dissolved in $1 \mathrm{~mL}$ dry diethylether). This mixture is stirred for 20 minutes after which $\mathrm{P}(\mathrm{OMe})_{3}$ is added (1.5 equiv, $\left.5.76 \mathrm{mmol}\right)$. The reaction is stirred for 30 minutes after which water is very carefully added $(20 \mathrm{~mL})$. The mixture is extracted with $\mathrm{CH}_{2} \mathrm{Cl}_{2}$ (3 x 20mL) and dried using $\mathrm{MgSO}_{4}$. After filtration of the solids and removal of the volatiles, the obtained compounds were purified using either crystallization, column chromatography or acid/base extraction.

\section{Dimethyl [benzyl-(3-phenylprop-2-enyl)amino](2-ethynylphenyl)methylphosphonate 2a}

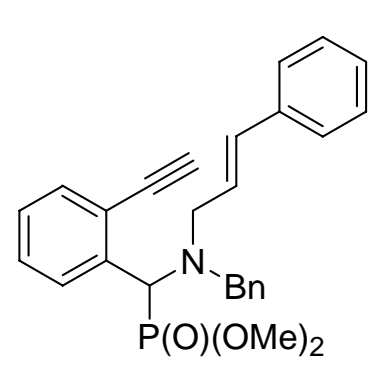

${ }^{1}$ H-NMR (300 MHz, $\mathbf{C D C l}_{3}$ ): $\boldsymbol{\delta} 3.01$ (s, 1H, CCH), 3.20 (dd, $J=7.4$ $\left.\mathrm{Hz}, J=14.6 \mathrm{~Hz}, 1 \mathrm{H}, \mathrm{NCH}_{\mathrm{A}} \mathrm{H}_{\mathrm{B}} \mathrm{CH}\right), 3.45\left(\mathrm{~d}, J=10.5 \mathrm{~Hz}, 3 \mathrm{H}, \mathrm{OCH}_{3}\right)$, 3.63 (d, $J=14.0 \mathrm{~Hz}, 1 \mathrm{H}, \mathrm{NCH}_{\mathrm{A}} \mathrm{H}_{\mathrm{B}} \mathrm{Ph}$ ), 3.75 (ddt, $J=2.5 \mathrm{~Hz}, J=5.2$ $\left.\mathrm{Hz}, J=14.6 \mathrm{~Hz}, 1 \mathrm{H}, \mathrm{NCH}_{\mathrm{A}} \underline{\mathrm{H}}_{\mathrm{B}} \mathrm{CH}\right), 3.91$ (d, $\left.J=10.5 \mathrm{~Hz}, 3 \mathrm{H}, \mathrm{OCH}_{3}\right)$, $4.33\left(\mathrm{~d}, J=14.2 \mathrm{~Hz}, 1 \mathrm{H}, \mathrm{NCH}_{\mathrm{A}} \underline{\mathrm{H}}_{\mathrm{B}} \mathrm{Ph}\right), 5.12(\mathrm{~d}, J=24.0 \mathrm{~Hz}, 1 \mathrm{H}$, CHP), 6.18 (ddd, $J=15.7 \mathrm{~Hz}, J=7.4 \mathrm{~Hz}, J=5.2 \mathrm{~Hz}, 1 \mathrm{H}, \underline{\mathrm{HC}}=\mathrm{CHPh}$ ), 6.48 (d, $J=15.7 \mathrm{~Hz}, 1 \mathrm{H}, \underline{\mathrm{HCPh}}), 7.17-7.58\left(\mathrm{~m}, 13 \mathrm{H}, 13 \times \mathrm{CH}_{\text {arom }}\right)$, 8.00 (d, $J=8.0 \mathrm{~Hz}, 1 \mathrm{H}$, PCHCCC트). ${ }^{13} \mathbf{C}-\mathbf{N M R}\left(75 \mathbf{M H z}, \mathbf{C D C l}_{3}\right): \delta$ $53.04\left(\mathrm{~d}, J=6.9 \mathrm{~Hz}, \mathrm{OCH}_{3}\right), 53.63\left(\mathrm{~d}, J=6.9 \mathrm{~Hz}, \mathrm{OCH}_{3}\right), 53.70$ (d, $J=8.1 \mathrm{~Hz}, \mathrm{NCH}_{2} \mathrm{CH}$ ), 55.48 (d, $J=6.9 \mathrm{~Hz}, \mathrm{NCCH}_{2} \mathrm{Ph}$ ), 58.85 (d, $\left.J=160.1 \mathrm{~Hz}, \mathrm{CHP}\right), 82.11(\underline{\mathrm{CCH}}), 82.17$ (Cㅡㅏ), 124.15 (d, $J=12.7 \mathrm{~Hz}$, PCHCC $), 126.34$ (2 x CHarom), $126.86\left(\mathrm{CH}_{\text {arom }}\right), 127.35\left(\mathrm{CH}_{\text {arom }}\right)$, $127.70(\mathrm{HC}=\mathrm{CHPh}), 128.11\left(\mathrm{CH}_{\text {arom }}\right), 128.18$ (2 x CHarom), $128.57\left(3 \times \mathrm{CH}_{\text {arom }}\right), 128.72$ (3 x $\left.\mathrm{CH}_{\text {arom }}\right), 130.73$ (d, $\left.J=3.5 \mathrm{~Hz}, \mathrm{PCHC} \underline{\mathrm{CH}}\right), 132.58(\underline{\mathrm{CHPh}}), 133.45$ (PCHCCCH $), 135.98$ (d, $J$ $=5.8 \mathrm{~Hz}, \mathrm{PCHC}), 137.38\left(\mathrm{C}_{\mathrm{q}}, \mathrm{Ph}\right), 140.00\left(\mathrm{C}_{\mathrm{q}}, \mathrm{Ph}\right) .{ }^{31}$ P-NMR (121 MHz, CDCl $\left.\mathbf{3}\right): \boldsymbol{\delta} 26.47$. IR $\left(\mathbf{c m}^{-1}\right) \mathbf{v}_{\text {max }}: 1032$ (P-O), $1056(\mathrm{P}-\mathrm{O}), 1248(\mathrm{P}=\mathrm{O}), 1641(\mathrm{C}=\mathrm{C}), 2090$ (alkyne). MS (ESI): m/z (\%): $446.3\left(\mathrm{M}+\mathrm{H}^{+}, 100\right)$. Chromatography: Hex/EtOAc 4/6 $\mathrm{R}_{\mathrm{f}}=0.18$. Yield: $68 \%$.

\section{Dimethyl [allyl(benzyl)amino](2-ethynylphenyl)methylphosphonate 2b}

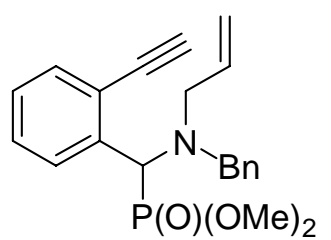

${ }^{1}$ H-NMR (300 MHz, $\mathbf{C D C l}_{3}$ ): $\boldsymbol{\delta} 3.04$ (s, 1H, CCH), 3.06 (dd, $J=7.2$ $\left.\mathrm{Hz}, J=14.6 \mathrm{~Hz}, 1 \mathrm{H}, \mathrm{NCH}_{\mathrm{A}} \mathrm{H}_{\mathrm{B}} \mathrm{CH}\right), 3.44\left(\mathrm{~d}, J=10.5 \mathrm{~Hz}, 3 \mathrm{H}, \mathrm{OCH}_{3}\right)$, 3.52 (d, $J=14.2 \mathrm{~Hz}, 1 \mathrm{H}, \mathrm{NC}_{\mathrm{H}_{\mathrm{A}}} \mathrm{H}_{\mathrm{B}} \mathrm{Ph}$ ), 3.66 (ddd, $J=1.5 \mathrm{~Hz}, J=4.5 \mathrm{~Hz}$, $\left.J=14.6 \mathrm{~Hz}, 1 \mathrm{H}, \mathrm{NCH}_{\mathrm{A}} \underline{\mathrm{H}}_{\mathrm{B}} \mathrm{CH}\right), 3.96\left(\mathrm{~d}, J=10.7 \mathrm{~Hz}, 3 \mathrm{H}, \mathrm{OCH}_{3}\right), 4.23$ (d, $J=14.2 \mathrm{~Hz}, 1 \mathrm{H}, \mathrm{NCH}_{\mathrm{A}} \underline{\mathrm{H}}_{\mathrm{B}} \mathrm{Ph}$ ), 5.03 (d, $\left.J=24.5 \mathrm{~Hz}, 1 \mathrm{H}, \mathrm{CHP}\right), 5.09$ (d, $J=10.8 \mathrm{~Hz}, 1 \mathrm{H}, \mathrm{HC}=\underline{\mathrm{C}}_{\mathrm{A}} \mathrm{H}_{\mathrm{B}}$ ), $5.18\left(\mathrm{dd}, J=1.5 \mathrm{~Hz}, J=17.3 \mathrm{~Hz}, 1 \mathrm{H}, \mathrm{HC}=\mathrm{CH}_{\mathrm{A}} \underline{H}_{\mathrm{B}}\right.$ ), 5.83 (dddd, $\left.J=4.5 \mathrm{~Hz}, J=7.2 \mathrm{~Hz}, J=10.5 \mathrm{~Hz}, J=17.3 \mathrm{~Hz}, 1 \mathrm{H}, \underline{\mathrm{HC}}=\mathrm{CH}_{2}\right), 7.19-7.44(\mathrm{~m}, 7 \mathrm{H}, 7 \mathrm{x}$ $\left.\mathrm{CH}_{\text {arom }}\right), 7.56$ (d, $\left.J=7.7 \mathrm{~Hz}, 1 \mathrm{H}, \mathrm{PCHCC} \underline{\mathrm{H}}\right), 7.97$ (d, $J=8.0 \mathrm{~Hz}, 1 \mathrm{H}$, PCHCCC $\left.\underline{\mathrm{H}}\right) .{ }^{13} \mathbf{C}-\mathbf{N M R}$ $\left(75 \mathbf{M H z}, \mathbf{C D C l}_{3}\right): \delta 52.94\left(\mathrm{~d}, J=6.9 \mathrm{~Hz}, \mathrm{OCH}_{3}\right), 53.72$ (d, $\left.J=6.9 \mathrm{~Hz}, \mathrm{OCH}_{3}\right), 54.28$ (d, $J=$ 
$8.1 \mathrm{~Hz}, \mathrm{NCH}_{2} \mathrm{CH}$ ), 55.18 (d, $J=8.1 \mathrm{~Hz}, \mathrm{NCH}_{2} \mathrm{Ph}$ ), 58.64 (d, $J=161.5 \mathrm{~Hz}, \mathrm{CHP}$ ), 81.98 $(\underline{\mathrm{CCH}}), 82.08(\mathrm{C} \underline{\mathrm{CH}}), 117.41\left(\mathrm{HC}=\underline{\mathrm{CH}}_{2}\right), 124.18(\mathrm{~d}, J=12.7 \mathrm{~Hz}, \mathrm{PCHC} \underline{\mathrm{C}}), 126.80\left(\mathrm{CH}_{\text {para }}\right.$, $\mathrm{Ph}), 128.11$ ( $3 \mathrm{x} \mathrm{CH}_{\text {arom }}$ ), 128.64 (3 x CHarom), 130.74 (d, $J=3.5 \mathrm{~Hz}$, PCHCCCH), 133.39 (PCHCCC $\mathrm{P}), 135.77$ (d, $J=5.8 \mathrm{~Hz}, \mathrm{PCHC}), 135.99\left(\mathrm{HC}=\mathrm{CH}_{2}\right), 139.96\left(\mathrm{C}_{\mathrm{q}}, \mathrm{Ph}\right) .{ }^{31} \mathbf{P}-\mathbf{N M R}$

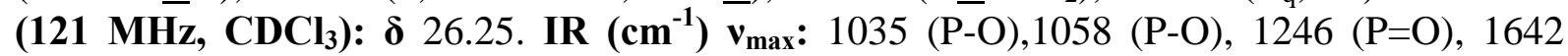
(C=C), 2099 (alkyne). MS (ESI): m/z (\%): $370.2\left(\mathrm{M}+\mathrm{H}^{+}, 100\right)$. MP $\left({ }^{\circ} \mathbf{C}\right):$ 86-87. Chromatography: Hex/EtOAc 4/6 $\mathrm{R}_{\mathrm{f}}=0.27$. Yield: $59 \%$.

\section{Dimethyl (2-ethynylphenyl) [(propyl)[(2E)-3-(4-methoxyphenyl)prop-2-enyl]amino]} methylphosphonate 2c

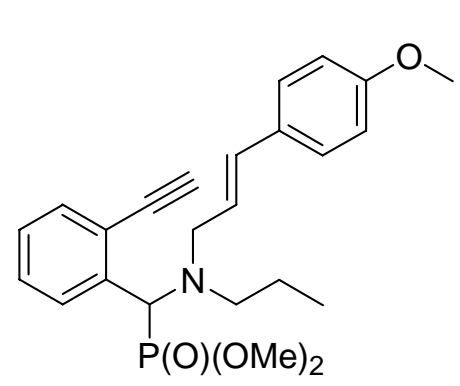

${ }^{1}$ H-NMR (300 MHz, $\left.\mathbf{C D C l}_{3}\right): \boldsymbol{\delta} 0.86\left(\mathrm{t}, J=7.3 \mathrm{~Hz}, 3 \mathrm{H}, \mathrm{CH}_{3}\right)$, 1.55 (sextet, $J=7.3 \mathrm{~Hz}, 2 \mathrm{H}, \mathrm{C}_{2} \mathrm{CH}_{3}$ ), 2.51 (ddd, $J=5.5 \mathrm{~Hz}, J=$ $\left.7.3 \mathrm{~Hz}, J=12.9 \mathrm{~Hz}, 1 \mathrm{H}, \mathrm{NC}_{\mathrm{H}_{\mathrm{A}}} \mathrm{H}_{\mathrm{B}} \mathrm{CH}_{2}\right), 2.74-2.81(\mathrm{~m}, 1 \mathrm{H}$, $\mathrm{NCH}_{\mathrm{A}} \underline{\mathrm{H}}_{\mathrm{B}} \mathrm{CH}_{2}$ ), 3.07 (dd, $J=7.6 \mathrm{~Hz}, J=14.3 \mathrm{~Hz}, 1 \mathrm{H}$, $\left.\mathrm{NCH}_{\mathrm{A}} \mathrm{H}_{\mathrm{B}} \mathrm{CH}\right), 3.22$ (s, $\left.1 \mathrm{H}, \mathrm{CCH}\right), 3.47(\mathrm{~d}, J=9.6 \mathrm{~Hz}, 3 \mathrm{H}$, $\left.\mathrm{OCH}_{3}\right), 3.79$ (s, 3H, $\left.\mathrm{PhOCH}_{3}\right), 3.90-3.94\left(\mathrm{~m}, 1 \mathrm{H}, \mathrm{NCH}_{\mathrm{A}} \mathrm{H}_{\mathrm{B}} \mathrm{CH}\right)$, 3.92 (d, $J=10.5 \mathrm{~Hz}, 3 \mathrm{H}, \mathrm{OCH}_{3}$ ), 5.02 (d, $\left.J=24.8 \mathrm{~Hz}, 1 \mathrm{H}, \mathrm{CHP}\right)$, 6.04 (ddd, $J=14.7 \mathrm{~Hz}, J=7.6 \mathrm{~Hz}, J=5.5 \mathrm{~Hz}, 1 \mathrm{H}, \underline{\mathrm{HC}}=\mathrm{CHPh}$ ), 6.41 (d, $J=14.7 \mathrm{~Hz}, 1 \mathrm{H}, \underline{\mathrm{HCPh}}$ ), 6.83 (d, $J=8.2 \mathrm{~Hz}, 2 \mathrm{H}, 2 \times \mathrm{CH}_{\text {arom}}$ ), 7.25-7.41 (m, 2H, 2 x $\left.\mathrm{CH}_{\text {arom }}\right), 7.26$ (d, $\left.J=8.3 \mathrm{~Hz}, 2 \mathrm{H}, 2 \times \mathrm{CH}_{\text {arom }}\right), 7.56$ (d, $\left.J=7.4 \mathrm{~Hz}, 1 \mathrm{H}, \mathrm{PCHCC} \underline{\mathrm{H}}\right), 7.93$ (d, $J=$ $7.7 \mathrm{~Hz}, 1 \mathrm{H}, \mathrm{PCHCCC} \underline{\mathrm{H}}) .{ }^{13} \mathbf{C}-\mathbf{N M R}\left(\mathbf{7 5} \mathbf{M H z}, \mathbf{C D C l}_{3}\right): \boldsymbol{\delta} 11.74\left(\mathrm{CH}_{2} \underline{\mathrm{CH}}{ }_{3}\right), 21.31\left(\underline{\mathrm{CH}}_{2} \mathrm{CH}_{3}\right)$, 52.76 (d, $J=6.9 \mathrm{~Hz}, \mathrm{OCH}_{3}$ ), 53.07 (d, $J=10.4 \mathrm{~Hz}, \mathrm{NCH}_{2} \mathrm{CH}_{2}$ ), 54.10 (d, $J=6.9 \mathrm{~Hz}, \mathrm{OCH}_{3}$ ), 54.20 (d, $J=5.8 \mathrm{~Hz}, \mathrm{NCH}_{2} \mathrm{CH}$ ), 58.95 (d, $\left.J=162.7 \mathrm{~Hz}, \mathrm{CHP}\right), 81.54(\mathrm{C} \underline{\mathrm{CH}}), 82.38$ (대), 114.00 (2 x CHarom), 124.01 (d, $J=12.7 \mathrm{~Hz}, \mathrm{PCHC} \underline{)}), 126.32$ (HC=CHPh), 127.42 (2 x $\left.\mathrm{CH}_{\text {arom }}\right), 127.91\left(\mathrm{CH}_{\text {arom }}\right), 128.66\left(\mathrm{CH}_{\text {arom }}\right), 130.32$ ( $\left.\mathrm{C}_{\text {q,arom }}\right), 130.73$ (d, $\left.J=3.5 \mathrm{~Hz}, \mathrm{PCHCC} H\right)$, $131.30(\mathrm{HC}=\underline{\mathrm{CHPh}}), 133.39(\mathrm{PCHCCCH}), 136.12(\mathrm{~d}, J=6.9 \mathrm{~Hz}, \mathrm{PCHC}), 159.01\left(\mathrm{C}_{\mathrm{q}}, \mathrm{Ph}\right)$. ${ }^{31}$ P-NMR (121 MHz, CDCl $)$ ): $\delta$ 26.11. IR (cm $\left.{ }^{-1}\right)$ v $_{\text {max }}$ : 1034 (P-O),1058 (P-O), $1248(\mathrm{P}=\mathrm{O})$, 1607 (C=C), 2098 (alkyne). MS (ESI): m/z (\%): $428.3\left(\mathrm{M}+\mathrm{H}^{+}, 100\right)$. MP $\left({ }^{\circ} \mathbf{C}\right): 99-101$. Yield: $81 \%$.

Dimethyl [(4-methylbenzyl)-(3-methylbut-2-enyl)amino] (2-ethynylphenyl) methylphosphonate $2 d$

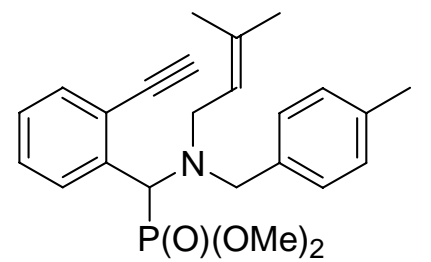

${ }^{1}$ H-NMR (300 MHz, $\left.\mathbf{C D C l}_{3}\right)$ : $\boldsymbol{\delta} 1.41\left(\mathrm{~s}, 3 \mathrm{H}, \mathrm{CH}_{3}\right), 1.65$ (s, 3H, $\mathrm{CH}_{3}$ ), 2.32 (s, 3H, $\mathrm{CH}_{3}$ ), 3.03 (dd, $J=6.7 \mathrm{~Hz}, J=13.5 \mathrm{~Hz}, 1 \mathrm{H}$, $\left.\mathrm{NC}_{\mathrm{H}} \mathrm{H}_{\mathrm{B}} \mathrm{CH}\right), 3.06$ (s, $\left.1 \mathrm{H}, \mathrm{CCH}\right), 3.40-3.51\left(\mathrm{~m}, 1 \mathrm{H}, \mathrm{NCH}_{\mathrm{A}} \underline{\mathrm{H}}_{\mathrm{B}} \mathrm{CH}\right)$, $3.44\left(\mathrm{~d}, J=10.4 \mathrm{~Hz}, 3 \mathrm{H}, \mathrm{OCH}_{3}\right), 3.53(\mathrm{~d}, J=13.8 \mathrm{~Hz}, 1 \mathrm{H}$, $\mathrm{NC}_{\mathrm{H}} \mathrm{H}_{\mathrm{B}} \mathrm{Ph}$ ), 3.89 (d, $\left.J=10.5 \mathrm{~Hz}, 3 \mathrm{H}, \mathrm{OCH}_{3}\right), 4.15$ (d, $J=13.8 \mathrm{~Hz}$, $1 \mathrm{H}, \mathrm{NCH}_{\mathrm{A}} \underline{\mathrm{H}}_{\mathrm{B}} \mathrm{Ph}$ ), 5.04 (d, $J=24.2 \mathrm{~Hz}, 1 \mathrm{H}, \mathrm{CHP}$ ), 5.26 (t*septet, $J$ $=6.6 \mathrm{~Hz}, J=1.2 \mathrm{~Hz}, 1 \mathrm{H}, \underline{\mathrm{HC}}=\mathrm{C}), 7.06-7.43\left(\mathrm{~m}, 6 \mathrm{H}, 6 \times \mathrm{CH}_{\text {arom }}\right), 7.55$ (d, $J=7.7 \mathrm{~Hz}, 1 \mathrm{H}$, PCHCC $\underline{H}), 7.96(\mathrm{~d}, J=8.0 \mathrm{~Hz}, 1 \mathrm{H}, \mathrm{PCHCCC} \underline{\mathrm{H}}) .{ }^{13} \mathrm{C}-\mathrm{NMR}\left(\mathbf{7 5} \mathbf{M H z}, \mathbf{C D C l}_{3}\right): \delta 18.19$ $\left(\mathrm{CH}_{3}\right), 21.22\left(\mathrm{CH}_{3}\right), 26.06\left(\mathrm{CH}_{3}\right), 49.05$ (d, $\left.J=9.2 \mathrm{~Hz}, \mathrm{NCH}_{2} \mathrm{CH}\right), 52.87$ (d, $J=6.9 \mathrm{~Hz}$, $\mathrm{OCH}_{3}$ ), 53.75 (d, $\left.J=6.9 \mathrm{~Hz}, \mathrm{OCH}_{3}\right), 55.15$ (d, $J=8.1 \mathrm{~Hz}, \mathrm{NCH}_{2} \mathrm{Ph}$ ), 58.86 (d, $J=160.4 \mathrm{~Hz}$,

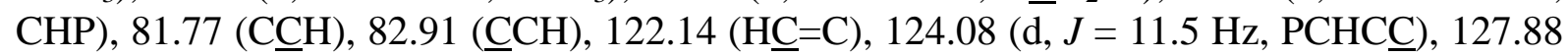
( $\left.\mathrm{CH}_{\text {arom }}\right), 128.63$ ( $\left.\mathrm{CH}_{\text {arom }}\right), 128.70$ (4 x CHarom), 130.79 (d, $J=3.5 \mathrm{~Hz}$, PCHCCGH), 133.33

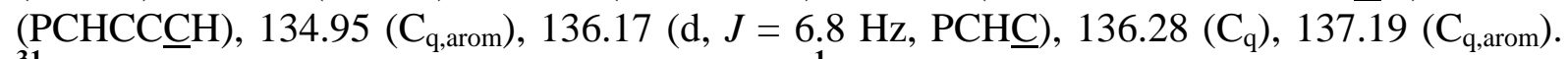
${ }^{31}$ P-NMR (121 MHz, CDCl $\left.{ }_{3}\right): \delta$ 26.56. IR (cm $\left.{ }^{-1}\right) \mathbf{v}_{\max }$ : 1035 (P-O), $1057(\mathrm{P}-\mathrm{O}), 1245(\mathrm{P}=\mathrm{O})$, 1637 (C=C), 2101 (alkyne). MS (ESI): m/z (\%): $412.3\left(\mathrm{M}+\mathrm{H}^{+}, 100\right)$. Chromatography: Hex/EtOAc $1 / 1 \mathrm{R}_{\mathrm{f}}=0.32$. MP $\left({ }^{\circ} \mathbf{C}\right):$ 101.5. Yield: $48 \%$. 
Dimethyl (2-ethynylphenyl)[[(2E)-4-methylpent-2-enyl(2-phenylethyl)]amino] methylphosphonate $2 \mathrm{e}$<smiles>C#Cc1ccccc1C(C(=O)OC)N(CC=CC(C)C)CCc1ccccc1</smiles>
1H, PCHCC $\underline{H}$ ), 7.89 (d, $J=7.7 \mathrm{~Hz}, 1 \mathrm{H}$, PCHCCC브). ${ }^{13} \mathbf{C}-\mathbf{N M R}\left(\mathbf{7 5} \mathbf{~ M H z}, \mathbf{C D C l}_{3}\right): \delta 22.51$ $\left(\mathrm{CH}_{3}\right), 22.56\left(\mathrm{CH}_{3}\right), 30.99(\mathrm{CH}), 34.23\left(\mathrm{CH}_{2} \mathrm{Ph}\right), 52.49$ (d, $\left.J=10.4 \mathrm{~Hz}, \mathrm{NCH}_{2} \mathrm{CH}_{2}\right), 52.80$ (d, $\left.J=8.1 \mathrm{~Hz}, \mathrm{OCH}_{3}\right), 54.16\left(\mathrm{~d}, J=5.8 \mathrm{~Hz}, \mathrm{NCH}_{2} \mathrm{CH}+\mathrm{OCH}_{3}\right), 59.51(\mathrm{~d}, J=163.8 \mathrm{~Hz}, \mathrm{CHP})$, $81.91(\mathrm{C} \underline{\mathrm{CH}}), 82.55(\underline{\mathrm{CCH}}), 123.81(\mathrm{~d}, J=12.7 \mathrm{~Hz}, \mathrm{PCHC} \underline{\mathrm{C}}), 124.78\left(\mathrm{NCH}_{2} \underline{\mathrm{CH}}\right), 125.89$ ( $\left.\mathrm{CH}_{\text {arom }}\right), 127.96$ ( $\left.\mathrm{CH}_{\text {arom }}\right), 128.26$ (2 x CH $\left.\mathrm{CH}_{\text {arom }}\right), 128.78$ ( $\left.\mathrm{CH}_{\text {arom }}\right), 129.18$ (2 x $\left.\mathrm{CH}_{\text {arom }}\right), 130.60$ (d, $J=3.5 \mathrm{~Hz}$, PCHCCGH), $133.44\left(\mathrm{CH}_{\mathrm{arom}}\right), 136.25$ (d, $\left.J=4.6 \mathrm{~Hz}, \mathrm{PCHC}\right), 140.54$ $\left(\mathrm{HCCH}\left(\mathrm{CH}_{3}\right)_{2}\right), 140.58\left(\mathrm{C}_{\mathrm{q}, \text { arom }}\right) .{ }^{31} \mathbf{P}-\mathbf{N M R}\left(\mathbf{M H z}, \mathbf{C D C l}_{\mathbf{3}}\right): \boldsymbol{\delta}$ 25.95. IR $\left(\mathbf{c m}^{\mathbf{- 1}}\right) \mathbf{v}_{\mathbf{m a x}}$ : $1035(\mathrm{P}-$ O), 1060 (P-O), 1246 ( $\mathrm{P}=\mathrm{O}), 1604$ (C=C), 2100 (alkyne). MS (ESI): m/z (\%): $426.2\left(\mathrm{M}+\mathrm{H}^{+}\right.$, 100). Chromatography: Hex/EtOAc $1 / 1 \mathrm{R}_{\mathrm{f}}=0.56$. Yield: $56 \%$.

\section{Dimethyl [allyl(3-fluorobenzyl)amino](2-ethynylphenyl)methylphosphonate $2 f$}

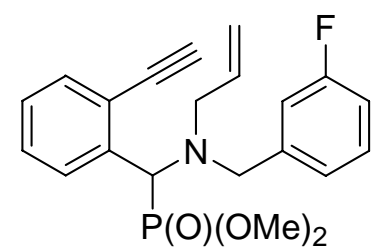

${ }^{1}$ H-NMR (300 MHz, $\mathbf{C D C l}_{3}$ ): $\boldsymbol{\delta} 3.05$ (dd, $J=7.6 \mathrm{~Hz}, J=14.3 \mathrm{~Hz}, 1 \mathrm{H}$, $\left.\mathrm{NCH}_{\mathrm{A}} \mathrm{H}_{\mathrm{B}} \mathrm{CH}\right), 3.06$ (s, $\left.1 \mathrm{H}, \mathrm{CCH}\right), 3.44$ (d, $J=10.7 \mathrm{~Hz}, 3 \mathrm{H}, \mathrm{OCH}_{3}$ ), $3.53\left(\mathrm{~d}, J=14.4 \mathrm{~Hz}, 1 \mathrm{H}, \mathrm{NCH}_{\mathrm{A}} \mathrm{H}_{\mathrm{B}} \mathrm{Ph}\right.$ ), 3.71 (ddd, $J=2.0 \mathrm{~Hz}, J=4.6$ $\left.\mathrm{Hz}, J=14.3 \mathrm{~Hz}, 1 \mathrm{H}, \mathrm{NCH}_{\mathrm{A}} \underline{\mathrm{H}}_{\mathrm{B}} \mathrm{CH}\right), 3.91\left(\mathrm{~d}, J=10.7 \mathrm{~Hz}, 3 \mathrm{H}, \mathrm{OCH}_{3}\right)$, $4.21\left(\mathrm{~d}, J=14.4 \mathrm{~Hz}, 1 \mathrm{H}, \mathrm{NCH}_{\mathrm{A}} \underline{\mathrm{H}}_{\mathrm{B}} \mathrm{Ph}\right), 4.99(\mathrm{~d}, J=24.8 \mathrm{~Hz}, 1 \mathrm{H}$, CHP), 5.10 (dd, $J=10.6 \mathrm{~Hz}, J=1.0 \mathrm{~Hz}, 1 \mathrm{H}, \mathrm{HC}=\mathrm{CH}_{\mathrm{A}} \mathrm{H}_{\mathrm{B}}$ ), 5.18 (dd, $J=1.0 \mathrm{~Hz}, J=17.0 \mathrm{~Hz}$, $1 \mathrm{H}, \mathrm{HC}=\mathrm{CH}_{\mathrm{A}} \underline{\mathrm{H}}_{\mathrm{B}}$ ), 5.83 (dddd, $J=4.6 \mathrm{~Hz}, J=7.6 \mathrm{~Hz}, J=10.6 \mathrm{~Hz}, J=17.0 \mathrm{~Hz}, 1 \mathrm{H}$, $\left.\underline{\mathrm{HC}}=\mathrm{CH}_{2}\right), 6.87-7.44\left(\mathrm{~m}, 6 \mathrm{H}, 6 \times \mathrm{CH}_{\text {arom }}\right), 7.56(\mathrm{~d}, J=7.7 \mathrm{~Hz}, 1 \mathrm{H}, \mathrm{PCHCC} \underline{\mathrm{H}}), 7.96$ (d, $J=7.7$ $\mathrm{Hz}, 1 \mathrm{H}, \mathrm{PCHCCCH}) .{ }^{13} \mathbf{C}-\mathbf{N M R}\left(\mathbf{7 5} \mathbf{M H z}, \mathbf{C D C l}_{3}\right): \boldsymbol{\delta} 53.00\left(\mathrm{~d}, J=6.9 \mathrm{~Hz}, \mathrm{OCH}_{3}\right), 53.62$ (d, $\left.J=6.9 \mathrm{~Hz}, \mathrm{OCH}_{3}\right), 54.47$ (d, $\left.J=6.9 \mathrm{~Hz}, \mathrm{NCH}_{2} \mathrm{CH}\right), 54.65$ (d, $\left.J=8.1 \mathrm{~Hz}, \mathrm{NCH}_{2} \mathrm{Ph}\right), 58.53$ (d, $J=161.5 \mathrm{~Hz}, \mathrm{CHP}), 81.91$ (대), $82.11(\underline{\mathrm{CCH}}), 113.62$ (d, $\left.J=20.8 \mathrm{~Hz}, \mathrm{CH}_{\text {arom}}\right), 115.26$ (d, $J$ $\left.=21.9 \mathrm{~Hz}, \mathrm{CH}_{\mathrm{arom}}\right), 117.60\left(\mathrm{HC}=\underline{\mathrm{CH}}_{2}\right), 124.08$ (d, $\left.J=2.3 \mathrm{~Hz}, \mathrm{CH}_{\text {arom }}\right), 124.18(\mathrm{~d}, J=12.7 \mathrm{~Hz}$, PCHCC), 128.17 ( $\left.\mathrm{CH}_{\text {arom }}\right), 128.75$ ( $\left.\mathrm{CH}_{\text {arom }}\right), 129.38$ (d, $\left.J=8.1 \mathrm{~Hz}, \mathrm{CH}_{\text {arom }}\right), 130.64$ (d, $J=3.5$ $\mathrm{Hz}, \mathrm{PCHCCH}), 133.45$ (PCHCCCCH), 135.48 (d, J = $5.8 \mathrm{~Hz}, \mathrm{PCHC}), 135.79\left(\mathrm{HC}=\mathrm{CH}_{2}\right)$, 142.90 (d, $\left.J=6.9 \mathrm{~Hz}, \mathrm{C}_{\mathrm{q}}, \mathrm{Ph}\right), 163.03$ (d, $J=224.6 \mathrm{~Hz}, \mathrm{FC}_{\mathrm{q}}$,arom). ${ }^{31}$ P-NMR (121 MHz, $\left.\mathbf{C D C l}_{3}\right): \boldsymbol{\delta} 26.03 .{ }^{19} \mathbf{F}-\mathbf{N M R}\left(\mathbf{2 8 2} \mathbf{~ M H z}, \mathbf{C D C l}_{3}\right): \boldsymbol{\delta}-113.90(\mathrm{dt}, J=6.6 \mathrm{~Hz}, J=9.2 \mathrm{~Hz}) . \mathbf{I R}$ $\left(\mathbf{c m}^{-1}\right) v_{\text {max }}: 1035$ (P-O), 1058 (P-O), $1248(\mathrm{P}=\mathrm{O}), 1615(\mathrm{C}=\mathrm{C}), 2100$ (alkyne). MS (ESI): m/z (\%): $388.3\left(\mathrm{M}+\mathrm{H}^{+}, 100\right)$. Chromatography: Hex/EtOAc 6/4 $\mathrm{R}_{\mathrm{f}}=0.13$. Yield: $72 \%$. 
Dimethyl [[(cyclohex-1-en-1-ylmethyl)[2-(4-chlorophenyl)ethyl]]amino] (2ethynylphenyl)methylphosphonate $2 \mathrm{~g}$

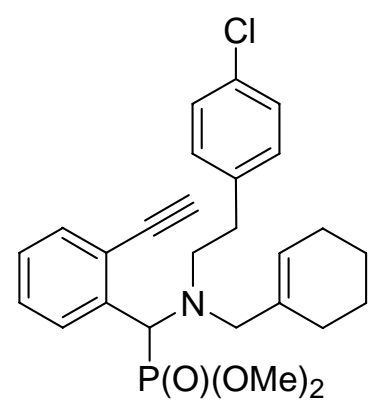

${ }^{1}$ H-NMR (300 MHz, $\left.\mathbf{C D C l}_{3}\right)$ : $\boldsymbol{\delta}$ 1.53-1.61 (m, 4H, $\left.\mathrm{CH}_{2} \mathrm{CH}_{2}\right)$, 1.86$1.97\left(\mathrm{~m}, 4 \mathrm{H}, \mathrm{HCC} \underline{H}_{2}+\mathrm{CC}_{2}\right)$, 2.57-2.68 (m, 1H, $\left.\mathrm{NC}_{\mathrm{H}} \mathrm{H}_{\mathrm{B}} \mathrm{CH}_{2}\right), 2.74$ (t, $\left.J=6.6 \mathrm{~Hz}, 2 \mathrm{H}, \mathrm{NCH}_{2} \underline{\mathrm{C}}_{2}\right), 2.84\left(\mathrm{~d}, J=12.9 \mathrm{~Hz}, \overline{\mathrm{NC}}_{\mathrm{A}} \mathrm{H}_{\mathrm{B}}\right), 2.96-$ $3.05\left(\mathrm{~m}, 1 \mathrm{H}, \mathrm{NCH}_{\mathrm{A}} \underline{\mathrm{H}}_{\mathrm{B}} \mathrm{CH}_{2}\right.$ ), 3.31 (s, $1 \mathrm{H}, \mathrm{CH}_{\text {alkyne }}$ ), 3.44 (d, $J=10.5$ $\left.\mathrm{Hz}, 3 \mathrm{H}, \mathrm{OCH}_{3}\right), 3.65$ (d, $\left.J=12.9 \mathrm{~Hz}, \mathrm{NCH}_{\mathrm{A}} \underline{\mathrm{H}}_{\mathrm{B}}\right), 3.82$ (d, $J=10.7 \mathrm{~Hz}$, $3 \mathrm{H}, \mathrm{OCH}_{3}$ ), 5.03 (d, $J=24.8 \mathrm{~Hz}, 1 \mathrm{H}, \mathrm{CHP}$ ), 5.52 (br s, $1 \mathrm{H}, \mathrm{CH}$ ), 7.09 (d, $\left.J=8.4 \mathrm{~Hz}, 2 \mathrm{H}, 2 \times \mathrm{CH}_{\text {arom }}\right), 7.21$ (d, $J=8.4 \mathrm{~Hz}, 2 \mathrm{H}, 2 \times \mathrm{CH}_{\text {arom }}$ ), 7.29-7.39 (m, 2H, $2 \times \mathrm{CH}_{\text {arom }}$ ), 7.57 (d, $J=7.4 \mathrm{~Hz}, 1 \mathrm{H}, \mathrm{PCHCC} \underline{\mathrm{H}}$ ), 7.85 (d, $J=8.0 \mathrm{~Hz}, 1 \mathrm{H}, \mathrm{PCHCCC} \underline{\mathrm{H}}) .{ }^{13} \mathbf{C}-\mathbf{N M R}\left(\mathbf{7 5} \mathbf{M H z}, \mathbf{C D C l}_{3}\right): \boldsymbol{\delta}$ $22.73\left(\mathrm{CH}_{2}\right), 22.91\left(\mathrm{CH}_{2}\right), 25.38\left(\mathrm{CH}_{2}\right), 26.77\left(\mathrm{CH}_{2}\right), 33.74\left(\mathrm{CH}_{2} \mathrm{Ph}\right), 52.58(\mathrm{~d}, J=10.4 \mathrm{~Hz}$, $\mathrm{NCH}_{2} \mathrm{CH}_{2}$ ), 52.91 (d, $\left.J=6.9 \mathrm{~Hz}, \mathrm{OCH}_{3}\right), 53.59$ (d, $\left.J=6.9 \mathrm{~Hz}, \mathrm{OCH}_{3}\right), 59.00$ (d, $J=162.7$ $\mathrm{Hz}, \mathrm{CHP}), 59.26$ (d, $\left.J=5.8 \mathrm{~Hz}, \mathrm{NC}_{2} \mathrm{C}\right), 81.71(\mathrm{C} \underline{\mathrm{CH}}), 82.55(\underline{\mathrm{CCH}}), 123.87$ (d, $J=12.7 \mathrm{~Hz}$, PCHCC $), 124.72(\mathrm{CH}), 127.96\left(\mathrm{CH}_{\text {arom }}\right), 128.32$ (2 x CHarom $), 128.73\left(\mathrm{CH}_{\text {arom }}\right), 130.37$ (2 x $\left.\mathrm{CH}_{\text {arom }}\right), 130.64$ (d, $\left.J=3.5 \mathrm{~Hz}, \mathrm{PCHCCH}\right), 131.57\left(\mathrm{ClC}_{\mathrm{q}, \text { arom }}\right), 133.44\left(\mathrm{CH}_{\text {arom }}\right), 135.96$ $\left(\mathrm{C}_{\mathrm{q}, \text { arom }}\right), 136.06$ (d, $\left.J=5.8 \mathrm{~Hz}, \mathrm{PCH} \underline{\mathrm{C}}\right), 139.24(\underline{\mathrm{C}}=\mathrm{CH}) .{ }^{31} \mathbf{P}-\mathbf{N M R}\left(\mathbf{M H z}, \mathbf{C D C l}_{\mathbf{3}}\right): \boldsymbol{\delta} 26.21$. IR $\left(\mathbf{c m}^{-1}\right) \mathbf{v}_{\text {max }}$ : 1035 (P-O), 1060 (P-O), $1244(\overline{\mathrm{P}}=\mathrm{O}), 2097$ (alkyne). MS (ESI): m/z (\%): 472.2/474.2 $\left(\mathrm{M}+\mathrm{H}^{+}, 100\right)$. MP $\left({ }^{\circ} \mathrm{C}\right): 103-104$. Yield: $59 \%$.

\section{Dimethyl (2-ethynylphenyl)\{butyl[(2E)-2-methyl-3-phenylprop-2-enyl]amino\} methylphosphonate $2 \mathrm{~h}$}

For purification of $\mathbf{2} \mathbf{h}$ : The mixture obtained after drying with $\mathrm{MgSO}_{4}$ was dissolved in ether $(100 \mathrm{~mL})$ and washed twice with $\mathrm{HCl}(3 \mathrm{~N}, 25 \mathrm{~mL})$ to remove the excess of secondary amine. Afterwards the organic layer is made basic with $\mathrm{NaOH}(\mathrm{aq}, 3 \mathrm{~N})$, extracted with ether three times (50 mL) and dried with $\mathrm{MgSO}_{4}$.

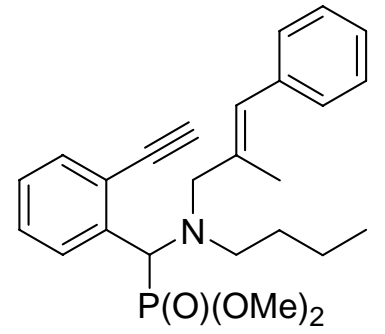

${ }^{1} \mathbf{H}-\mathbf{N M R}\left(300 \mathrm{MHz}, \mathbf{C D C l}_{3}\right): \boldsymbol{\delta} 0.89\left(\mathrm{t}, J=7.3 \mathrm{~Hz}, 3 \mathrm{H}, \mathrm{CH}_{2} \mathrm{CH}_{3}\right)$, 1.20-1.43 (m, $\left.2 \mathrm{H}, \mathrm{C}_{2} \mathrm{CH}_{3}\right), 1.54\left(\mathrm{~m}, 2 \mathrm{H}, \mathrm{NCH}_{2} \underline{\mathrm{C}}_{2}\right), 1.92$ (s, $3 \mathrm{H}$, $\mathrm{CCH}_{3}$ ), 2.44 (ddd, $J=6.1 \mathrm{~Hz}, J=6.9 \mathrm{~Hz}, J=12.9 \mathrm{~Hz}, 1 \mathrm{H}$, $\left.\mathrm{NC}_{\mathrm{H}_{\mathrm{A}}} \mathrm{H}_{\mathrm{B}} \mathrm{CH}_{2}\right), 2.81-2.92\left(\mathrm{~m}, 1 \mathrm{H}, \mathrm{NCH}_{\mathrm{A}} \underline{\mathrm{H}}_{\mathrm{B}} \mathrm{CH}_{2}\right), 2.90$ (d, $J=13.8 \mathrm{~Hz}$, $1 \mathrm{H}, \mathrm{NCH}_{\mathrm{A}} \mathrm{H}_{\mathrm{B}} \mathrm{C}$ ), 3.21 (s, $\left.1 \mathrm{H}, \mathrm{CCH}\right), 3.46$ (d, $J=10.5 \mathrm{~Hz}, 3 \mathrm{H}, \mathrm{OCH}_{3}$ ), $3.84\left(\mathrm{~d}, J=13.8 \mathrm{~Hz}, 1 \mathrm{H}, \mathrm{NCH}_{\mathrm{A}} \underline{\mathrm{H}}_{\mathrm{B}} \mathrm{C}\right), 3.91(\mathrm{~d}, J=10.7 \mathrm{~Hz}, 3 \mathrm{H}$, $\mathrm{OCH}_{3}$ ), 5.05 (d, $J=25.6 \mathrm{~Hz}, 1 \mathrm{H}, \mathrm{CHP}$ ), 6.43 (s, 1H, HC=C), 7.17-7.43 (m, 7H, $\left.7 \times \mathrm{CH}_{\text {arom }}\right), 7.57$ (d, $\left.J=7.4 \mathrm{~Hz}, 1 \mathrm{H}, \mathrm{PCHCC} \underline{\mathrm{H}}\right), 7.93(\mathrm{~d}, J=8.0 \mathrm{~Hz}, 1 \mathrm{H}$, PCHCCCH $) .{ }^{13} \mathbf{C}-\mathbf{N M R}\left(75 \mathbf{M H z}, \mathbf{C D C l}_{3}\right): \delta 14.27\left(\mathrm{CH}_{2} \underline{\mathrm{CH}}_{3}\right), 16.65\left(\mathrm{CCCH}_{3}\right), 20.56$

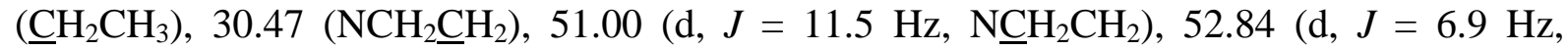
$\mathrm{OCH}_{3}$ ), 53.63 (d, $\left.J=6.9 \mathrm{~Hz}, \mathrm{OCH}_{3}\right), 58.30$ (d, $\left.J=163.8 \mathrm{~Hz}, \mathrm{CHP}\right), 60.97$ (d, $J=5.8 \mathrm{~Hz}$,

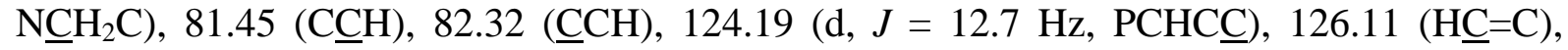
126.81 ( $\left.\mathrm{CH}_{\text {arom }}\right), 127.94$ ( $\left.\mathrm{CH}_{\text {arom }}\right), 128.12$ (2 x CHarom $), 128.63$ ( $\left.\mathrm{CH}_{\text {arom }}\right), 128.93$ (2 x $\left.\mathrm{CH}_{\text {arom }}\right)$, 130.80 (d, $J=3.5 \mathrm{~Hz}, \mathrm{PCHCC} H), 133.42(\mathrm{PCHCCCH}), 135.76$ (d, $J=6.9 \mathrm{~Hz}, \mathrm{PCHC})$, $137.35(\underline{C}=\mathrm{CH}), 138.45\left(\mathrm{C}_{\mathrm{q}}, \mathrm{Ph}\right) .{ }^{31} \mathbf{P}-\mathbf{N M R}\left(\mathbf{1 2 1} \mathbf{M H z}, \mathbf{C D C l}_{\mathbf{3}}\right): \boldsymbol{\delta}$ 26.38. IR $\left(\mathbf{c m}^{-1}\right) \mathbf{v}_{\max }$ : 1036 (P-O),1059 (P-O), 1244 (P=O), 1598 (C=C), 2099 (alkyne). MS (ESI): m/z (\%): 426.2 $\left(\mathrm{M}+\mathrm{H}^{+}, 100\right)$. Yield: $88 \%$. 


\section{Dimethyl (diallylamino)(2-ethynylphenyl)methylphosphonate 2i}

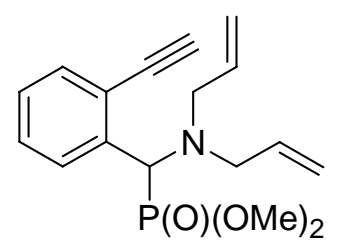

${ }^{1}$ H-NMR (300 MHz, $\left.\mathbf{C D C l}_{3}\right)$ : $\boldsymbol{\delta} 3.06$ (dd, $J=7.2 \mathrm{~Hz}, J=14.6 \mathrm{~Hz}, 2 \mathrm{H}, 2$ x NC$\left.\underline{\mathrm{N}}_{\mathrm{A}} \mathrm{H}_{\mathrm{B}} \mathrm{CH}\right), 3.26$ (s, $\left.1 \mathrm{H}, \mathrm{CCH}\right), 3.46\left(\mathrm{~d}, J=10.5 \mathrm{~Hz}, 3 \mathrm{H}, \mathrm{OCH}_{3}\right)$, 3.66 (ddt, $J=2.2 \mathrm{~Hz}, J=2.5 \mathrm{~Hz}, J=14.6 \mathrm{~Hz}, 2 \mathrm{H}, 2 \times \mathrm{NCH}_{\mathrm{A}} \underline{\mathrm{H}}_{\mathrm{B}} \mathrm{CH}$ ), 3.90 (d, $J=10.5 \mathrm{~Hz}, 3 \mathrm{H}, \mathrm{OCH}_{3}$ ), 4.97 (d, $\left.J=24.2 \mathrm{~Hz}, 1 \mathrm{H}, \mathrm{CHP}\right), 5.10$ (d, $J=$ $\left.10.5 \mathrm{~Hz}, 2 \mathrm{H}, 2 \times \mathrm{HC}=\mathrm{C}_{\mathrm{H}} \mathrm{H}_{\mathrm{b}}\right), 5.19\left(\mathrm{~d}, J=17.2 \mathrm{~Hz}, 2 \mathrm{H}, 2 \mathrm{x} \mathrm{HC}=\mathrm{CH}_{\mathrm{a}} \underline{\mathrm{H}}_{\mathrm{b}}\right)$, 5.75-5.88 (m, 2H, 2 x $\left.\underline{\mathrm{HC}}=\mathrm{CH}_{2}\right), 7.27-7.41$ (m, 2H, $\left.2 \times \mathrm{CH}_{\text {arom }}\right), 7.72$ (d, $J=7.5 \mathrm{~Hz}, 1 \mathrm{H}$, PCHCC $\underline{\mathrm{H}}), 7.92(\mathrm{~d}, J=7.7 \mathrm{~Hz}, 1 \mathrm{H}$, PCHCCC $\underline{\mathrm{H}}) .{ }^{13} \mathbf{C}-\mathbf{N M R}(\mathbf{7 5} \mathbf{~ M H z}$, $\mathbf{C D C l}_{3}$ ): $\boldsymbol{\delta} 52.82$ (d, $\left.J=6.9 \mathrm{~Hz}, \mathrm{OCH}_{3}\right), 54.05$ (d, $J=8.1 \mathrm{~Hz}, 2$ x NCH $\mathrm{N}_{2} \mathrm{CH}$ ), 54.15 (d, $J=6.9$ $\left.\mathrm{Hz}, \mathrm{OCH}_{3}\right), 58.91$ (d, $\left.J=163.8 \mathrm{~Hz}, \mathrm{CHP}\right), 81.97(\mathrm{CC} H), 82.26(\underline{\mathrm{CCH}}), 117.04\left(2 \times \mathrm{HC}=\underline{\mathrm{CH}}_{2}\right)$, 123.95 (d, $J=12.7 \mathrm{~Hz}, \mathrm{PCHC}$ ), $127.99\left(\mathrm{CH}_{\text {arom }}\right), 128.70\left(\mathrm{CH}_{\text {arom }}\right), 130.66$ (d, $J=4.6 \mathrm{~Hz}$, PCHCCH), $133.42\left(\mathrm{CH}_{\text {arom }}\right), 136.00$ (d, $\left.J=4.6 \mathrm{~Hz}, \mathrm{PCHC}\right), 136.15\left(2 \times \mathrm{HC}=\mathrm{CH}_{2}\right) .{ }^{31} \mathbf{P}-\mathbf{N M R}$

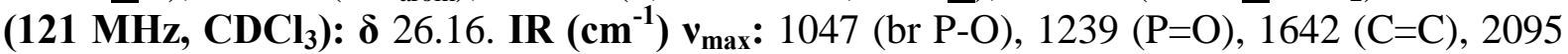
(alkyne). MS (ESI): m/z (\%): $320.2\left(\mathrm{M}+\mathrm{H}^{+}, 100\right)$. MP ( $\left.{ }^{\circ} \mathbf{C}\right): 97$. Yield: $79 \%$.

\section{Dimethyl (2-ethynylphenyl) [(butyl)(3-fluorobenzyl)amino]methylphosphonate 2j}

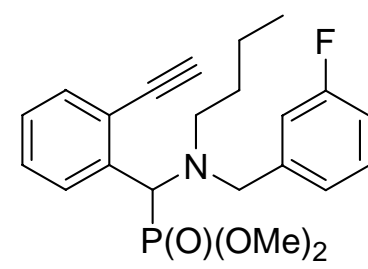

${ }^{1} \mathbf{H}-N M R\left(300 ~ M H z, \mathbf{C D C l}_{3}\right): \delta 0.81\left(\mathrm{t}, J=7.4 \mathrm{~Hz}, 3 \mathrm{H}, \mathrm{CH}_{3}\right), 1.09-$ 1.36 (m, 2H, $\left.\underline{\mathrm{C}}_{2} \mathrm{CH}_{3}\right), 1.48$ (p, $J=7.2 \mathrm{~Hz}, 2 \mathrm{H}, \mathrm{NCH}_{2} \mathrm{C}_{2}$ ), 2.45 (dt, $J$ $\left.=6.6 \mathrm{~Hz}, J=13.0 \mathrm{~Hz}, 1 \mathrm{H}, \mathrm{NC}_{\mathrm{H}_{\mathrm{A}}} \mathrm{H}_{\mathrm{B}} \mathrm{CH}_{2}\right), 2.70-2.80(\mathrm{~m}, 1 \mathrm{H}$, $\left.\mathrm{NCH}_{\mathrm{A}} \underline{\mathrm{H}}_{\mathrm{B}} \mathrm{CH}_{2}\right), 3.14(\mathrm{~s}, 1 \mathrm{H}, \mathrm{CCH}), 3.37$ (d, $J=14.3 \mathrm{~Hz}, 1 \mathrm{H}$, $\mathrm{NC}_{\mathrm{H}_{\mathrm{A}}} \mathrm{H}_{\mathrm{B}} \mathrm{Ph}$ ), 3.46 (d, $\left.J=10.5 \mathrm{~Hz}, 3 \mathrm{H}, \mathrm{OCH}_{3}\right), 3.92$ (d, $J=10.7 \mathrm{~Hz}$, $3 \mathrm{H}, \mathrm{OCH}_{3}$ ), 4.37 (d, $J=14.3 \mathrm{~Hz}, 1 \mathrm{H}, \mathrm{NCH}_{\mathrm{A}} \underline{\mathrm{H}}_{\mathrm{B}} \mathrm{Ph}$ ), 5.02 (d, $J=25.3$

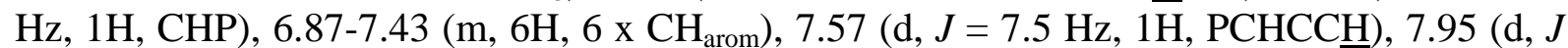

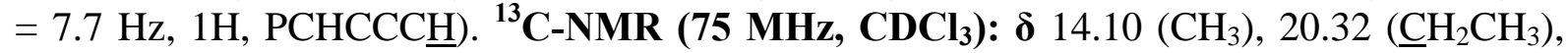
$30.32\left(\mathrm{NCH}_{2} \mathrm{CH}_{2}\right), 51.32$ (d, $\left.J=10.4 \mathrm{~Hz}, \mathrm{NCH}_{2} \mathrm{CH}_{2}\right), 52.92$ (d, $\left.J=8.1 \mathrm{~Hz}, \mathrm{OCH}_{3}\right), 53.63$ (d, $\left.J=6.9 \mathrm{~Hz}, \mathrm{OCH}_{3}\right), 55.61$ (d, $J=5.8 \mathrm{~Hz}, \mathrm{NCCH}_{2} \mathrm{Ph}$ ), 58.30 (d, $\left.J=163.8 \mathrm{~Hz}, \mathrm{CHP}\right), 81.62$ (Cㅡㅐ), $82.11(\underline{\mathrm{CCH}}), 113.56$ (d, $\left.J=20.8 \mathrm{~Hz}, \mathrm{CH}_{\text {arom }}\right), 115.37$ (d, $\left.J=21.9 \mathrm{~Hz}, \mathrm{CH}_{\text {arom}}\right)$, 124.17 (d, $J=2.3 \mathrm{~Hz}, \mathrm{CH}_{\text {arom }}$ ), 124.23 (d, $\left.J=11.5 \mathrm{~Hz}, \mathrm{PCHC} \underline{\mathrm{C}}\right), 128.09\left(\mathrm{CH}_{\text {arom}}\right), 128.70$ ( $\mathrm{CH}_{\text {arom }}$ ), 129.38 (d, $\left.J=8.1 \mathrm{~Hz}, \mathrm{CH}_{\text {arom }}\right), 130.71$ (d, $\left.J=3.5 \mathrm{~Hz}, \mathrm{PCHC} \underline{\mathrm{CH}}\right), 133.47$ (PCHCCCH), 135.47 (d, $J=6.9 \mathrm{~Hz}, \mathrm{PCHC}), 143.36$ (d, $\left.J=6.9 \mathrm{~Hz}, \mathrm{C}_{\mathrm{q}}, \mathrm{Ph}\right), 163.00$ (d, $J=$ $224.6 \mathrm{~Hz}, \mathrm{FC}_{\mathrm{q}}$,arom $) .{ }^{31} \mathbf{P}-\mathrm{NMR}\left(121 \mathrm{MHz}, \mathbf{C D C l}_{3}\right): \delta$ 26.37. ${ }^{19} \mathbf{F}-\mathrm{NMR}\left(\mathbf{2 8 2} \mathbf{~ M H z}, \mathbf{C D C l}_{3}\right): \delta$

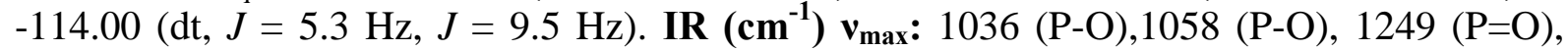
1614 (C=C), 2099 (alkyne). MS (ESI): m/z (\%): $404.2\left(\mathrm{M}+\mathrm{H}^{+}, 100\right)$. MP ( $\left.{ }^{\circ} \mathrm{C}\right): 67$. Yield: $65 \%$.

\section{Dimethyl (2-ethynylphenyl)[(4-methoxybenzyl)(propyl)amino]methylphosphonate 2k}

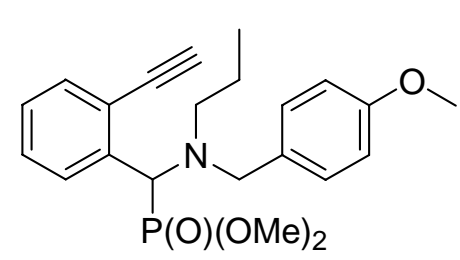

${ }^{1}$ H-NMR (300 MHz, $\left.\mathbf{C D C l}_{3}\right)$ : $\boldsymbol{\delta} 0.77\left(\mathrm{t}, J=7.3 \mathrm{~Hz}, 3 \mathrm{H}, \mathrm{CH}_{3}\right)$, 1.42-1.57 (m, 2H, $\mathrm{CH}_{2} \mathrm{CH}_{3}$ ), 2.41 (ddd, $J=4.8 \mathrm{~Hz}, J=8.3 \mathrm{~Hz}, J$ $\left.=13.0 \mathrm{~Hz}, 1 \mathrm{H}, \mathrm{NC}_{\mathrm{H}_{\mathrm{A}}} \mathrm{H}_{\mathrm{B}} \mathrm{CH}_{2}\right), 2.61-2.72\left(\mathrm{~m}, 1 \mathrm{H}, \mathrm{NCH}_{\mathrm{A}} \underline{\mathrm{H}}_{\mathrm{B}} \mathrm{CH}_{2}\right)$, 3.16 (s, 1H, CCH), 3.27 (d, $J=13.5 \mathrm{~Hz}, 1 \mathrm{H}, \mathrm{NC}_{\mathrm{H}_{\mathrm{A}}} \mathrm{H}_{\mathrm{B}} \mathrm{Ph}$ ), 3.47 (d, $\left.J=10.5 \mathrm{~Hz}, 3 \mathrm{H}, \mathrm{OCH}_{3}\right), 3.79$ (s, 3H, $\left.\mathrm{PhOCH}_{3}\right), 3.91$ (d, $J=$ $\left.10.7 \mathrm{~Hz}, 3 \mathrm{H}, \mathrm{OCH}_{3}\right), 4.32$ (d, $\left.J=13.5 \mathrm{~Hz}, 1 \mathrm{H}, \mathrm{NCH}_{\mathrm{A}} \underline{\mathrm{H}}_{\mathrm{B}} \mathrm{Ph}\right), 5.04$ (d, $J=25.3 \mathrm{~Hz}, 1 \mathrm{H}, \mathrm{CHP}), 6.83\left(\mathrm{~d}, J=8.7 \mathrm{~Hz}, 2 \mathrm{H}, 2 \times \mathrm{CH}_{\text {arom }}\right), 7.26-7.56(\mathrm{~m}, 2 \mathrm{H}, 2 \mathrm{x}$ $\mathrm{CH}_{\text {arom }}$ ), 7.28 (d, $\left.J=8.7 \mathrm{~Hz}, 2 \mathrm{H}, 2 \times \mathrm{CH}_{\text {arom }}\right), 7.57$ (d, $J=6.0 \mathrm{~Hz}, 1 \mathrm{H}$, PCHCC $\left.\underline{H}\right), 7.96$ (d, $J=$ $7.7 \mathrm{~Hz}, 1 \mathrm{H}$, PCHCCC프). ${ }^{13} \mathbf{C}-\mathbf{N M R}\left(\mathbf{7 5} \mathbf{M H z}, \mathbf{C D C l}_{3}\right): \delta 11.66\left(\mathrm{CH}_{2} \underline{\mathrm{CH}} 3\right), 21.17\left(\underline{\mathrm{CH}}_{2} \mathrm{CH}_{3}\right)$, 52.82 (d, $J=6.9 \mathrm{~Hz}, \mathrm{OCH}_{3}$ ), 52.98 (d, $J=10.4 \mathrm{~Hz}, \mathrm{NCH}_{2} \mathrm{CH}_{2}$ ), 53.80 (d, $J=6.9 \mathrm{~Hz}, \mathrm{OCH}_{3}$ ), $55.31\left(\mathrm{PhOCH}_{3}\right), 55.39$ (d, $\left.J=5.8 \mathrm{~Hz}, \mathrm{NCH}_{2}\right), 58.44$ (d, $\left.J=163.8 \mathrm{~Hz}, \mathrm{CHP}\right), 81.50$ (Cㅡㅏ), 
82.20 ( $\underline{C C H}), 113.42$ (2 x CHarom), 124.19 (d, $J=12.7 \mathrm{~Hz}, \mathrm{PCHC} C$ ), $127.97\left(\mathrm{CH}_{\text {arom }}\right), 128.66$ ( $\mathrm{CH}_{\text {arom }}$ ), 129.89 (2 x CHarom), 130.82 (d, $\left.J=3.5 \mathrm{~Hz}, \mathrm{PCHC} C \mathrm{H}\right), 132.37$ (C,arom), 133.41

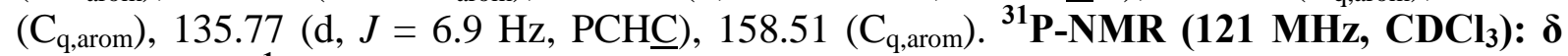
26.31. IR (cm-1) v $\mathbf{v a x}_{\text {max }} 1034$ (P-O), 1057 (P-O), 1246 (P=O), 1612 (C=C), 2099 (alkyne). MS (ESI): $\mathbf{~ m / z ~ ( \% ) : ~} 402.2\left(\mathrm{M}+\mathrm{H}^{+}, 100\right)$. MP $\left({ }^{\circ} \mathbf{C}\right): 82$. Yield: $71 \%$.

Dimethyl [(2-ethynylphenyl) -(4-phenyl-3,6-dihydro-2H-pyridin-1-yl)methyl]phosphonate 21<smiles>C#Cc1ccccc1C(POC)N1CC=C(c2ccccc2)CC1</smiles>

${ }^{1}$ H-NMR (300 MHz, $\mathbf{C D C l}_{3}$ ): $\boldsymbol{\delta}$ 2.44-2.66 (m, 2H, $\mathrm{NCH}_{2} \mathrm{CH}_{2}$ ), 2.75 (ddd, $J=4.4 \mathrm{~Hz}, J=7.3 \mathrm{~Hz}, J=11.4 \mathrm{~Hz}, 1 \mathrm{H}, \mathrm{NC}_{\mathrm{A}} \mathrm{H}_{\mathrm{B}} \mathrm{CH}_{2}$ ), 3.31 (dt, $J=4.6 \mathrm{~Hz}, J=11.4 \mathrm{~Hz}, 1 \mathrm{H}, \mathrm{NCH}_{\mathrm{A}} \underline{\mathrm{H}}_{\mathrm{B}} \mathrm{CH}_{2}$ ), 3.34 (s, $1 \mathrm{H}, \mathrm{CCH}$ ), $3.43\left(\mathrm{~d}, J=2.9 \mathrm{~Hz}, 1 \mathrm{H}, \mathrm{NC}_{\mathrm{H}_{\mathrm{A}}} \mathrm{H}_{\mathrm{B}}\right), 3.44(\mathrm{~d}, J=2.9 \mathrm{~Hz}, 1 \mathrm{H}$, $\mathrm{NCH}_{\mathrm{A}} \underline{\mathrm{H}}_{\mathrm{B}}$ ), 3.50 (d, $\left.J=10.5 \mathrm{~Hz}, 3 \mathrm{H}, \mathrm{OCH}_{3}\right), 3.88$ (d, $J=10.5 \mathrm{~Hz}$, $3 \mathrm{H}, \mathrm{OCH}_{3}$ ), 4.92 (d, $J=22.0 \mathrm{~Hz}, 1 \mathrm{H}, \mathrm{CHP}$ ), 6.02 (br s, 1H, CH), 7.18-7.41 (m, 7H, $7 \times \mathrm{CH}_{\text {arom }}$ ), 7.57 (dt, $J=7.7 \mathrm{~Hz}, J=1.1 \mathrm{~Hz}, 1 \mathrm{H}, \mathrm{PCHCC} \underline{\mathrm{H}}$ ), 7.94 (dt, $J=$ $\left.7.9 \mathrm{~Hz}, J=2.5 \mathrm{~Hz}, 1 \mathrm{H}, \mathrm{PCHCCC} \underline{\mathrm{H}}) .{ }^{13} \mathbf{C}-\mathbf{N M R}\left(75 \mathbf{M H z}, \mathbf{C D C l}_{3}\right): \boldsymbol{\delta} 28.29\left(\mathrm{NCH}_{2} \underline{\mathrm{CH}}\right)_{2}\right)$, 47.97 (d, $J=6.9 \mathrm{~Hz}, \mathrm{NCH}_{2} \mathrm{CH}_{2}$ ), 51.57 (d, $\left.J=11.5 \mathrm{~Hz}, \mathrm{NCH}_{2} \mathrm{CH}\right), 53.06$ (d, $J=8.1 \mathrm{~Hz}$, $\mathrm{OCH}_{3}$ ), 54.06 (d, $\left.J=6.9 \mathrm{~Hz}, \mathrm{OCH}_{3}\right), 63.07$ (d, $\left.J=161.5 \mathrm{~Hz}, \mathrm{CHP}\right), 82.15(\underline{\mathrm{CCH}}), 82.24$

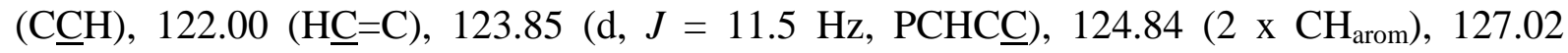
( $\left.\mathrm{CH}_{\text {arom }}\right), 128.06$ ( $\left.\mathrm{CH}_{\text {arom }}\right), 128.37$ (2 x $\left.\mathrm{CH}_{\text {arom }}\right), 128.83\left(\mathrm{CH}_{\text {arom }}\right), 130.33$ (d, $J=3.5 \mathrm{~Hz}$, PCHC태), 133.27 (PCHCCㄷH), 134.54 (C $\left.\mathrm{C}_{\mathrm{q}, \text { arom}}\right), 135.12$ (d, $\left.J=2.0 \mathrm{~Hz}, \mathrm{PCHC}\right), 140.80$

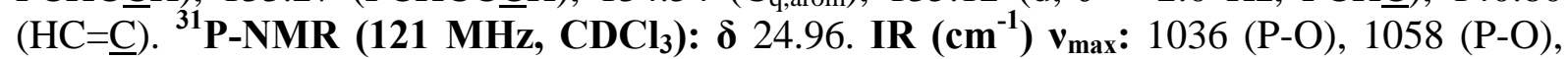
$1245(\mathrm{P}=\mathrm{O}), 1654(\mathrm{C}=\mathrm{C}), 2100$ (alkyne). MS (ESI): m/z (\%): $382.3\left(\mathrm{M}+\mathrm{H}^{+}, 100\right)$. Chromatography: Hex/EtOAc $1 / 1 \mathrm{R}_{\mathrm{f}}=0.16$. Yield: $75 \%$.

Dimethyl [(2-ethynylphenyl) -(4-(4-fluorophenyl)-3,6-dihydro-2H-pyridin-1-yl)methyl]phosphonate $2 \mathrm{~m}$<smiles>C#Cc1ccccc1C(POC)N1CC=C(c2ccc(F)cc2)CC1</smiles>

${ }^{1}$ H-NMR (300 MHz, $\left.\mathbf{C D C l}_{3}\right)$ : $\boldsymbol{\delta}$ 2.42-2.61 (m, 2H, $\left.\mathrm{NCH}_{2} \underline{\mathrm{C}}_{2}\right)$, 2.74 (ddd, $J=4.4 \mathrm{~Hz}, J=7.4 \mathrm{~Hz}, J=11.4 \mathrm{~Hz}, 1 \mathrm{H}$, $\mathrm{NC}_{\mathrm{H}_{\mathrm{A}}} \mathrm{H}_{\mathrm{B}} \mathrm{CH}_{2}$ ), 3.30 (dt, $J=3.3 \mathrm{~Hz}, J=11.4 \mathrm{~Hz}, 1 \mathrm{H}$, $\mathrm{NCH}_{\mathrm{A}} \underline{\mathrm{H}}_{\mathrm{B}} \mathrm{CH}_{2}$ ), 3.34 (s, $\left.1 \mathrm{H}, \mathrm{CCH}\right), 3.43$ (br s, $2 \mathrm{H}, \mathrm{NCH}_{2}$ ), 3.50 (d, $\left.J=10.5 \mathrm{~Hz}, 3 \mathrm{H}, \mathrm{OCH}_{3}\right), 3.88\left(\mathrm{~d}, J=10.5 \mathrm{~Hz}, 3 \mathrm{H}, \mathrm{OCH}_{3}\right)$, 4.91 (d, $J=22.0 \mathrm{~Hz}, 1 \mathrm{H}, \mathrm{CHP}$ ), 5.96 (br s, $1 \mathrm{H}, \mathrm{CH}), 6.98$ (t, $J=$ $\left.8.5 \mathrm{~Hz}, 2 \mathrm{H}, 2 \times \mathrm{CH}_{\text {arom }}\right), 7.26-7.44\left(\mathrm{~m}, 4 \mathrm{H}, 4 \times \mathrm{CH}_{\text {arom }}\right), 7.57$ (d, $\left.J=7.6 \mathrm{~Hz}, 1 \mathrm{H}, \mathrm{PCHCC} \underline{\mathrm{H}}\right)$, 7.93 (d, $J=8.0 \mathrm{~Hz}, 1 \mathrm{H}$, PCHCCC프). $\left.{ }^{13} \mathbf{C}-\mathbf{N M R}\left(75 \mathbf{M H z}, \mathbf{C D C l}_{3}\right): \delta 28.53\left(\mathrm{NCH}_{2} \underline{\mathrm{CH}}\right)_{2}\right)$, 47.91 (d, $J=8.1 \mathrm{~Hz}, \mathrm{NCH}_{2} \mathrm{CH}_{2}$ ), 51.49 (d, $\left.J=11.5 \mathrm{~Hz}, \mathrm{NCH}_{2} \mathrm{CH}\right), 53.09$ (d, $J=8.1 \mathrm{~Hz}$, $\mathrm{OCH}_{3}$ ), 54.00 (d, $\left.J=6.9 \mathrm{~Hz}, \mathrm{OCH}_{3}\right), 63.04$ (d, $\left.J=161.5 \mathrm{~Hz}, \mathrm{CHP}\right), 82.12(\underline{\mathrm{CCH}}), 82.24$ (CCLH), 115.13 (d, $\left.J=20.8 \mathrm{~Hz}, 2 \times \mathrm{CH}_{\text {arom}}\right), 121.86(\mathrm{HC}=\mathrm{C}), 123.83$ (d, $J=11.5 \mathrm{~Hz}$, PCHCC), 126.36 (d, $\left.J=20.8 \mathrm{~Hz}, 2 \times \mathrm{CH}_{\text {arom }}\right), 128.08$ ( $\left.\mathrm{CH}_{\text {arom }}\right), 128.84\left(\mathrm{CH}_{\text {arom }}\right), 130.30$ (d, $J$ $=3.5 \mathrm{~Hz}, \mathrm{PCHCCH}), 133.28(\mathrm{PCHCCCH}), 133.64(\mathrm{HC}=\underline{\mathrm{C}}), 135.11\left(\mathrm{C}_{\mathrm{q}, \text { arom }}\right), 136.91(\mathrm{~d}, J=$ $3.5 \mathrm{~Hz}, \mathrm{PCHC}), 162.06$ (d, $\left.J=245.8 \mathrm{~Hz}, \mathrm{FC}_{\mathrm{q}, \mathrm{arom}}\right) .{ }^{31} \mathbf{P}-\mathbf{N M R}\left(\mathbf{1 2 1} \mathbf{M H z}, \mathbf{C D C l}_{\mathbf{3}}\right): \boldsymbol{\delta} 24.93$.

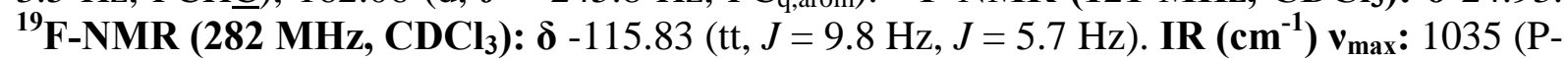
O), 1057 (P-O), 1227 ( $\mathrm{P}=\mathrm{O}), 1602$ (C=C), 2100 (alkyne). MS (ESI): m/z (\%): $400.2\left(\mathrm{M}+\mathrm{H}^{+}\right.$, 100). MP $\left({ }^{\circ} \mathbf{C}\right): 76-77$. Chromatography: Hex/EtOAc $4 / 6 \mathrm{R}_{\mathrm{f}}=0.21$. Yield: $62 \%$. 


\section{Typical procedure for the synthesis of isoindoles 4 and 14a-b.}

In a dry reaction tube, compounds $2(0.5 \mathrm{mmol})$ are dissolved into a mixture of acetonitrile $(3 \mathrm{~mL})$ and benzene $(3 \mathrm{~mL})$. This solution is heated in a microwave to $165^{\circ} \mathrm{C}$ for 60 minutes. After this the progress of the reaction is checked by ${ }^{31} \mathrm{P}$ NMR from a sample take directly from the mixture. If this reveals the presence of remaining starting material, the reaction is placed back inside the microwave and is again heated to $165^{\circ} \mathrm{C}$. After complete conversion the compound is coated on silica gel by removal of the volatiles in vacuo and purified by column chromatography.

\section{Dimethyl 2-benzyl-3-(2-phenylbut-3-enyl)-2H-isoindol-1-ylphosphonate 4a}

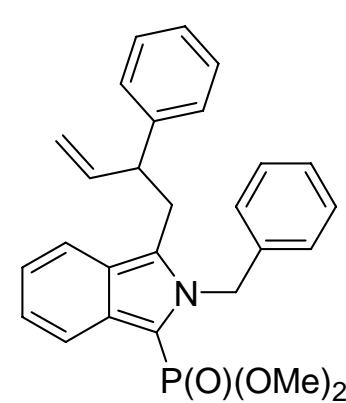

${ }^{1}$ H-NMR (300 MHz, $\mathbf{C D C l}_{3}$ ): $\boldsymbol{\delta} 3.17$ (dd, $J=7.5 \mathrm{~Hz}, J=15.1 \mathrm{~Hz}, 1 \mathrm{H}$, $\left.\mathrm{C}_{\mathrm{A}} \mathrm{H}_{\mathrm{B}} \mathrm{CH}\right), 3.43$ (dd, $\left.J=7.5 \mathrm{~Hz}, J=15.1 \mathrm{~Hz}, 1 \mathrm{H}, \mathrm{CH}_{\mathrm{A}} \underline{\mathrm{H}}_{\mathrm{B}} \mathrm{CH}\right), 3.48$ (d, $\left.J=11.6 \mathrm{~Hz}, 3 \mathrm{H}, \mathrm{OCH}_{3}\right), 3.49\left(\mathrm{~d}, J=11.6 \mathrm{~Hz}, 3 \mathrm{H}, \mathrm{OCH}_{3}\right), 3.62$ (ps q, $J$ $=7.5 \mathrm{~Hz}, \mathrm{C} \underline{\mathrm{HPh}}), 4.98\left(1 \mathrm{H}, \mathrm{d}, J=17.1 \mathrm{~Hz}, \mathrm{HC}=\mathrm{C}_{\mathrm{A}} \mathrm{H}_{\mathrm{B}}\right), 5.07(1 \mathrm{H}, \mathrm{d}, J$

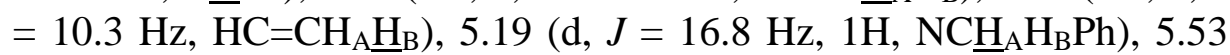
(d, $J=16.8 \mathrm{~Hz}, 1 \mathrm{H}, \mathrm{NCH}_{\mathrm{A}} \underline{\mathrm{H}}_{\mathrm{B}} \mathrm{Ph}$ ), 6.10 (ddd, $J=17.1 \mathrm{~Hz}, J=10.3 \mathrm{~Hz}, J$ $\left.=7.5 \mathrm{~Hz}, 1 \mathrm{H}, \underline{\mathrm{HC}}=\mathrm{CH}_{2}\right), 6.74-7.53\left(\mathrm{~m}, 13 \mathrm{H}, 13 \times \mathrm{CH}_{\mathrm{arom}}\right), 7.95(\mathrm{~d}, J=$ $8.5 \mathrm{~Hz}, 1 \mathrm{H}$, PCCCC $\underline{\mathrm{H}}) .{ }^{13} \mathbf{C}-\mathbf{N M R}\left(\mathbf{7 5} \mathbf{M H z}, \mathbf{C D C l}_{3}\right): \delta 32.18$ $\left(\underline{\mathrm{CH}_{2} \mathrm{CH}}\right), 49.63\left(\mathrm{NCH}_{2}\right), 50.20(\underline{\mathrm{CHPh}}), 52.33(\mathrm{~d}, J=3.5 \mathrm{~Hz}, 2 \mathrm{x}$ $\left.\mathrm{OCH}_{3}\right), 103.63$ (d, $\left.J=231.9 \mathrm{~Hz}, \mathrm{PC}\right), 115.71\left(\mathrm{HC}=\underline{C H}_{2}\right), 119.89\left(\mathrm{CH}_{\text {arom }}\right), 120.15\left(\mathrm{CH}_{\text {arom}}\right)$, $121.13\left(\mathrm{CH}_{\text {arom }}\right), 124.05$ (d, $\left.J=12.7 \mathrm{~Hz}, \mathrm{PCCC}\right), 124.84\left(\mathrm{CH}_{\text {arom }}\right), 125.74(2 \mathrm{x} \mathrm{CH}$ arom), $127.02\left(\mathrm{CH}_{\text {arom }}\right), 127.39$ ( $\left.\mathrm{CH}_{\text {arom }}\right), 127.65$ (2 x CHarom $), 128.69$ (4 x CH $\left.\mathrm{CH}_{\text {arom }}\right), 131.07$ (d, $J=9.2$ $\mathrm{Hz}, \mathrm{NC}), 133.00$ (d, $J=17.3 \mathrm{~Hz}, \mathrm{PC} \underline{\mathrm{C}}), 137.79\left(\mathrm{C}_{\mathrm{q}}, \mathrm{Ph}\right), 140.06\left(\mathrm{HC}=\mathrm{CH}_{2}\right), 142.80\left(\mathrm{C}_{\mathrm{q}}, \mathrm{Ph}\right)$. ${ }^{31}$ P-NMR (121 MHz, CDCl $\left.{ }_{3}\right): \delta$ 14.66. IR (cm $\left.{ }^{-1}\right) v_{\max }$ : $1022(\mathrm{P}-\mathrm{O}), 1049(\mathrm{P}-\mathrm{O}), 1242(\mathrm{P}=\mathrm{O})$, 1702 (C=C). MS (ESI): m/z (\%): $446.3\left(\mathrm{M}+\mathrm{H}^{+}, 100\right)$. Chromatography: Hex/EtOAc 1/1 $\mathrm{R}_{\mathrm{f}}$ $=0.34$. Yield: $82 \%$.

\section{Dimethyl 2-benzyl-3-but-3-enyl-2H-isoindol-1-ylphosphonate 4b}

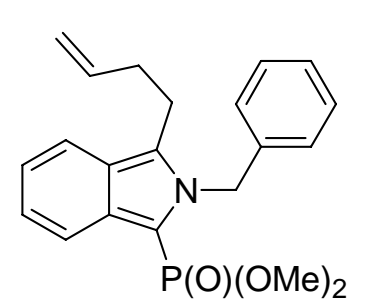

${ }^{1}$ H-NMR (300 MHz, $\mathbf{C D C l}_{3}$ ): $\boldsymbol{\delta} 2.25$ (ps q, $J=7.5 \mathrm{~Hz}, 2 \mathrm{H}, \mathrm{C}_{2} \mathrm{CH}$ ), 3.02 (t, $J=7.3 \mathrm{~Hz}, 2 \mathrm{H}, \mathrm{C}_{2} \mathrm{CH}_{2}$ ), 3.55 (d, $J=11.5 \mathrm{~Hz}, 6 \mathrm{H}, 2 \times \mathrm{OCH}_{3}$ ), $4.97\left(\mathrm{~d}, J=10.7 \mathrm{~Hz}, 1 \mathrm{H}, \mathrm{HC}=\mathrm{C}_{\mathrm{A}} \mathrm{H}_{\mathrm{B}}\right), 4.98(\mathrm{~d}, J=16.3 \mathrm{~Hz}, 1 \mathrm{H}$, $\mathrm{HC}=\mathrm{CH}_{\mathrm{A}} \underline{\mathrm{H}}_{\mathrm{B}}$ ), 5.77 (ddt, $J=7.3 \mathrm{~Hz}, J=10.7 \mathrm{~Hz}, J=16.3 \mathrm{~Hz}, 1 \mathrm{H}$, $\left.\underline{\mathrm{HC}}=\mathrm{CH}_{2}\right), 5.84\left(\mathrm{~s}, 2 \mathrm{H}, \mathrm{NCH}_{2}\right), 6.87\left(\mathrm{~d}, J=6.6 \mathrm{~Hz}, 2 \times \mathrm{CH}_{\text {arom }}\right), 7.05(\mathrm{t}$, $\left.J=7.6 \mathrm{~Hz}, \mathrm{CH}_{\text {arom }}\right), 7.17-7.29\left(\mathrm{~m}, 4 \mathrm{H}, 4 \times \mathrm{CH}_{\text {arom }}\right), 7.62(\mathrm{~d}, J=8.5 \mathrm{~Hz}$, PCCC $\underline{H}), 7.93(\mathrm{~d}, J=8.8 \mathrm{~Hz}, 1 \mathrm{H}$, PCCCC $\underline{\mathrm{H}}) .{ }^{13} \mathrm{C}-\mathrm{NMR}(\mathbf{7 5} \mathrm{MHz}$, $\left.\mathbf{C D C l}_{3}\right): \boldsymbol{\delta} 24.93\left(\mathrm{NCCH}_{2}\right), 33.97\left(\underline{\mathrm{CH}}_{2} \mathrm{CH}\right), 50.12\left(\mathrm{NCH}_{2}\right), 52.48\left(\mathrm{~d}, J=5.8 \mathrm{~Hz}, 2 \times \mathrm{OCH}_{3}\right)$, 103.57 (d, $J=234.2 \mathrm{~Hz}, \mathrm{PC}), 116.05\left(\mathrm{HC}=\mathrm{CH}_{2}\right), 119.86\left(\mathrm{CH}_{\text {arom }}\right), 120.03\left(\mathrm{CH}_{\text {arom }}\right), 121.04$ ( $\mathrm{CH}_{\text {arom }}$ ), 123.43 (d, $J=12.7 \mathrm{~Hz}$, PCCC), $124.94\left(\mathrm{CH}_{\text {arom }}\right), 125.93$ (2 x $\left.\mathrm{CH}_{\text {arom }}\right), 127.48$ ( $\mathrm{CH}_{\text {arom }}$ ), 128.72 (2 x CH arom), 132.80 (d, $\left.J=9.2 \mathrm{~Hz}, \mathrm{NC}\right), 132.82$ (d, $J=17.3 \mathrm{~Hz}, \mathrm{PC} \underline{\text { ) }}$, $137.01\left(\mathrm{HC}=\mathrm{CH}_{2}\right), 137.80\left(\mathrm{C}_{\mathrm{q}}, \mathrm{Ph}\right) .{ }^{31} \mathbf{P}-\mathbf{N M R}\left(121 \mathrm{MHz}, \mathbf{C D C l}_{3}\right): \delta$ 14.80. IR $\left(\mathbf{c m}^{-1}\right) \mathbf{v}_{\max }$ : 1023 (P-O), 1049 (P-O), 1241 (P=O), 1698 (C=C). MS (ESI): m/z (\%): $370.2\left(\mathrm{M}+\mathrm{H}^{+}, 100\right)$. Chromatography: Hex/EtOAc 55/45 $\mathrm{R}_{\mathrm{f}}=0.25$. Yield: $76 \%$. 
Dimethyl 3-[2-(4-methoxyphenyl)but-3-enyl]-2-propyl-2H-isoindol-1-ylphosphonate 4c

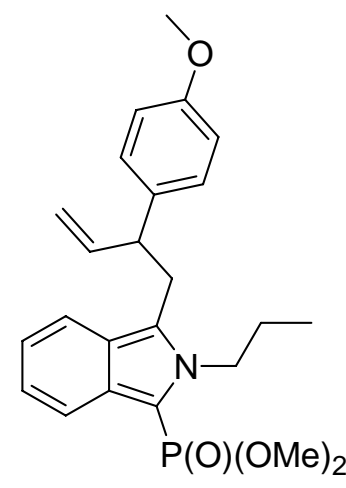

${ }^{1}$ H-NMR (300 MHz, CDCl 3 ): $\boldsymbol{\delta} 0.92$ (t, $\left.J=7.4 \mathrm{~Hz}, 3 \mathrm{H}, \mathrm{CH}_{3}\right)$, 1.66$1.80\left(\mathrm{~m}, 2 \mathrm{H}, \mathrm{C}_{2} \mathrm{CH}_{3}\right), 3.24$ (dd, $J=8.0 \mathrm{~Hz}, J=14.6 \mathrm{~Hz}, 1 \mathrm{H}$, $\mathrm{C}_{\mathrm{H}_{\mathrm{A}}} \mathrm{H}_{\mathrm{B}} \mathrm{CH}$ ), 3.47 (dd, $J=7.2 \mathrm{~Hz}, J=14.6 \mathrm{~Hz}, 1 \mathrm{H}, \mathrm{CH}_{\mathrm{A}} \underline{\mathrm{H}}_{\mathrm{B}} \mathrm{CH}$ ), 3.67 (d, $\left.J=11.8 \mathrm{~Hz}, 3 \mathrm{H}, \mathrm{OCH}_{3}\right), 3.68\left(\mathrm{~d}, J=11.6 \mathrm{~Hz}, 3 \mathrm{H}, \mathrm{OCH}_{3}\right), 3.67-3.74(\mathrm{~m}$, $1 \mathrm{H}, \mathrm{C} \underline{\mathrm{HPh}}$ ), 3.76 (s, 3H, PhOC $\underline{\mathrm{H}}_{3}$ ), 4.00 (ddd, $J=6.3 \mathrm{~Hz}, J=9.5 \mathrm{~Hz}, J$ $=14.0 \mathrm{~Hz}, 1 \mathrm{H}, \mathrm{NC} \underline{H}_{\mathrm{A}} \mathrm{H}_{\mathrm{B}}$ ), 4.22 (ddd, $J=6.3 \mathrm{~Hz}, J=9.5 \mathrm{~Hz}, J=14.0$ $\mathrm{Hz}, 1 \mathrm{H}, \mathrm{NCH}_{\mathrm{A}} \underline{\mathrm{H}}_{\mathrm{B}}$ ), 5.01 (dt, $J=1.3 \mathrm{~Hz}, J=17.1 \mathrm{~Hz}, 1 \mathrm{H}, \mathrm{HC}=\mathrm{C}_{\mathrm{H}_{\mathrm{A}}} \mathrm{H}_{\mathrm{B}}$ ), 5.08 (dt, $J=1.3 \mathrm{~Hz}, J=10.2 \mathrm{~Hz}, \mathrm{HC}=\mathrm{CH}_{\mathrm{A}} \underline{\mathrm{H}}_{\mathrm{B}}$ ), 6.11 (ddd, $J=17.1 \mathrm{~Hz}$, $\left.J=10.2 \mathrm{~Hz}, J=7.0 \mathrm{~Hz}, 1 \mathrm{H}, \underline{\mathrm{HC}}=\mathrm{CH}_{2}\right), 6.74-7.51\left(\mathrm{~m}, 7 \mathrm{H}, 7 \times \mathrm{CH}_{\text {arom }}\right.$ ), $7.85(\mathrm{~d}, J=8.5 \mathrm{~Hz}, 1 \mathrm{H}$, PCCCC $\underline{\mathrm{H}}) .{ }^{13} \mathbf{C}-\mathbf{N M R}\left(\mathbf{7 5} \mathbf{M H z}, \mathbf{C D C l}_{3}\right): \boldsymbol{\delta}$ $11.38\left(\mathrm{CH}_{2} \underline{\mathrm{CH}} 3\right), 25.58\left(\mathrm{CH}_{2} \mathrm{CH}_{3}\right), 32.22\left(\underline{\mathrm{CH}}_{2} \mathrm{CH}\right), 48.25\left(\mathrm{NCH}_{2}\right)$, 49.39 ( $\underline{\mathrm{CHPh}}$ ), 52.37 (d, $\left.J=5.8 \mathrm{~Hz}, 2 \times \mathrm{OCH}_{3}\right), 55.34\left(\mathrm{PhOCH}_{3}\right), 102.33$ (d, $J=234.2 \mathrm{~Hz}$, PC), 114.05 (2 x CHarom), $115.30\left(\mathrm{HC}=\mathrm{CH}_{2}\right), 119.77$ (2 x CH arom $), 120.69\left(\mathrm{CH}_{\text {arom }}\right), 123.77$ (d, $J=13.8 \mathrm{~Hz}$, PCCC $), 124.52\left(\mathrm{CH}_{\text {arom }}\right), 128.54$ (2 x CH $\left.\mathrm{CH}_{\text {arom }}\right), 130.41$ (d, $\left.J=9.2 \mathrm{~Hz}, \mathrm{NC}\right)$, 132.64 (d, $J=18.5 \mathrm{~Hz}, \mathrm{PC} \underline{)}), 134.84\left(\mathrm{C}_{\mathrm{q}}, \mathrm{Ph}\right), 140.51\left(\mathrm{HC}=\mathrm{CH}_{2}\right), 158.60\left(\mathrm{C}_{\mathrm{q}}, \mathrm{Ph}\right) .{ }^{31} \mathbf{P}-\mathbf{N M R}$ (121 MHz, CDCl $\left.)_{3}\right): \delta$ 15.23. IR (cm $\left.{ }^{-1}\right) \mathbf{v}_{\text {max }}$ : 1024 (P-O), 1049 (P-O), $1246(\mathrm{P}=\mathrm{O}), 1698$ $(\mathrm{C}=\mathrm{C})$. MS (ESI): $\mathbf{m} / \mathbf{z}(\%): 428.3\left(\mathrm{M}+\mathrm{H}^{+}, 100\right)$. Chromatography: Hex/EtOAc $1 / 1 \mathrm{R}_{\mathrm{f}}=$ 0.15 . Yield: $68 \%$.

Dimethyl 3-(2,2-dimethylbut-3-enyl)-2-(4-methylbenzyl)-2H-isoindol-1-ylphosphonate 4d

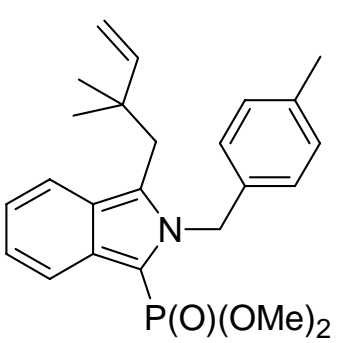

${ }^{1}$ H-NMR (300 MHz, $\left.\mathbf{C D C l}_{3}\right): \boldsymbol{\delta} 1.12$ (s, 6H, 2 x $\left.\mathrm{CH}_{3}\right), 2.27$ (s, 3H, $\left.\mathrm{CH}_{3}\right), 3.47$ (d, $\left.J=11.5 \mathrm{~Hz}, 6 \mathrm{H}, 2 \times \mathrm{OCH}_{3}\right), 4.93$ (dd, $J=17.4 \mathrm{~Hz}, J=$ $\left.1.1 \mathrm{~Hz}, 1 \mathrm{H}, \mathrm{HC}=\mathrm{C}_{\mathrm{A}} \mathrm{H}_{\mathrm{B}}\right), 4.97(\mathrm{dd}, J=10.7 \mathrm{~Hz}, J=1.1 \mathrm{~Hz}, 1 \mathrm{H}$, $\left.\mathrm{HC}=\mathrm{CH}_{\mathrm{A}} \underline{\mathrm{H}}_{\mathrm{B}}\right), 5.82\left(\mathrm{~s}, 2 \mathrm{H}, \mathrm{NCH}_{2}\right), 5.84(\mathrm{dd}, J=10.7 \mathrm{~Hz}, J=17.4 \mathrm{~Hz}$, $\left.1 \mathrm{H}, \underline{\mathrm{HC}}=\mathrm{CH}_{2}\right), 6.58\left(\mathrm{~d}, J=7.8 \mathrm{~Hz}, 2 \times \mathrm{CH}_{\text {arom }}\right), 7.02(\mathrm{~d}, J=7.8 \mathrm{~Hz}, 2 \mathrm{x}$ $\mathrm{CH}_{\text {arom }}$ ), 7.05-7.21 (m, 2H, $2 \times \mathrm{CH}_{\text {arom }}$ ), 7.62-7.65 (m, 1H, PCCC $\underline{\mathrm{H}}$ ), $7.93(\mathrm{~d}, J=8.8 \mathrm{~Hz}, 1 \mathrm{H}, \mathrm{PCCCC} \underline{\mathrm{H}}) .{ }^{13} \mathbf{C}-\mathbf{N M R}\left(75 \mathbf{M H z}, \mathbf{C D C l}_{3}\right): \boldsymbol{\delta}$ $21.09\left(\mathrm{CH}_{3}\right), 27.57\left(2 \times \mathrm{CH}_{3}\right), 37.52\left(\mathrm{NCCH}_{2}\right), 39.81\left(\mathrm{C}\left(\mathrm{CH}_{3}\right)_{2}\right), 50.21$ $\left(\mathrm{NCH}_{2}\right), 52.30$ (d, $\left.J=4.6 \mathrm{~Hz}, 2 \times \mathrm{OCH}_{3}\right), 104.03$ (d, $\left.J=233.1 \mathrm{~Hz}, \mathrm{PC}\right), 111.82\left(\mathrm{HC}=\underline{\mathrm{CH}}_{2}\right)$, $120.02\left(\mathrm{CH}_{\text {arom }}\right), 120.93$ ( $\left.\mathrm{CH}_{\text {arom }}\right), 121.19\left(\mathrm{CH}_{\text {arom }}\right), 124.64\left(\mathrm{CH}_{\text {arom }}\right), 123.94$ (d, $J=12.7 \mathrm{~Hz}$, PCCㅡ), 125.50 ( $\left.2 \times \mathrm{CH}_{\text {arom }}\right), 129.28$ (2 x CH arom), 130.86 (d, $\left.J=9.2 \mathrm{~Hz}, \mathrm{NC}\right), 132.11$ (d, $J=$ $17.3 \mathrm{~Hz}, \mathrm{PC} \underline{\mathrm{C}}), 134.84\left(\mathrm{C}_{\mathrm{q}}, \mathrm{Ph}\right), 136.95\left(\mathrm{C}_{\mathrm{q}}, \mathrm{Ph}\right), 147.04\left(\mathrm{HC}=\mathrm{CH}_{2}\right) .{ }^{31} \mathbf{P}-\mathbf{N M R}(\mathbf{1 2 1} \mathbf{~ M H z}$, $\left.\mathbf{C D C l}_{3}\right): \boldsymbol{\delta}$ 14.79. IR (cm $\left.{ }^{-1}\right) \mathbf{v}_{\max }: 1023(\mathrm{P}-\mathrm{O}), 1051(\mathrm{P}-\mathrm{O}), 1243(\mathrm{P}=\mathrm{O}), 1638(\mathrm{C}=\mathrm{C}) . \mathbf{M S}$ (ESI): m/z (\%): $412.3\left(\mathrm{M}+\mathrm{H}^{+}, 100\right)$. Chromatography: Hex/EtOAc $1 / 1 \mathrm{R}_{\mathrm{f}}=0.22$. Yield: $47 \%$.

\section{Dimethyl 3-[2-isopropylbut-3-enyl]-2-(2-fenylethyl)-2H-isoindol-1-ylphosphonate 4e}

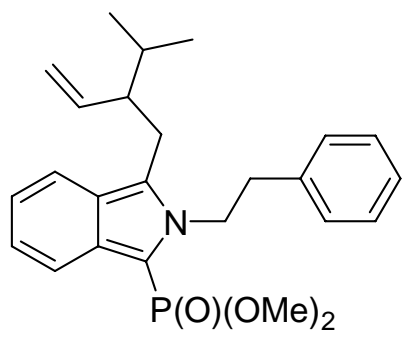

${ }^{1}$ H-NMR (300 MHz, $\left.\mathbf{C D C l}_{3}\right): \boldsymbol{\delta} 0.95$ (d, $J=6.4 \mathrm{~Hz}, 3 \mathrm{H}, \mathrm{CH}_{3}$ ), 0.96 (d, $J=6.4 \mathrm{~Hz}, 3 \mathrm{H}, \mathrm{CH}_{3}$ ), 1.70 (octet, $J=6.4 \mathrm{~Hz}, 1 \mathrm{H}$, $\left.\mathrm{C} \underline{\mathrm{H}}\left(\mathrm{CH}_{3}\right)_{2}\right), 2.24$ (tt, $\left.J=6.4 \mathrm{~Hz}, J=9.8 \mathrm{~Hz}, 1 \mathrm{H}, \mathrm{CH}_{2} \mathrm{CH}\right), 2.76$ (dd, $\left.J=9.8 \mathrm{~Hz}, J=14.9 \mathrm{~Hz}, 1 \mathrm{H}, \mathrm{CH}_{\mathrm{A}} \mathrm{H}_{\mathrm{B}} \mathrm{CH}\right), 2.98(\mathrm{dd}, J=6.4 \mathrm{~Hz}, J=$ $\left.14.9 \mathrm{~Hz}, 1 \mathrm{H}, \mathrm{CH}_{\mathrm{A}} \underline{\mathrm{H}}_{\mathrm{B}} \mathrm{CH}\right), 3.05-3.20\left(\mathrm{~m}, 2 \mathrm{H}, \mathrm{CH}_{2} \mathrm{Ph}\right), 3.74$ (d, $J=$ $11.6 \mathrm{~Hz}, 3 \mathrm{H}, \mathrm{OCH}_{3}$ ), 3.75 (d, $J=11.5 \mathrm{~Hz}, 3 \mathrm{H}, \mathrm{OCH}_{3}$ ), 4.61 (dd, $J$ $\left.=1.7 \mathrm{~Hz}, J=17.1 \mathrm{~Hz}, 1 \mathrm{H}, \mathrm{HC}=\mathrm{C}_{\mathrm{A}} \mathrm{H}_{\mathrm{B}}\right), 4.61-4.79\left(\mathrm{~m}, 2 \mathrm{H}, \mathrm{NCH}_{2}\right)$, 4.82 (dt, $J=1.7 \mathrm{~Hz}, J=9.8 \mathrm{~Hz}, \mathrm{HC}=\mathrm{CH}_{\mathrm{A}} \underline{\mathrm{H}}_{\mathrm{B}}$ ), 5.63 (dt, $J=17.1$ 
$\left.\mathrm{Hz}, J=9.8 \mathrm{~Hz}, 1 \mathrm{H}, \underline{\mathrm{HC}}=\mathrm{CH}_{2}\right), 6.98-7.33\left(\mathrm{~m}, 7 \mathrm{H}, 7 \times \mathrm{CH}_{\text {arom }}\right), 7.52(\mathrm{~d}, J=8.5 \mathrm{~Hz}, 1 \mathrm{H}$, PCCCH $\underline{H}), 7.86(\mathrm{~d}, J=8.8 \mathrm{~Hz}, 1 \mathrm{H}, \mathrm{PCCCCH}) .{ }^{13} \mathbf{C}-\mathbf{N M R}\left(\mathbf{7 5} \mathbf{M H z}, \mathbf{C D C l}_{3}\right): \boldsymbol{\delta} 18.85\left(\mathrm{CH}_{3}\right)$, $20.90\left(\mathrm{CH}_{3}\right), 27.90\left(\underline{\mathrm{CH}_{2} \mathrm{CH}}\right), 31.46\left(\underline{\mathrm{CH}}\left(\mathrm{CH}_{3}\right)_{2}\right), 38.88\left(\underline{\mathrm{C}} \mathrm{H}_{2} \mathrm{Ph}\right), 48.90\left(\mathrm{NCH}_{2}\right), 51.51$ $\left(\underline{\mathrm{CHCH}}=\mathrm{CH}_{2}\right), 52.53\left(\mathrm{~d}, J=4.6 \mathrm{~Hz}, 2 \times \mathrm{OCH}_{3}\right), 101.81(\mathrm{~d}, J=234.2 \mathrm{~Hz}, \mathrm{PC}), 116.76$ $\left(\mathrm{HC}=\underline{\mathrm{CH}}_{2}\right), 119.60\left(\mathrm{CH}_{\text {arom }}\right), 120.14\left(\mathrm{CH}_{\text {arom }}\right), 120.54\left(\mathrm{CH}_{\text {arom }}\right), 123.73(\mathrm{~d}, J=12.7 \mathrm{~Hz}$, PCCㅡ), 124.73 ( $\left.\mathrm{CH}_{\text {arom }}\right), 126.90$ ( $\left.\mathrm{CH}_{\text {arom }}\right), 128.81$ (2 x CH arom $), 129.01$ (2 x $\left.\mathrm{CH}_{\text {arom }}\right), 132.13$ (d, $J=10.4 \mathrm{~Hz}, \mathrm{NC}), 132.73$ (d, $J=17.3 \mathrm{~Hz}, \mathrm{PC} \underline{C}), 138.23\left(\mathrm{C}_{\mathrm{q}}, \mathrm{Ph}\right), 138.58\left(\mathrm{HC}=\mathrm{CH}_{2}\right) .{ }^{31} \mathbf{P}-$ NMR (121 MHz, CDCl $\left.\mathbf{l}_{3}\right): \delta$ 15.44. IR (cm $\left.{ }^{-1}\right) \mathbf{v}_{\max }$ : 1025 (P-O), $1049(\mathrm{P}-\mathrm{O}), 1247(\mathrm{P}=\mathrm{O})$, 1660 (C=C). MS (ESI): m/z (\%): $426.2\left(\mathrm{M}+\mathrm{H}^{+}, 100\right)$. Chromatography: Hex/EtOAc 1/1 $\mathrm{R}_{\mathrm{f}}$ $=0.32$. Yield: $71 \%$.

\section{Dimethyl 3-but-2-enyl-2-(3-fluorobenzyl)-2H-isoindol-1-ylphosphonate 4f}

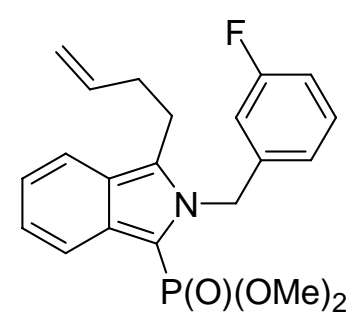

${ }^{1}$ H-NMR (300 MHz, $\left.\mathbf{C D C l}_{3}\right): \boldsymbol{\delta} 2.26$ (ps q, $J=7.4 \mathrm{~Hz}, 2 \mathrm{H}, \mathrm{C}_{2} \mathrm{CH}$ ), 3.01 (t, $\left.J=7.4 \mathrm{~Hz}, 2 \mathrm{H}, \mathrm{NCC} \underline{\mathrm{H}}_{2}\right), 3.59$ (d, $J=11.6 \mathrm{~Hz}, 6 \mathrm{H}, 2 \times \mathrm{OCH}_{3}$ ), 4.95-5.01 (m, $\left.2 \mathrm{H}, \mathrm{HC}=\mathrm{C}_{2}\right), 5.77$ (ddt, $J=7.4 \mathrm{~Hz}, J=9.9 \mathrm{~Hz}, J=17.3$ $\left.\mathrm{Hz}, 1 \mathrm{H}, \underline{\mathrm{HC}}=\mathrm{CH}_{2}\right), 5.85\left(\mathrm{~s}, 2 \mathrm{H}, \mathrm{NC}_{2} \mathrm{Ph}\right), 6.57(\mathrm{~d}, J=9.6 \mathrm{~Hz}, 1 \mathrm{H}$, $\mathrm{CH}_{\text {arom }}$ ), 6.69 (d, $\left.J=7.7 \mathrm{~Hz}, 1 \mathrm{H}, \mathrm{CH}_{\text {arom }}\right), 6.92$ (dt, $J=8.4 \mathrm{~Hz}, J=0.8$ $\mathrm{Hz}, 1 \mathrm{H}, \mathrm{CH}_{\text {arom }}$ ), 7.04-7.26 (m, 3H, 3 x $\mathrm{CH}_{\text {arom }}$ ), 7.62 (d, $J=8.5 \mathrm{~Hz}, 1 \mathrm{H}$, PCCC $\underline{H}), 7.90$ (d, $J=8.8 \mathrm{~Hz}, 1 \mathrm{H}$, PCCCC $\underline{\mathrm{H}}) .{ }^{13} \mathbf{C}-\mathbf{N M R}(\mathbf{7 5} \mathbf{M H z}$, $\left.\mathbf{C D C l}_{3}\right): \delta 24.88\left(\mathrm{NCCH}_{2}\right), 33.97\left(\underline{\mathrm{CH}}_{2} \mathrm{CH}\right), 49.60\left(\mathrm{NCH}_{2}\right), 52.52\left(\mathrm{~d}, J=5.8 \mathrm{~Hz}, 2 \times \mathrm{OCH}_{3}\right)$, 103.69 (d, $J=234.2 \mathrm{~Hz}, \mathrm{PC}$ ), 113.04 (d, $J=23.1 \mathrm{~Hz}, \mathrm{CH}_{\text {arom }}$ ), 114.45 (d, $J=21.9 \mathrm{~Hz}$, $\left.\mathrm{CH}_{\text {arom }}\right), 116.18\left(\mathrm{HC}=\underline{\mathrm{CH}}_{2}\right), 119.89\left(\mathrm{CH}_{\text {arom }}\right), 119.96\left(\mathrm{CH}_{\text {arom }}\right), 121.21\left(\mathrm{CH}_{\text {arom }}\right), 121.58(\mathrm{~d}, J$ $3.5 \mathrm{~Hz}, \mathrm{CH}_{\text {arom }}$ ), 123.48 (d, $\left.J=13.9 \mathrm{~Hz}, \mathrm{PCC} \underline{\mathrm{C}}\right), 125.13\left(\mathrm{CH}_{\text {arom }}\right), 130.30$ (d, $J=8.1 \mathrm{~Hz}$, $\mathrm{CH}_{\text {arom}}$ ), 132.64 (d, $\left.J=17.3 \mathrm{~Hz}, \mathrm{NC}\right), 132.70$ (d, $J=17.3 \mathrm{~Hz}, \mathrm{PC} \underline{\mathrm{C}}$ ), $136.86\left(\mathrm{HC}=\mathrm{CH}_{2}\right)$, 140.49 (d, $\left.J=6.9 \mathrm{~Hz}, \mathrm{C}_{\mathrm{q}, \text { arom }}\right), 163.23$ (d, $\left.J=245.8 \mathrm{~Hz}, \mathrm{FC}_{\mathrm{q}, \text { arom }}\right) .{ }^{31}$ P-NMR (121 MHz, $\left.\mathbf{C D C l}_{3}\right): \delta 1_{14.56}{ }^{19} \mathbf{F}-\mathbf{N M R}\left(\mathbf{2 8 2} \mathbf{~ M H z}, \mathbf{C D C l}_{3}\right): \boldsymbol{\delta}-112.48(\mathrm{dt}, J=5.3 \mathrm{~Hz}, J=7.2 \mathrm{~Hz}) . \mathbf{I R}$ $\left(\mathbf{c m}^{-1}\right) \mathbf{v}_{\max }: 1025$ (P-O), 1050 (P-O), 1243 (P=O), 1617 (C=C). MS (ESI): m/z (\%): 388.3 $\left(\mathrm{M}+\mathrm{H}^{+}, 100\right)$. Chromatography: Hex/EtOAc $4 / 6 \mathrm{R}_{\mathrm{f}}=0.36$. Yield: $63 \%$.

Dimethyl [2-[2-(4-chlorophenyl)-ethyl]-3-(2-methylene-cyclohexylmethyl)--2H-isoindol1-yl]-phosphonate 4g

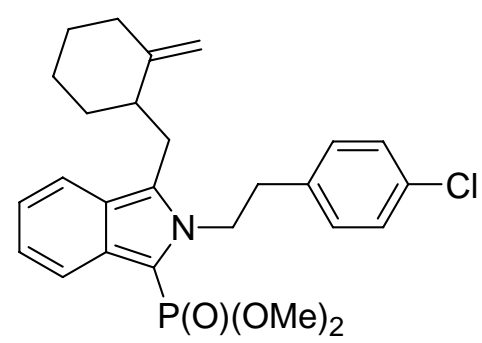

${ }^{1}$ H-NMR (300 MHz, $\left.\mathbf{C D C l}_{3}\right): \boldsymbol{\delta}$ 1.12-1.70 (m, 6H, $\left.3 \times \mathrm{CH}_{2}\right)$, 1.99-2.10 (m, 1H, $\left.\mathrm{C}_{\mathrm{A}} \mathrm{H}_{\mathrm{B}}\right), 2.30-2.38\left(\mathrm{~m}, 2 \mathrm{H}, \mathrm{CH}_{2} \mathrm{C} \underline{\mathrm{H}}+\right.$ $\mathrm{CH}_{\mathrm{A}} \underline{\mathrm{H}}_{\mathrm{B}}$ ), 2.88 (dd, $J=10.3 \mathrm{~Hz}, J=14.7 \mathrm{~Hz}, 1 \mathrm{H}, \mathrm{NCC}_{\underline{\mathrm{H}}_{\mathrm{A}}} \overline{\mathrm{H}}_{\mathrm{B}}$ ), 3.03 (dd, $J=4.3 \mathrm{~Hz}, J=14.7 \mathrm{~Hz}, 1 \mathrm{H}, \mathrm{NCCH}_{\mathrm{A}} \underline{\mathrm{H}}_{\mathrm{B}}$ ), 3.09-3.17 (m, $\left.2 \mathrm{H}, \mathrm{CH}_{2} \mathrm{Ph}\right), 3.77$ (d, $\left.J=11.5 \mathrm{~Hz}, 3 \mathrm{H}, \mathrm{OCH}_{3}\right), 3.78$ (d, $J=$ $\left.11.5 \mathrm{~Hz}, 3 \mathrm{H}, \mathrm{OCH}_{3}\right), 4.62\left(1 \mathrm{H}, \mathrm{s}, \mathrm{C}=\mathrm{CH}_{\mathrm{A}} \mathrm{H}_{\mathrm{B}}\right), 4.64(\mathrm{dt}, J=8.0$ $\left.\mathrm{Hz}, J=13.8 \mathrm{~Hz}, 1 \mathrm{H}, \mathrm{NC}_{\mathrm{A}} \mathrm{H}_{\mathrm{B}}\right), 4.74(\mathrm{dt}, J=7.9 \mathrm{~Hz}, J=13.8$ $\left.\mathrm{Hz}, 1 \mathrm{H}, \mathrm{NCH}_{\mathrm{A}} \underline{\mathrm{H}}_{\mathrm{B}}\right), 4.76\left(1 \mathrm{H}, \mathrm{s}, \mathrm{C}=\mathrm{CH}_{\mathrm{A}} \underline{\mathrm{H}}_{\mathrm{B}}\right), 6.99-7.28(\mathrm{~m}, 6 \mathrm{H}, 6$ x CH $\left.\mathrm{CH}_{\text {arom }}\right), 7.53$ (d, $\left.J=8.5 \mathrm{~Hz}, 1 \mathrm{H}, \mathrm{PCCCH}\right), 7.82(\mathrm{~d}, J=8.5 \mathrm{~Hz}, 1 \mathrm{H}$, PCCCC $\underline{\mathrm{H}}) \cdot{ }^{13} \mathbf{C}-\mathbf{N M R}$ (75 MHz, CDCl $\left.)_{3}\right): \delta 24.97\left(\mathrm{CH}_{2}\right), 27.90\left(\mathrm{NCCH}_{2}\right), 28.47\left(\mathrm{CH}_{2}\right), 33.39\left(\mathrm{CH}_{2}\right), 35.75\left(\mathrm{CH}_{2}\right)$, $38.23\left(\mathrm{NCH}_{2} \mathrm{CH}_{2}\right), 43.49(\mathrm{CH}), 48.53\left(\mathrm{NCH}_{2}\right), 52.68\left(\mathrm{~d}, J=3.5 \mathrm{~Hz}, 2 \times \mathrm{OCH}_{3}\right), 102.27$ (d, $J$ $=235.4 \mathrm{~Hz}, \mathrm{PC}), 105.92\left(\mathrm{C}=\underline{\mathrm{CH}}_{2}\right), 119.45\left(\mathrm{CH}_{\text {arom }}\right), 120.22\left(\mathrm{CH}_{\text {arom }}\right), 120.78\left(\mathrm{CH}_{\text {arom }}\right), 123.93$ (d, $J=12.7 \mathrm{~Hz}$, PCCC), $124.93\left(\mathrm{CH}_{\text {arom }}\right), 128.89$ (2 x CHarom), 130.44 (2 x $\left.\mathrm{CH}_{\text {arom }}\right), 132.02$ (d, $J=9.2 \mathrm{~Hz}, \mathrm{NC}), 132.45$ (d, $J=17.3 \mathrm{~Hz}, \mathrm{PC}$ ), 132.78 (ClC $\left.\mathrm{Cl}_{\mathrm{q}, \mathrm{rrom}}\right), 136.57$ (C,arom), 152.22 $\left(\underline{\mathrm{C}}=\mathrm{CH}_{2}\right) .{ }^{31} \mathrm{P}-\mathrm{NMR}\left(121 \mathrm{MHz}, \mathbf{C D C l}_{3}\right): \delta$ 15.25. IR $\left(\mathbf{c m}^{-1}\right) \mathbf{v}_{\max }: 1024(\mathrm{P}-\mathrm{O}), 1049$ (P-O),

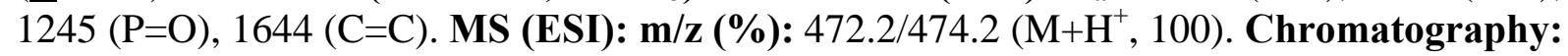
Hex/EtOAc $1 / 1 \mathrm{R}_{\mathrm{f}}=0.26$. Yield: $40 \%$. 
Dimethyl 2-butyl-3-(3-methyl-2-phenylbut-3-enyl)-2H-isoindol-1-ylphosphonate $4 \mathrm{~h}$

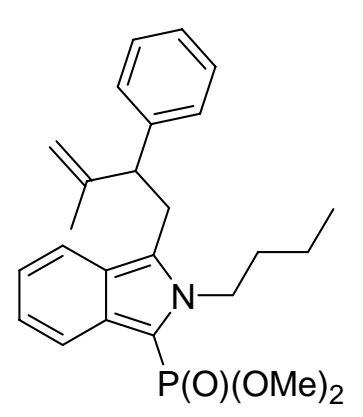

${ }^{1}$ H-NMR (300 MHz, $\mathbf{C D C l}_{3}$ ): $\boldsymbol{\delta} 0.89$ (t, $\left.J=7.6 \mathrm{~Hz}, 3 \mathrm{H}, \mathrm{CH}_{3}\right), 1.29$ (sextet, $\left.J=7.6 \mathrm{~Hz}, 2 \mathrm{H}, \mathrm{C}_{2} \mathrm{CH}_{3}\right), 1.59$ (p, $J=7.6 \mathrm{~Hz}, 2 \mathrm{H}, \mathrm{NCH}_{2} \underline{\mathrm{C}}_{2}$ ), 1.66 (s, 3H, $\mathrm{CH}_{3}$ ), 3.22 (dd, $J=9.5 \mathrm{~Hz}, J=13.3 \mathrm{~Hz}, 1 \mathrm{H}, \mathrm{C}_{\mathrm{A}} \mathrm{H}_{\mathrm{B}} \mathrm{CH}$ ), 3.54-3.77 (m, 3H, $\left.\mathrm{CH}_{\mathrm{A}} \underline{\mathrm{H}}_{\mathrm{B}} \mathrm{C} \underline{\mathrm{H}}+\mathrm{NC}_{\mathrm{A}} \mathrm{H}_{\mathrm{B}}\right), 3.64(\mathrm{~d}, J=11.5 \mathrm{~Hz}, 3 \mathrm{H}$, $\mathrm{OCH}_{3}$ ), 3.65 (d, $J=11.6 \mathrm{~Hz}, 3 \mathrm{H}, \mathrm{OCH}_{3}$ ), 4.10 (dt, $J=7.6 \mathrm{~Hz}, J=14.0$ $\left.\mathrm{Hz}, \quad 1 \mathrm{H}, \quad \mathrm{NCH}_{\mathrm{A}} \underline{\mathrm{H}}_{\mathrm{B}}\right), 5.08 \quad\left(\mathrm{~s}, 1 \mathrm{H}, \quad \mathrm{HC}=\mathrm{C}_{\mathrm{H}} \mathrm{H}_{\mathrm{B}}\right), 5.11 \quad(\mathrm{~s}, 1 \mathrm{H}$, $\left.\mathrm{HC}=\mathrm{CH}_{\mathrm{A}} \underline{\mathrm{H}}_{\mathrm{B}}\right), 6.85-7.20\left(\mathrm{~m}, 7 \mathrm{H}, 7 \times \mathrm{CH}_{\text {arom }}\right), 7.42(\mathrm{~d}, J=8.3 \mathrm{~Hz}, 1 \mathrm{H}$, PCCC $\underline{H}), 7.86(\mathrm{~d}, J=8.5 \mathrm{~Hz}, 1 \mathrm{H}$, PCCCC $\underline{\mathrm{H}}) .{ }^{13} \mathrm{C}-\mathrm{NMR}(\mathbf{7 5} \mathbf{~ M H z}$, $\left.\mathbf{C D C l}_{3}\right)$ : $\boldsymbol{\delta} 13.77\left(\mathrm{CH}_{2} \underline{\mathrm{CH}} \mathrm{H}_{3}\right), 20.17\left(\underline{\mathrm{CH}}_{2} \mathrm{CH}_{3}\right), 22.79\left(\mathrm{CCH}_{3}\right), 30.83$ ( $\left.\underline{\mathrm{CH}}_{2} \mathrm{CH}\right), 34.12\left(\mathrm{NCH}_{2} \underline{\mathrm{CH}}_{2}\right), 46.13\left(\mathrm{NCH}_{2}\right), 52.36$ (d, $\left.J=3.5 \mathrm{~Hz}, 2 \times \mathrm{OCH}_{3}\right), 52.87(\underline{\mathrm{CHPh}})$, 101.90 (d, $J=234.2 \mathrm{~Hz}, \mathrm{P} \underline{\mathrm{C}}), 111.05\left(\mathrm{C}=\mathrm{CH}_{2}\right), 119.54\left(\mathrm{CH}_{\text {arom }}\right), 119.82\left(\mathrm{CH}_{\text {arom }}\right), 120.58$ $\left(\mathrm{CH}_{\text {arom }}\right), 123.63$ (d, $J=12.7 \mathrm{~Hz}$, PCCC $), 124.46\left(\mathrm{CH}_{\text {arom }}\right), 127.01\left(\mathrm{CH}_{\text {arom }}\right), 127.82$ (2 x $\mathrm{CH}_{\text {arom }}$ ), 128.44 ( $\left.2 \mathrm{x} \mathrm{CH}_{\text {arom }}\right), 130.75$ (d, $J=9.2 \mathrm{~Hz}, \mathrm{NC}$ ), 132.77 (d, $J=17.3 \mathrm{~Hz}, \mathrm{PC} \underline{\text { ) }}$, $141.86\left(\mathrm{C}_{\mathrm{q}}, \mathrm{Ph}\right), 147.09\left(\underline{\mathrm{C}}=\mathrm{CH}_{2}\right) .{ }^{31} \mathbf{P}-\mathbf{N M R}\left(\mathbf{1 2 1} \mathbf{M H z}, \mathbf{C D C l}_{3}\right): \boldsymbol{\delta} 15.24 . \mathbf{I R}\left(\mathbf{c m}^{-1}\right) \mathbf{v}_{\max }$ : 1022 (P-O), 1048 (P-O), 1251 (P=O), 1701 (C=C). MS (ESI): m/z (\%): $426.5\left(\mathrm{M}+\mathrm{H}^{+}, 100\right)$. Chromatography: Hex/EtOAc $1 / 1 \mathrm{R}_{\mathrm{f}}=0.20$. Yield: $40 \%$.

\section{Dimethyl 2-allyl-3-but-3-enyl-2H-isoindol-1-ylphosphonate 4i}

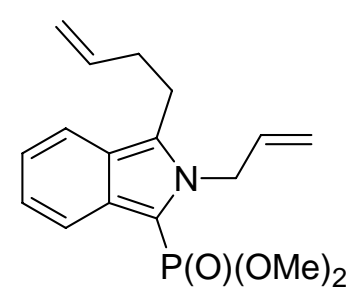

${ }^{1}$ H-NMR (300 MHz, $\left.\mathbf{C D C l}_{3}\right)$ : $\boldsymbol{\delta} 2.43$ (ps q, $J=7.4 \mathrm{~Hz}, 2 \mathrm{H}$, $\mathrm{CH}_{2} \underline{\mathrm{C}}_{2} \mathrm{CH}$ ), 3.07 (t, $\left.J=7.4 \mathrm{~Hz}, 2 \mathrm{H}, \underline{\mathrm{CH}}_{2} \mathrm{CH}_{2}\right), 3.71$ (d, $J=11.6 \mathrm{~Hz}$, $6 \mathrm{H}, 2 \times \mathrm{OCH}_{3}$ ), 4.74 (br d, $J=17.1 \mathrm{~Hz}, J=1.5 \mathrm{~Hz}, 1 \mathrm{H}$, $\mathrm{NCH}_{2} \mathrm{HC}=\mathrm{C}_{\mathrm{H}_{\mathrm{A}}} \mathrm{H}_{\mathrm{B}}$ ), 5.03 (br d, $J=10.3 \mathrm{~Hz}, 1 \mathrm{H}, \mathrm{HC}=\mathrm{C}_{\mathrm{H}_{\mathrm{A}}} \mathrm{H}_{\mathrm{B}}$ ), 5.09 (br d, $\left.J=17.1 \mathrm{~Hz}, 1 \mathrm{H}, \mathrm{HC}=\mathrm{CH}_{\mathrm{A}} \underline{\mathrm{H}}_{\mathrm{B}}\right), 5.16(\mathrm{dq}, J=10.5 \mathrm{~Hz}, J=1.5 \mathrm{~Hz}, 1 \mathrm{H}$, $\left.\mathrm{NCH}_{2} \mathrm{HC}=\mathrm{CH}_{\mathrm{A}} \underline{\mathrm{H}}_{\mathrm{B}}\right), 5.23\left(\mathrm{dt}, J=1.5 \mathrm{~Hz}, J=4.8 \mathrm{~Hz}, 2 \mathrm{H}, \mathrm{NCH}_{2}\right), 5.86$ (ddt, $J=7.4 \mathrm{~Hz}, J=10.3 \mathrm{~Hz}, J=17.1 \mathrm{~Hz}, 1 \mathrm{H}, \underline{\mathrm{HC}}=\mathrm{CH}_{2}$ ), 6.03 (ddt, $J=$ $\left.4.8 \mathrm{~Hz}, J=10.5 \mathrm{~Hz}, J=17.1 \mathrm{~Hz}, 1 \mathrm{H}, \mathrm{NCH}_{2} \underline{\mathrm{HC}}=\mathrm{CH}_{2}\right), 7.00-7.06\left(\mathrm{~m}, 1 \mathrm{H}, \mathrm{CH}_{\text {arom }}\right), 7.15-7.20$ (m, 1H, $\mathrm{CH}_{\text {arom }}$ ), 7.15-7.20 (m, 1H, $\left.\mathrm{CH}_{\text {arom }}\right), 7.58-7.62$ (m, $\left.1 \mathrm{H}, \mathrm{CH}_{\text {arom }}\right), 7.87$ (d, J = $8.5 \mathrm{~Hz}$, $1 \mathrm{H}, \mathrm{PCCCC} \underline{\mathrm{H}}) .{ }^{13} \mathbf{C}-\mathrm{NMR}\left(\mathbf{7 5} \mathbf{M H z}, \mathbf{C D C l}_{\mathbf{3}}\right): \boldsymbol{\delta} 24.68\left(\mathrm{NCCH}_{2}\right), 34.06\left(\mathrm{CH}_{2} \mathrm{CH}_{2} \mathrm{CH}\right), 48.96$ $\left(\mathrm{NCH}_{2}\right), 52.62$ (d, $\left.J=4.6 \mathrm{~Hz}, 2 \times \mathrm{OCH}_{3}\right), 102.77$ (d, $\left.J=234.2 \mathrm{~Hz}, \mathrm{PC}\right), 116.00\left(\mathrm{HC}=\underline{\mathrm{CH}}_{2}\right)$, $116.14\left(\mathrm{NCH}_{2} \mathrm{CH}=\underline{\mathrm{CH}_{2}}\right), 119.79\left(\mathrm{CH}_{\text {arom }}\right), 119.83\left(\mathrm{CH}_{\text {arom }}\right), 120.84\left(\mathrm{CH}_{\text {arom }}\right), 123.25$ (d, $J=$ $12.7 \mathrm{~Hz}, \mathrm{PCC} \underline{\mathrm{C}}), 124.80$ ( $\mathrm{CH}_{\text {arom }}$ ), 132.44 (d, $J=9.2 \mathrm{~Hz}, \mathrm{NC}$ ), 132.57 (d, $J=18.5 \mathrm{~Hz}, \mathrm{PC} \underline{\mathrm{C}}$ ), $134.31\left(\mathrm{NCH}_{2} \mathrm{HC}=\mathrm{CH}_{2}\right), 137.12\left(\mathrm{HC}=\mathrm{CH}_{2}\right) .{ }^{31} \mathbf{P}-\mathbf{N M R}\left(\mathbf{1 2 1} \mathbf{~ M H z}, \mathbf{C D C l}_{\mathbf{3}}\right): \boldsymbol{\delta} 14.97 . \mathbf{I R}\left(\mathbf{c m}^{-}\right.$ 1) v vax $_{\text {max }} 1022$ (P-O), $1050(\mathrm{P}-\mathrm{O}), 1241(\mathrm{P}=\mathrm{O}), 1641(\mathrm{C}=\mathrm{C})$. MS (ESI): m/z (\%): 320.2 $\left(\mathrm{M}+\mathrm{H}^{+}, 100\right)$. Chromatography: Hex/EtOAc 55/45 $\mathrm{R}_{\mathrm{f}}=0.25$.

\section{Dimethyl 2-phenyl-2-vinyl-1,2,3,4-tetrahydropyrido[2,1-a]isoindol-6-ylphosphonate 14a}

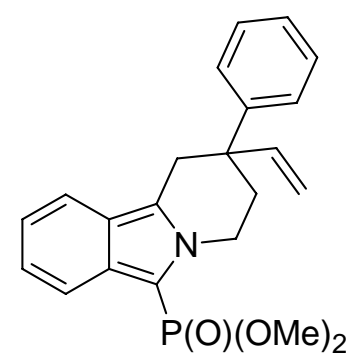

${ }^{1}$ H-NMR (300 MHz, $\mathbf{C D C l}_{3}$ ): $\boldsymbol{\delta} 2.41$ (ddd, $J=5.6 \mathrm{~Hz}, J=7.1 \mathrm{~Hz}, J=$ $13.3 \mathrm{~Hz}, 1 \mathrm{H}, \mathrm{NCH}_{2} \underline{\mathrm{C}}_{\mathrm{A}} \mathrm{H}_{\mathrm{B}}$ ), 2.57 (ddd, $J=5.3 \mathrm{~Hz}, J=7.7 \mathrm{~Hz}, J=13.3$ $\mathrm{Hz}, 1 \mathrm{H}, \mathrm{NCH}_{2} \mathrm{CH}_{\mathrm{A}} \underline{\mathrm{H}}_{\mathrm{B}}$ ), 3.49 (s, $\left.2 \mathrm{H}, \mathrm{NCC}_{2}\right), 3.64$ (d, $J=11.5 \mathrm{~Hz}, 3 \mathrm{H}$, $\mathrm{OCH}_{3}$ ), 3.66 (d, $J=11.6 \mathrm{~Hz}, 3 \mathrm{H}, \mathrm{OCH}_{3}$ ), 4.30 (ddd, $J=5.3 \mathrm{~Hz}, J=7.1$ $\mathrm{Hz}, J=14.1 \mathrm{~Hz}, 1 \mathrm{H}, \mathrm{NC}_{\mathrm{H}_{\mathrm{A}}} \mathrm{H}_{\mathrm{B}}$ ), 4.50 (ddd, $J=5.6 \mathrm{~Hz}, J=7.7 \mathrm{~Hz}, J=$ $\left.14.1 \mathrm{~Hz}, 1 \mathrm{H}, \mathrm{NCH}_{\mathrm{A}} \underline{\mathrm{H}}_{\mathrm{B}}\right), 5.01$ (d, $\left.J=17.4 \mathrm{~Hz}, 1 \mathrm{H}, \mathrm{HC}=\mathrm{C}_{\mathrm{H}} \mathrm{H}_{\mathrm{B}}\right), 5.17$ (d, $\left.J=10.7 \mathrm{~Hz}, 1 \mathrm{H}, \mathrm{HC}=\mathrm{CH}_{\mathrm{A}} \underline{\mathrm{H}}_{\mathrm{B}}\right), 6.03(\mathrm{dd}, J=10.7 \mathrm{~Hz}, J=17.4 \mathrm{~Hz}, 1 \mathrm{H}$, $\left.\underline{\mathrm{HC}}=\mathrm{CH}_{2}\right), 7.03-7.36\left(\mathrm{~m}, 7 \mathrm{H}, 7 \times \mathrm{CH}_{\text {arom }}\right), 7.61-7.65\left(\mathrm{~m}, 1 \mathrm{H}, \mathrm{CH}_{\text {arom }}\right)$, $7.89(\mathrm{~d}, J=8.4 \mathrm{~Hz}, 1 \mathrm{H}, \mathrm{PCCCCH}) .{ }^{13} \mathbf{C}-\mathbf{N M R}\left(75 \mathbf{M H z}, \mathbf{C D C l}_{3}\right): \delta 32.70\left(\mathrm{NCCH}_{2}\right), 32.81$ $\left(\mathrm{NCH}_{2} \mathrm{CH}_{2}\right), 42.24\left(\mathrm{C}_{\mathrm{q}}\right), 43.66\left(\mathrm{NCH}_{2}\right), 52.39\left(\mathrm{~d}, J=3.5 \mathrm{~Hz}, \mathrm{OCH}_{3}\right), 52.44(\mathrm{~d}, J=4.6 \mathrm{~Hz}$, 
$\left.\mathrm{OCH}_{3}\right), 101.65(\mathrm{~d}, J=235.4 \mathrm{~Hz}, \mathrm{PC}), 114.32\left(\mathrm{HC}=\underline{\mathrm{CH}}_{2}\right), 119.12\left(\mathrm{CH}_{\text {arom }}\right), 119.51\left(\mathrm{CH}_{\text {arom }}\right)$, 120.49 ( $\left.\mathrm{CH}_{\text {arom }}\right), 121.74$ (d, $J=13.9 \mathrm{~Hz}, \mathrm{PCC}$ ), 126.47 (2 x $\left.\mathrm{CH}_{\text {arom }}\right), 129.96\left(\mathrm{CH}_{\text {arom }}\right)$, 128.44 (d, $J=9.2 \mathrm{~Hz}, \mathrm{NC}), 128.73$ ( $2 \times \mathrm{CH}_{\text {arom}}$ ), 133.28 (d, $J=18.5 \mathrm{~Hz}$, PCC $), 143.47$ $\left(\mathrm{HC}=\mathrm{CH}_{2}\right), 144.08\left(\mathrm{C}_{\mathrm{q}}, \mathrm{Ph}\right) .{ }^{31} \mathbf{P}-\mathbf{N M R}\left(\mathbf{1 2 1} \mathbf{M H z}, \mathbf{C D C l}_{3}\right): \boldsymbol{\delta} 15.29 . \mathbf{I R}\left(\mathbf{c m}^{-1}\right) \mathbf{v}_{\max }$ : $1021(\mathrm{P}-$ O), 1047 (P-O), $1243(\mathrm{P}=\mathrm{O}), 1623$ (C=C). MS (ESI): m/z (\%): $382.3\left(\mathrm{M}+\mathrm{H}^{+}, 100\right)$. Chromatography: Hex/EtOAc $1 / 1 \mathrm{R}_{\mathrm{f}}=0.14$. Yield: $68 \%$.

\section{Dimethyl 2-(4-fluorophenyl)-2-vinyl-1,2,3,4-tetrahydropyrido[2,1-a]isoindol-6-} ylphosphonate 14b

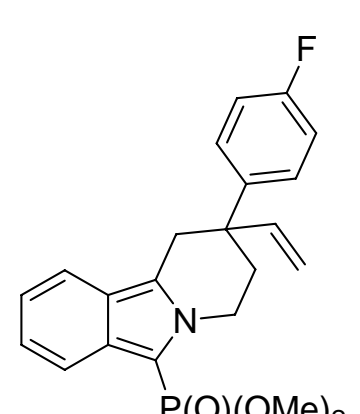

${ }^{1}$ H-NMR (300 MHz, $\left.\mathbf{C D C l}_{3}\right): \boldsymbol{\delta} 2.38(\mathrm{dt}, J=6.3 \mathrm{~Hz}, J=13.8 \mathrm{~Hz}, 1 \mathrm{H}$, $\mathrm{NCH}_{2} \underline{\mathrm{C}}_{\mathrm{A}} \mathrm{H}_{\mathrm{B}}$ ), 2.52 (dt, $J=6.3 \mathrm{~Hz}, J=13.8 \mathrm{~Hz}, 1 \mathrm{H}, \mathrm{NCH}_{2} \mathrm{CH}_{\mathrm{A}} \underline{\mathrm{H}}_{\mathrm{B}}$ ), 3.45 (s, $2 \mathrm{H}, \mathrm{NCC} \underline{\mathrm{H}}_{2}$ ), 3.65 (d, $J=11.5 \mathrm{~Hz}, 3 \mathrm{H}, \mathrm{OCH}_{3}$ ), 3.67 (d, $J=11.5$ $\mathrm{Hz}, 3 \mathrm{H}, \mathrm{OCH}_{3}$ ), 4.27 (dt, $\left.J=6.3 \mathrm{~Hz}, J=14.7 \mathrm{~Hz}, 1 \mathrm{H}, \mathrm{NC}_{\mathrm{H}_{\mathrm{A}}} \mathrm{H}_{\mathrm{B}}\right), 4.51$ (dt, $\left.J=6.3 \mathrm{~Hz}, J=14.7 \mathrm{~Hz}, 1 \mathrm{H}, \mathrm{NCH}_{\mathrm{A}} \underline{\mathrm{H}}_{\mathrm{B}}\right), 4.99$ (d, $J=17.6 \mathrm{~Hz}, 1 \mathrm{H}$, $\left.\mathrm{HC}=\mathrm{C}_{\mathrm{H}} \mathrm{H}_{\mathrm{B}}\right), 5.17\left(\mathrm{~d}, J=10.7 \mathrm{~Hz}, 1 \mathrm{H}, \mathrm{HC}=\mathrm{CH}_{\mathrm{A}} \underline{\mathrm{H}}_{\mathrm{B}}\right), 5.99$ (dd, $J=10.7$ $\left.\mathrm{Hz}, J=17.6 \mathrm{~Hz}, 1 \mathrm{H}, \underline{\mathrm{HC}}=\mathrm{CH}_{2}\right), 6.93-7.26$ (m, $\left.6 \mathrm{H}, 6 \times \mathrm{CH}_{\text {arom }}\right), 7.62$ (dt, $\left.J=0.9 \mathrm{~Hz}, J=8.5 \mathrm{~Hz}, 1 \mathrm{H}, \mathrm{CH}_{\text {arom }}\right), 7.89$ (dt, $J=0.9 \mathrm{~Hz}, J=8.5 \mathrm{~Hz}, 1 \mathrm{H}$, $\mathrm{P}(\mathrm{O})(\mathrm{OMe})_{2}$ PCCCC $\left.\underline{\mathrm{H}}\right) .{ }^{13} \mathbf{C}-\mathrm{NMR}\left(\mathbf{7 5} \mathbf{~ M H z}, \mathbf{C D C l}_{3}\right): \boldsymbol{\delta} 32.85\left(\mathrm{NCCH}_{2}\right), 32.94$ $\left(\mathrm{NCH}_{2} \mathrm{CH}_{2}\right), 41.87\left(\mathrm{C}_{\mathrm{q}}\right), 43.60\left(\mathrm{NCH}_{2}\right), 52.39\left(\mathrm{~d}, J=4.6 \mathrm{~Hz}, \mathrm{OCH}_{3}\right), 52.42(\mathrm{~d}, J=4.6 \mathrm{~Hz}$, $\left.\mathrm{OCH}_{3}\right), 101.84(\mathrm{~d}, J=236.5 \mathrm{~Hz}, \mathrm{PC}), 114.46\left(\mathrm{HC}=\mathrm{CH}_{2}\right), 115.47$ (d, $\left.J=21.9 \mathrm{~Hz}, 2 \times \mathrm{CH}_{\text {arom }}\right)$, 119.05 ( $\left.\mathrm{CH}_{\text {arom }}\right), 119.51$ ( $\left.\mathrm{CH}_{\text {arom }}\right), 120.58\left(\mathrm{CH}_{\text {arom }}\right), 121.73$ (d, $\left.J=12.7 \mathrm{~Hz}, \mathrm{PCC} \underline{\mathrm{C}}\right), 125.13$ ( $\mathrm{CH}_{\text {arom }}$ ), 128.09 (d, $\left.J=11.5 \mathrm{~Hz}, \mathrm{NC}\right), 128.22$ (d, $J=8.1 \mathrm{~Hz}, 2 \times \mathrm{CH}_{\text {arom }}$ ), 133.24 (d, $J=18.5$ $\mathrm{Hz}, \mathrm{PC} \underline{\mathrm{C}}), 139.77$ (d, $\left.J=3.5 \mathrm{~Hz}, \mathrm{C}_{\mathrm{q}, \text { arom }}\right), 143.38\left(\mathrm{HC}=\mathrm{CH}_{2}\right), 161.64(\mathrm{~d}, J=246.9 \mathrm{~Hz}$, $\left.\mathrm{FC}_{\mathrm{q}, \text { arom }}\right) .{ }^{31} \mathrm{P}-\mathrm{NMR}\left(121 \mathrm{MHz}, \mathrm{CDCl}_{3}\right): \delta$ 15.17. ${ }^{\mathbf{1 9}} \mathrm{F}-\mathrm{NMR}\left(282 \mathrm{MHz}, \mathbf{C D C l}_{3}\right): \delta$-115.68 -

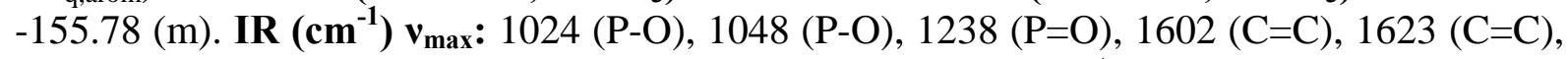
$1636(\mathrm{C}=\mathrm{C}), 1702(\mathrm{C}=\mathrm{C})$. MS (ESI): m/z (\%): $400.2\left(\mathrm{M}+\mathrm{H}^{+}, 100\right)$. Chromatography: Hex/EtOAc 4/6 $\mathrm{R}_{\mathrm{f}}=0.17$. Yield: $82 \%$.

\section{Synthesis of isoindole 13.}

In a dry flask, 2-ethynylbenzaldehyde $(3.84 \mathrm{mmol})$ is dissolved into diethylether $(6 \mathrm{~mL}$, freshly distilled from Na-metal). To this solution is added $\mathrm{LiClO}_{4}$ (7.5 equiv, $28.8 \mathrm{mmol}$, dried for $24 \mathrm{~h}$ at $110^{\circ} \mathrm{C}$ ). This mixture is stirred for 5 minutes. Subsequently pyrrolidine is added (2 equiv, $7.69 \mathrm{mmol}$, dissolved in $1 \mathrm{~mL}$ dry diethylether). This mixture is stirred for 20 minutes after which $\mathrm{P}(\mathrm{OMe})_{3}$ is added (1.5 equiv, $\left.5.76 \mathrm{mmol}\right)$. The reaction is stirred for 4 hours after which $\mathrm{HCl}(3 \mathrm{~N}, 20 \mathrm{~mL})$ is very carefully added. The mixture is extracted with $\mathrm{CH}_{2} \mathrm{Cl}_{2}(3 \times 20 \mathrm{~mL})$ and dried using $\mathrm{MgSO}_{4}$. After filtration of the solids and removal of the volatiles, the obtained compound was purified using column chromatography.

\section{Dimethyl 2-(4-chlorobutyl)-3-methyl-2H-isoindol-1-ylphosphonate 13}

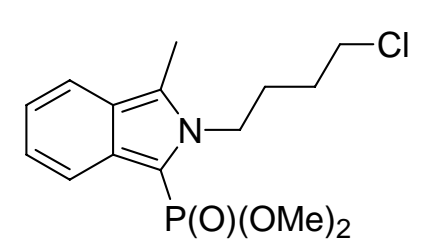

${ }^{1} \mathbf{H}-\mathrm{NMR}\left(300 \mathrm{MHz}, \mathbf{C D C l}_{3}\right)$ : $\boldsymbol{\delta}$ 1.88-2.02 (m, 4H, $\left.\mathrm{NCH}_{2} \mathrm{CH}_{2} \mathrm{CH}_{2}\right)$, 2.61 (s, $\left.3 \mathrm{H}, \mathrm{CH}_{3}\right), 3.59\left(\mathrm{t}, J=6.2 \mathrm{~Hz}, 2 \mathrm{H}, \mathrm{C}_{2} \mathrm{Cl}\right), 3.73(\mathrm{~d}, J=11.3$ $\left.\mathrm{Hz}, 6 \mathrm{H}, 2 \times \mathrm{OCH}_{3}\right), 4.55$ (t, $\left.J=7.6 \mathrm{~Hz}, 2 \mathrm{H}, \mathrm{NC}_{2}\right), 7.02$ (t, $J=8.0$ $\left.\mathrm{Hz}, 1 \mathrm{H}, \mathrm{CH}_{\text {arom }}\right), 7.17$ (t, $\left.J=8.0 \mathrm{~Hz}, 1 \mathrm{H}, \mathrm{CH}_{\text {arom }}\right), 7.56$ (d, $J=8.0$ $\mathrm{Hz}, 1 \mathrm{H}, \mathrm{PCCC} \underline{\mathrm{H}}), 7.80$ (d, $J=8.0 \mathrm{~Hz}, 1 \mathrm{H}, \mathrm{PCCCC} \underline{\mathrm{H}}) .{ }^{13} \mathbf{C}-\mathbf{N M R}(\mathbf{7 5}$ 
MHz, $\left.\mathbf{C D C l}_{3}\right): \boldsymbol{\delta} 10.41\left(\mathrm{CH}_{3}\right), 28.96\left(\mathrm{CH}_{2}\right), 29.75\left(\mathrm{CH}_{2}\right), 44.53\left(\mathrm{ClCH}_{2}\right), 46.21\left(\mathrm{NCH}_{2}\right)$, 52.61 (d, $\left.J=4.6 \mathrm{~Hz}, 2 \times \mathrm{OCH}_{3}\right), 102.16$ (d, $\left.J=235.4 \mathrm{~Hz}, \mathrm{PC}\right), 119.47\left(\mathrm{CH}_{\text {arom }}\right), 119.67$ ( $\left.\mathrm{CH}_{\text {arom }}\right), 120.67\left(\mathrm{CH}_{\text {arom }}\right), 123.51$ (d, $\left.J=12.7 \mathrm{~Hz}, \mathrm{PCCC}\right), 124.89\left(\mathrm{CH}_{\text {arom }}\right), 128.44$ (d, $J=$ $10.4 \mathrm{~Hz}, \mathrm{NC}), 132.35$ (d, $J=17.3 \mathrm{~Hz}$, PCC). ${ }^{31} \mathbf{P}-\mathbf{N M R}\left(\mathbf{1 2 1} \mathbf{M H z}, \mathbf{C D C l}_{\mathbf{3}}\right): \boldsymbol{\delta}$ 15.16. IR (cm 1) v max $_{\text {ma }} 1024$ (P-O), $1048(\mathrm{P}-\mathrm{O}), 1245(\mathrm{P}=\mathrm{O}), 1710(\mathrm{C}=\mathrm{C})$. MS (ESI): m/z (\%): 330.2/332.3 $\left(\mathrm{M}+\mathrm{H}^{+}, 100\right)$. Chromatography: EtOAc $\mathrm{R}_{\mathrm{f}}=0.59$. Yield: $8 \%$.

Synthesis of azepino isoindole 16: Compound 4i (0.2 g, $0.63 \mathrm{mmol})$ was dissolved in benzene $(20 \mathrm{~mL})$ and the second generation Grubbs' catalyst (0.02 equiv, $0.011 \mathrm{~g}, 0.013$ mmol) was added. The reaction was allowed to reflux for $16 \mathrm{~h}$ under a $\mathrm{N}_{2}$-atmosphere. The product was coated on silica gel by removal of the solvent in vacuo and purified by flash chromatography

\section{Dimethyl 10,11-dihydro-7H-azepino[2,1-a]isoindol-5-ylphosphonate 16}

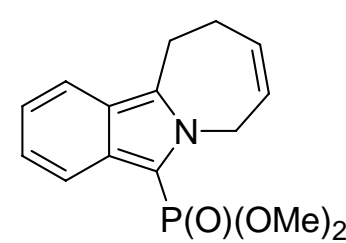

${ }^{1}$ H-NMR (300 MHz, $\mathbf{C D C l}_{3}$ ): $\boldsymbol{\delta}$ 2.42-2.48 (m, 2H, $\left.\mathrm{CH}_{2} \underline{\mathrm{C}}_{2} \mathrm{CH}\right), 3.38$ (t, $J=6.0 \mathrm{~Hz}, 2 \mathrm{H}, \mathrm{C}_{2} \mathrm{CH}_{2} \mathrm{CH}$ ), $3.72\left(\mathrm{~d}, J=11.5 \mathrm{~Hz}, 6 \mathrm{H}, 2 \times \mathrm{OCH}_{3}\right)$, 5.24-5.28 (m, 2H, $\mathrm{NCH}_{2}$ ), 5.75-5.94 (m, 2H, $\left.\underline{\mathrm{HC}}=\mathrm{C} \underline{\mathrm{H}}\right), 7.03$ (ddd, $J=$ $\left.1.0 \mathrm{~Hz}, J=6.6 \mathrm{~Hz}, J=8.4 \mathrm{~Hz}, 1 \mathrm{H}, \mathrm{CH}_{\text {arom }}\right), 7.16$ (ddd, $J=1.0 \mathrm{~Hz}, J=$ $\left.6.6 \mathrm{~Hz}, J=8.7 \mathrm{~Hz}, 1 \mathrm{H}, \mathrm{CH}_{\text {arom }}\right), 7.58$ (ddt, $J=1.0 \mathrm{~Hz}, J=2.2 \mathrm{~Hz}, J=$ $8.4 \mathrm{~Hz}, 1 \mathrm{H}, \mathrm{PCCC} \underline{\mathrm{H}}), 7.90(\mathrm{dt}, J=1.0 \mathrm{~Hz}, J=8.7 \mathrm{~Hz}, 1 \mathrm{H}$, PCCCC $\underline{H})$. ${ }^{13}$ C-NMR (75 MHz, CDCl 3 ): $\boldsymbol{\delta} 21.86\left(\mathrm{NCCH}_{2}\right), 28.13\left(\mathrm{CH}_{2} \mathrm{CH}_{2} \mathrm{CH}\right), 43.98\left(\mathrm{NCH}_{2}\right), 52.59$ (d, $\left.J=4.6 \mathrm{~Hz}, 2 \times \mathrm{OCH}_{3}\right), 102.46$ (d, $\left.J=234.2 \mathrm{~Hz}, \mathrm{PC}\right), 119.08\left(\mathrm{CH}_{\text {arom }}\right), 119.75\left(\mathrm{CH}_{\text {arom }}\right)$, $120.72\left(\mathrm{CH}_{\text {arom }}\right), 122.26$ (d, $\left.J=12.7 \mathrm{~Hz}, \mathrm{PCC} \underline{)}\right), 122.62(\mathrm{HC}=\mathrm{CH}), 124.44\left(\mathrm{CH}_{\text {arom }}\right), 132.09$ (d, $J=17.3 \mathrm{~Hz}, \mathrm{PCC}), 133.15$ ( $\left.\mathrm{NCH}_{2} \underline{\mathrm{CH}}\right), 133.87$ (d, $\left.J=9.2 \mathrm{~Hz}, \mathrm{NC}\right) .{ }^{31} \mathbf{P}-\mathbf{N M R}(121 \mathrm{MHz}$, $\left.\mathbf{C D C l}_{3}\right): \boldsymbol{\delta}$ 15.26. IR (cm $\left.{ }^{-1}\right) \mathbf{v}_{\text {max }}$ : $1025(\mathrm{P}-\mathrm{O}), 1047(\mathrm{P}-\mathrm{O}), 1224(\mathrm{P}=\mathrm{O}), 1694(\mathrm{C}=\mathrm{C}) . \mathbf{M S}$ (ESI): m/z (\%): $292.3\left(\mathrm{M}+\mathrm{H}^{+}, 100\right)$. Chromatography: Hex/EtOAc 4/6 $\mathrm{R}_{\mathrm{f}}=0.20$. Yield: 95\%. 
N. Dieltiens and C. V. Stevens

2a ${ }^{13} \mathrm{C}-$ Spectrum

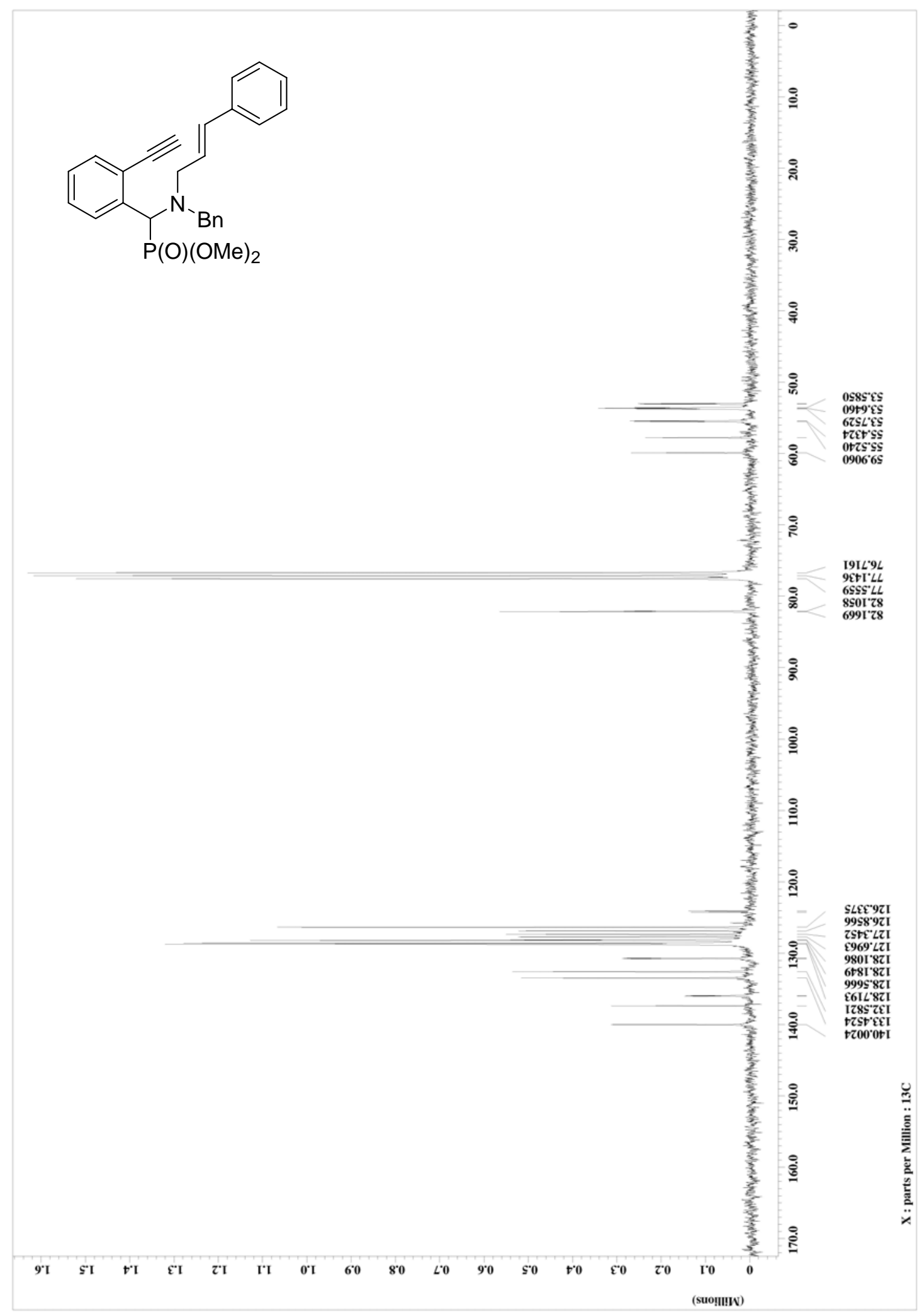


N. Dieltiens and C. V. Stevens

2a ${ }^{1} \mathrm{H}$-Spectrum

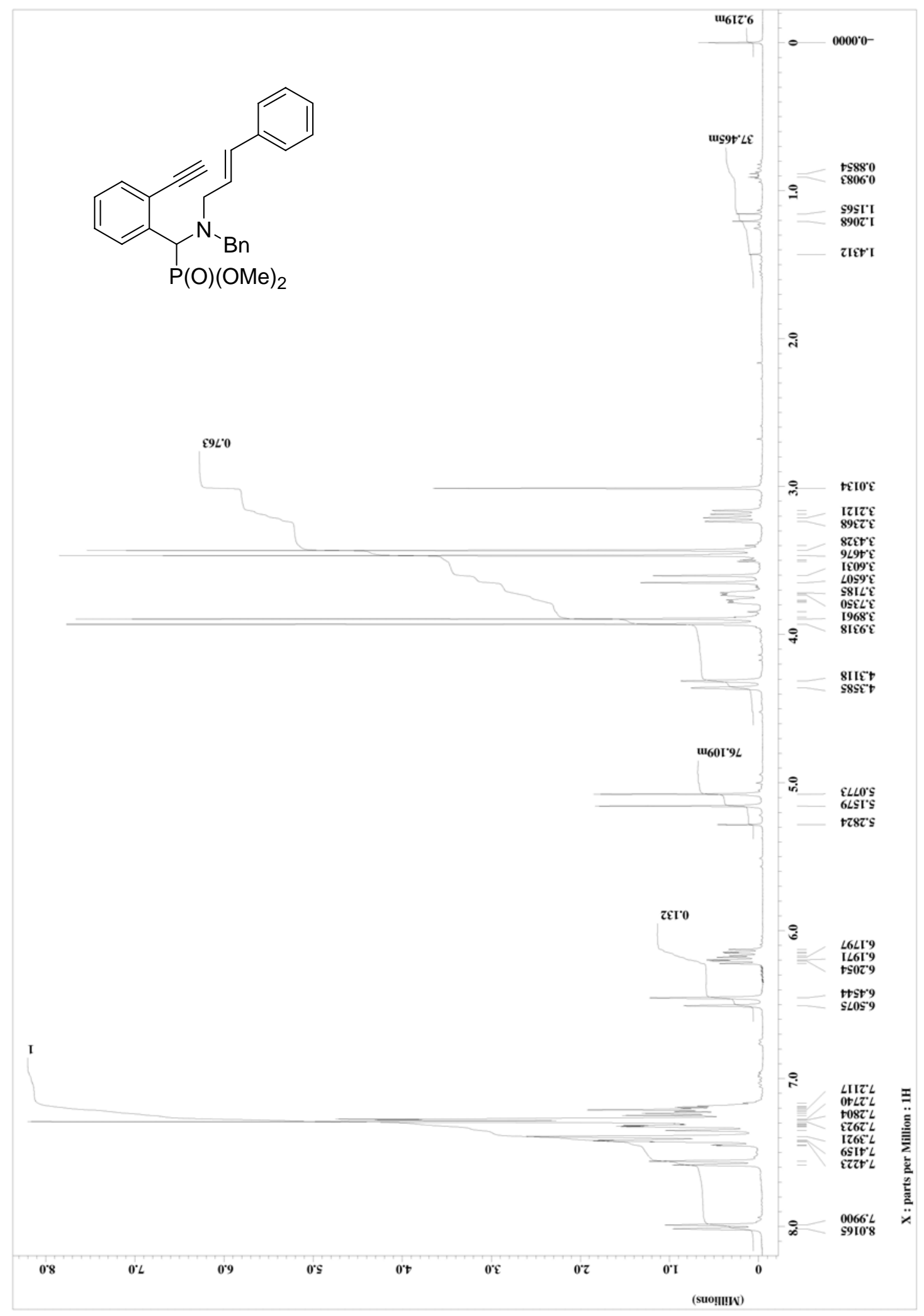


N. Dieltiens and C. V. Stevens

2b ${ }^{13} \mathrm{C}$-Spectrum

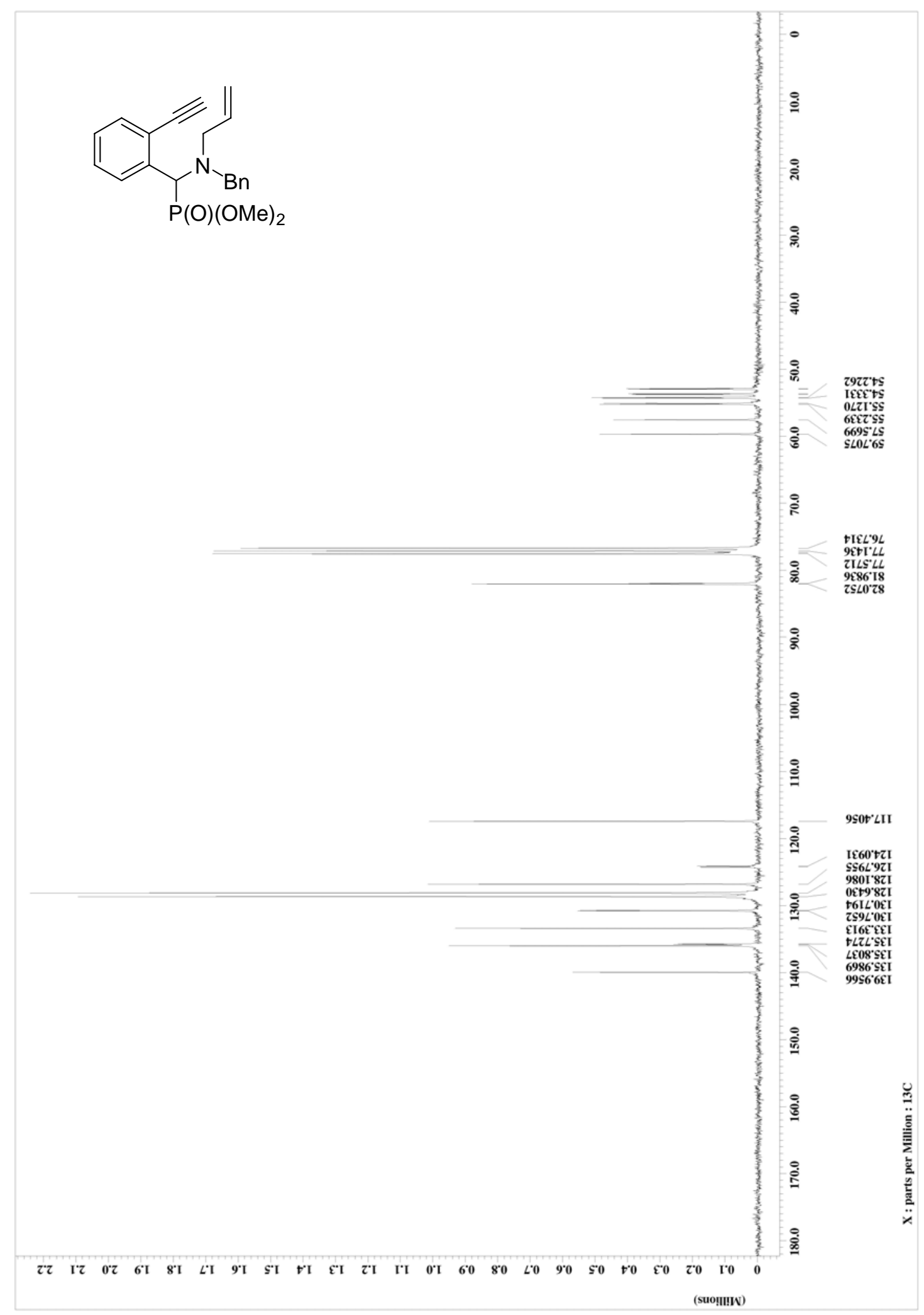


N. Dieltiens and C. V. Stevens

2b ${ }^{1} \mathrm{H}$-Spectrum

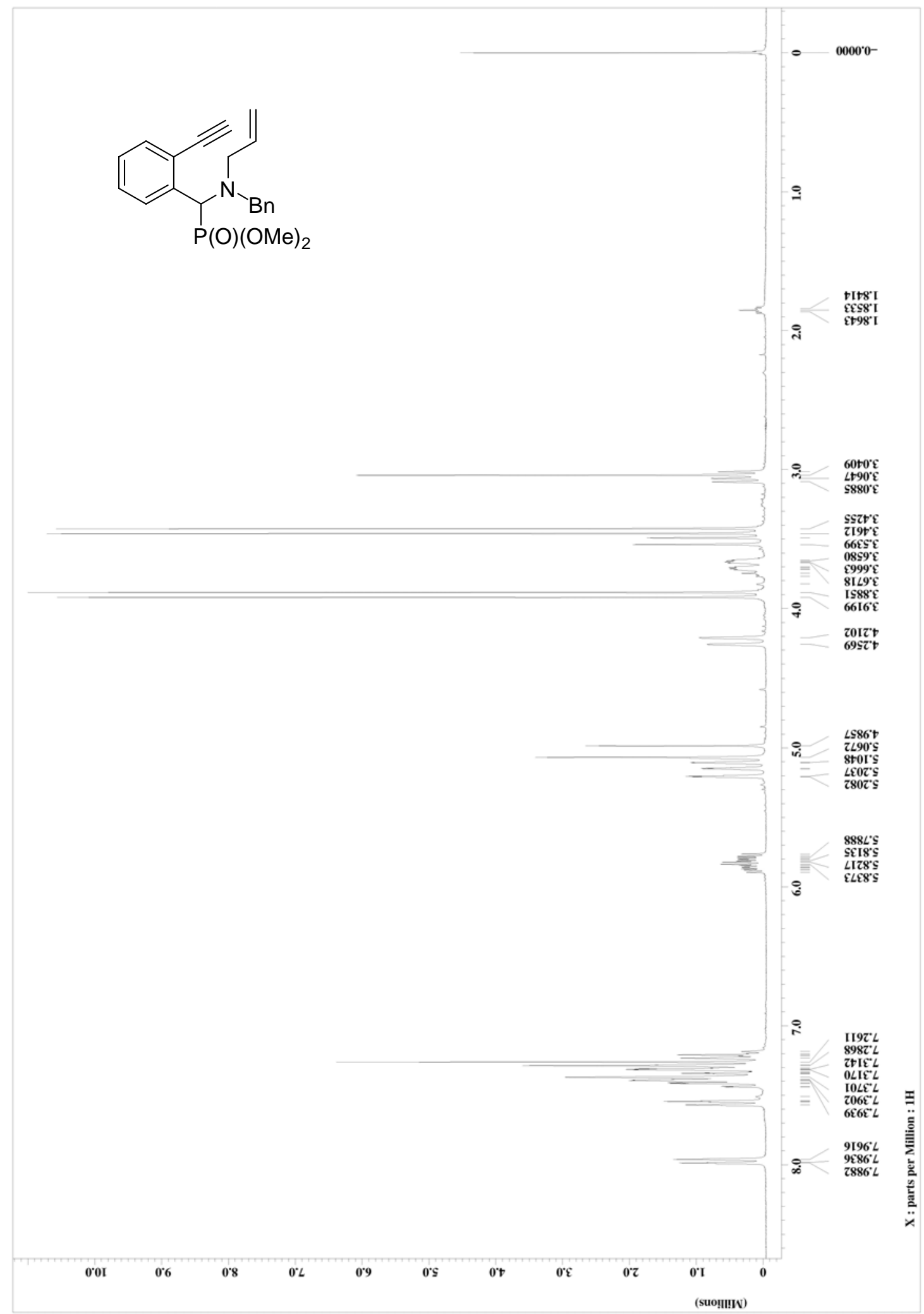


N. Dieltiens and C. V. Stevens

2c ${ }^{13} \mathrm{C}$-Spectrum

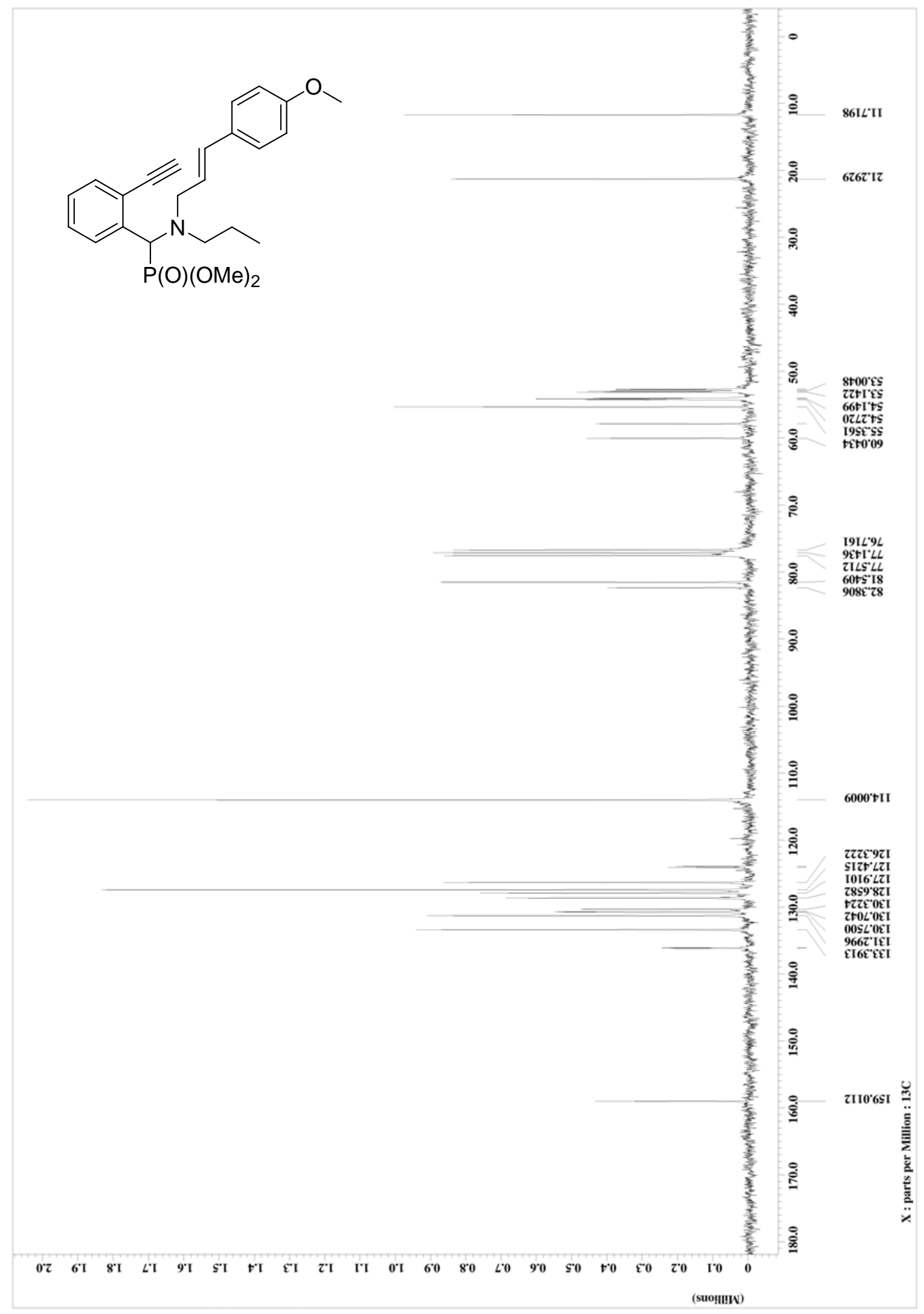


N. Dieltiens and C. V. Stevens

\section{2c ${ }^{1} \mathrm{H}$-Spectrum}

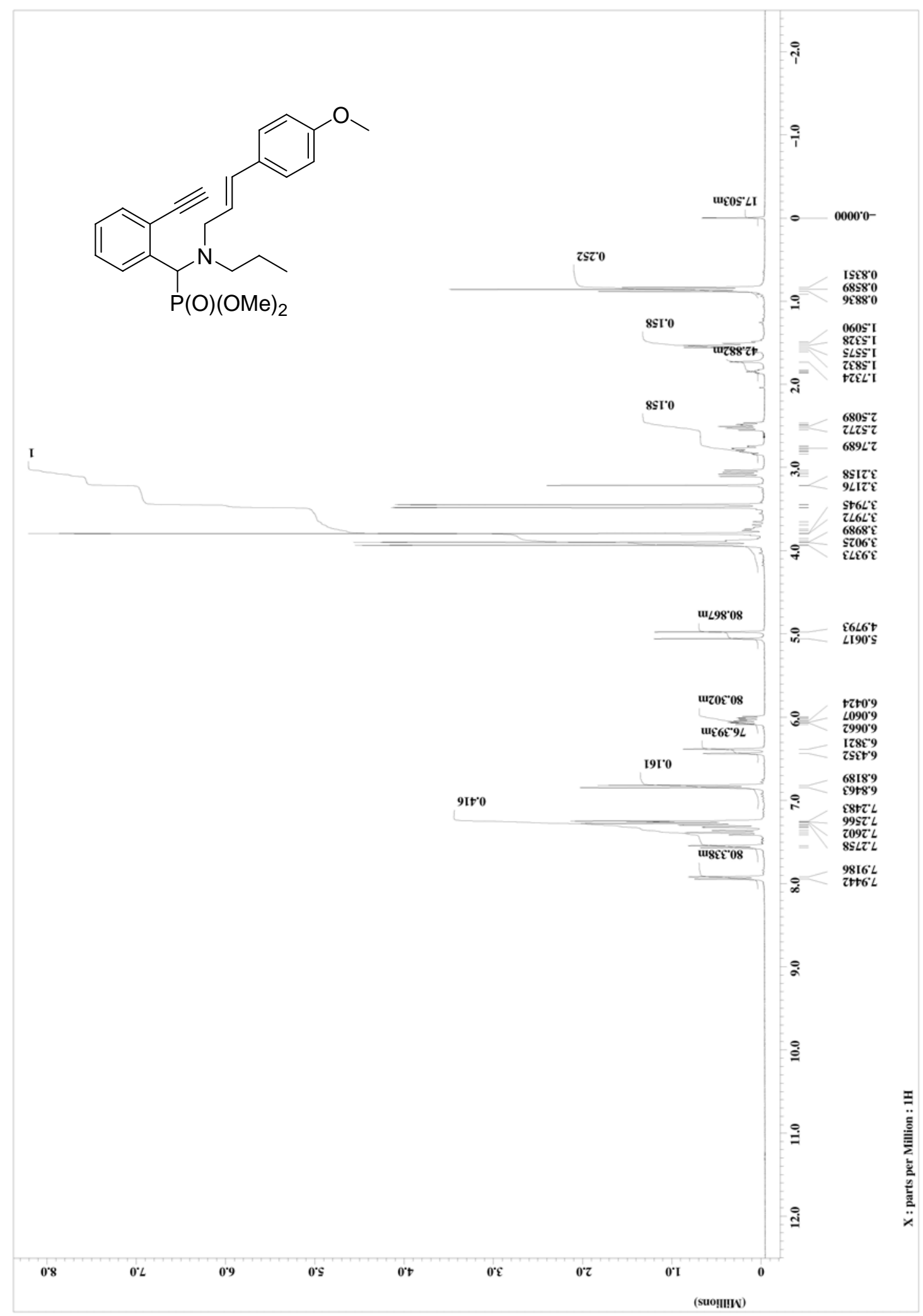


N. Dieltiens and C. V. Stevens

2d ${ }^{13}$ C-Spectrum

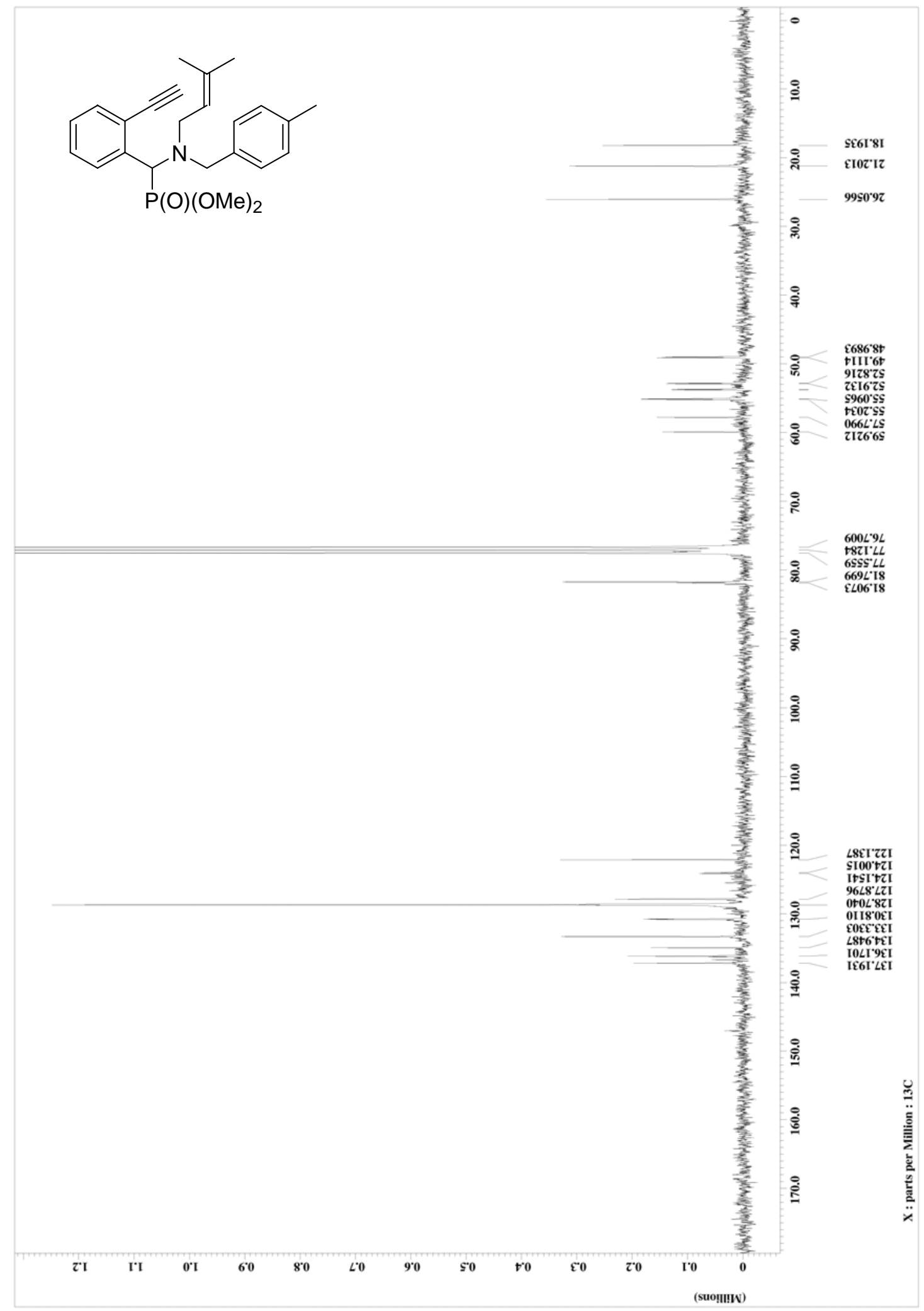


N. Dieltiens and C. V. Stevens

2d ${ }^{1} \mathrm{H}$-Spectrum

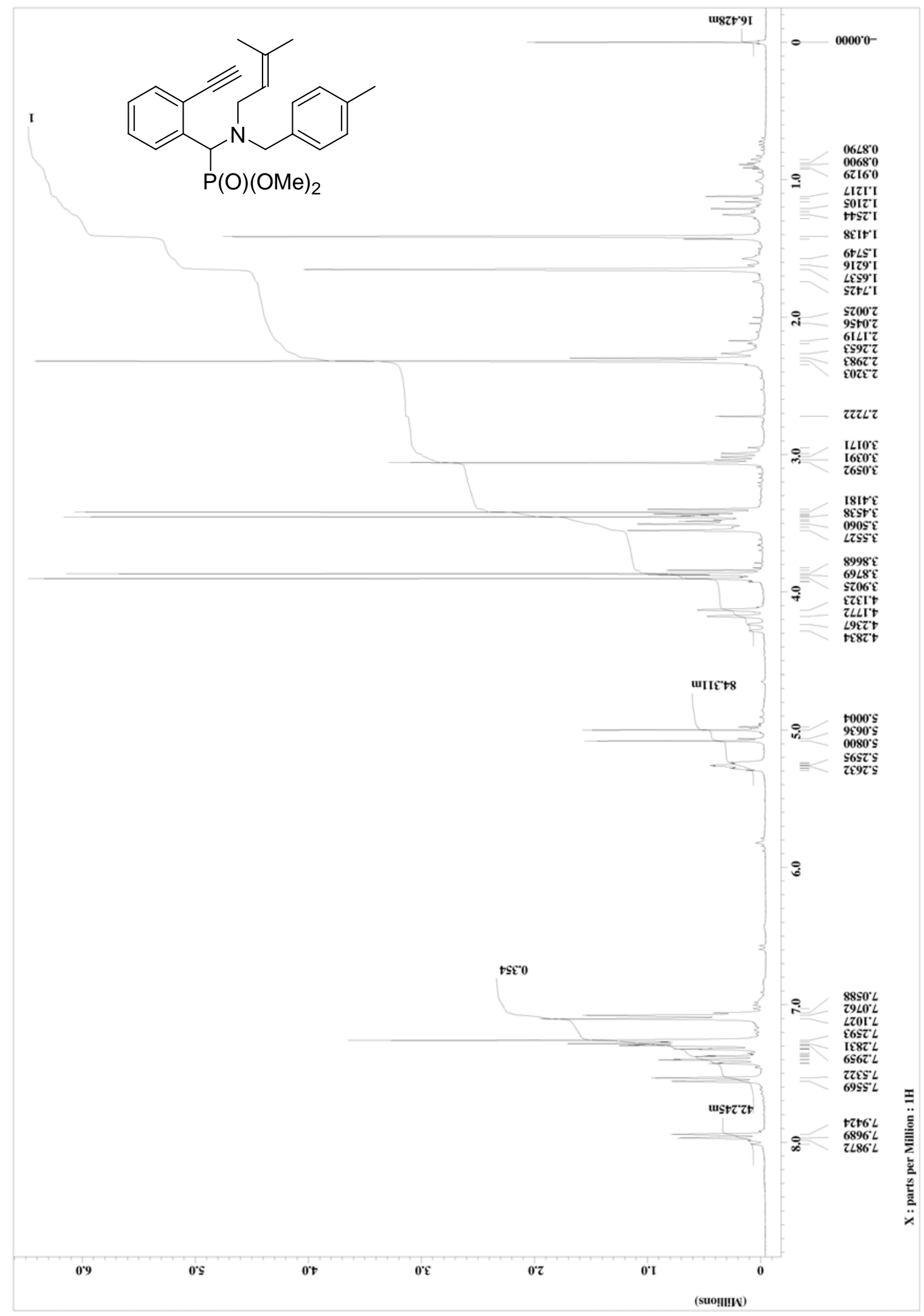


N. Dieltiens and C. V. Stevens

2e ${ }^{13}$ C-Spectrum
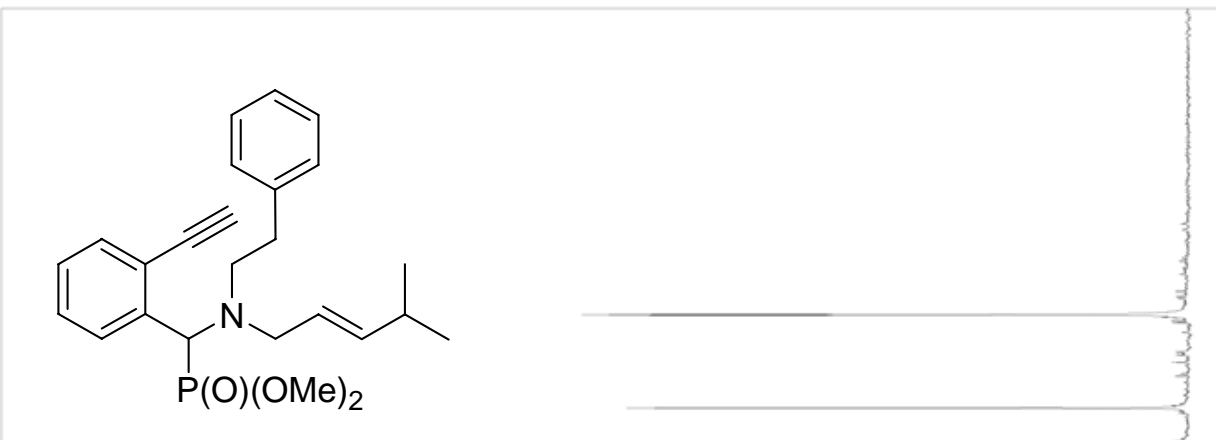

$\stackrel{\leftrightarrow}{\leftrightarrows}$

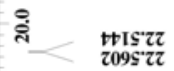

용 $28860 \varepsilon$

ostz't

棉

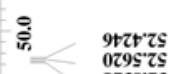

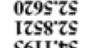

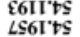

OSzt'8s
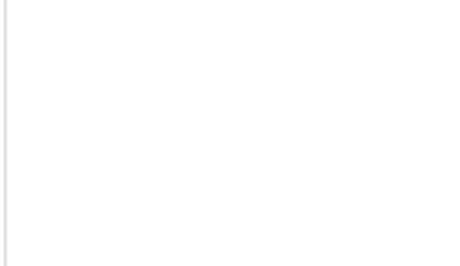

0 's

ot

${ }^{*} \varepsilon$

o'z

O'I

0ะz8.9L

SOSZ LL
$8299^{\circ} \angle L$

EL06. T8

$\angle t+078$

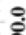

蓄

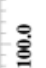

$\stackrel{8}{\Xi}$

ڤั

I08L'tzI

$6 \subseteq 96 \angle \mathrm{LI}$

عI9z:8zI

t08L'8ZI

It66 8zI

$0 z 850 \varepsilon 1$
$8 \angle 79^{\circ} 0$ \&

$8 \angle Z 9^{\circ} 0 \varepsilon I$
I $\angle \varepsilon t^{\circ} \varepsilon \varepsilon I$

6SI ${ }^{\circ} 9 \varepsilon \mathrm{I}$

$89 \varepsilon 50 t$

ats

(suo!n!!) 
N. Dieltiens and C. V. Stevens

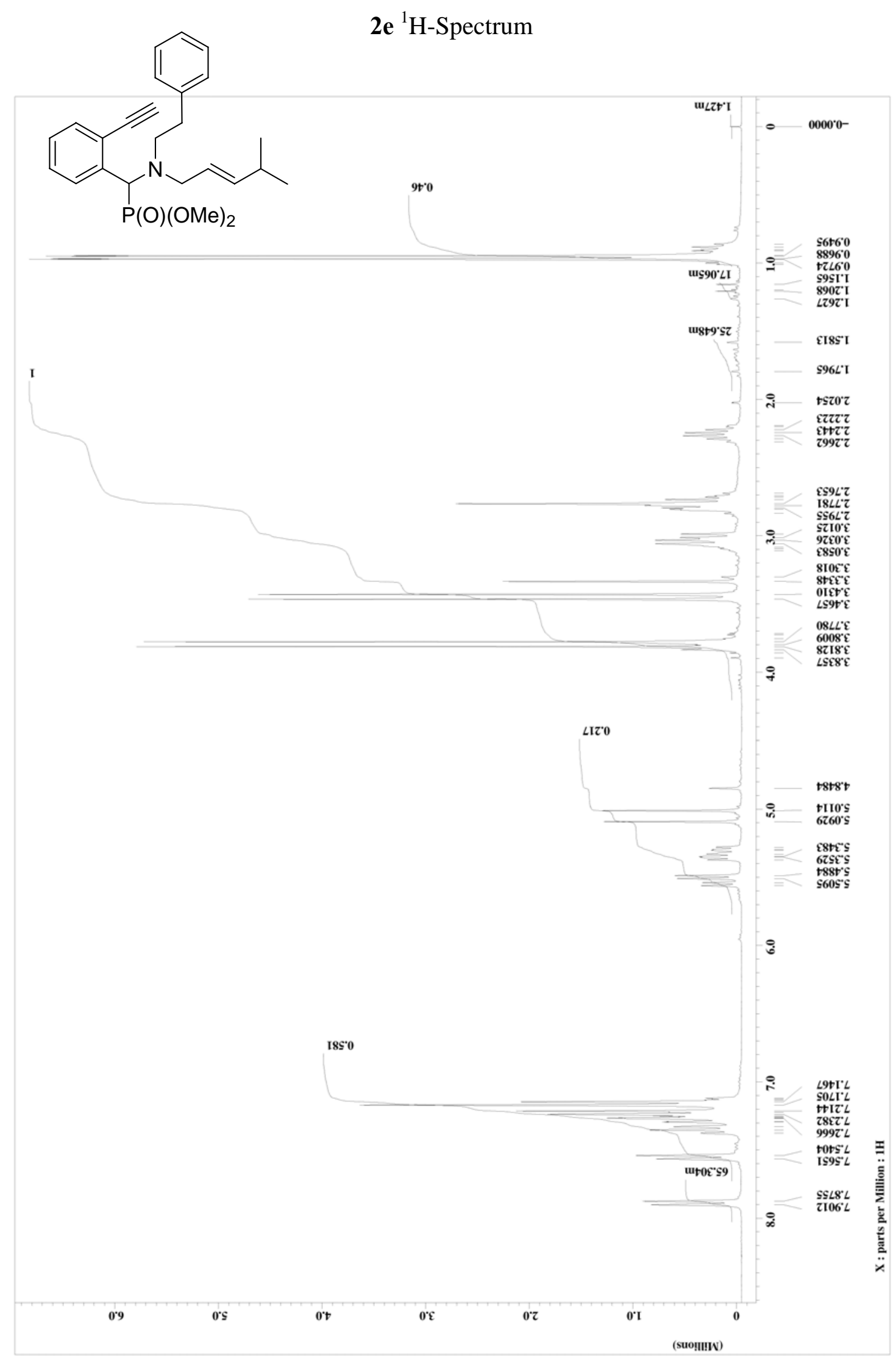


N. Dieltiens and C. V. Stevens

2f ${ }^{13} \mathrm{C}$-Spectrum

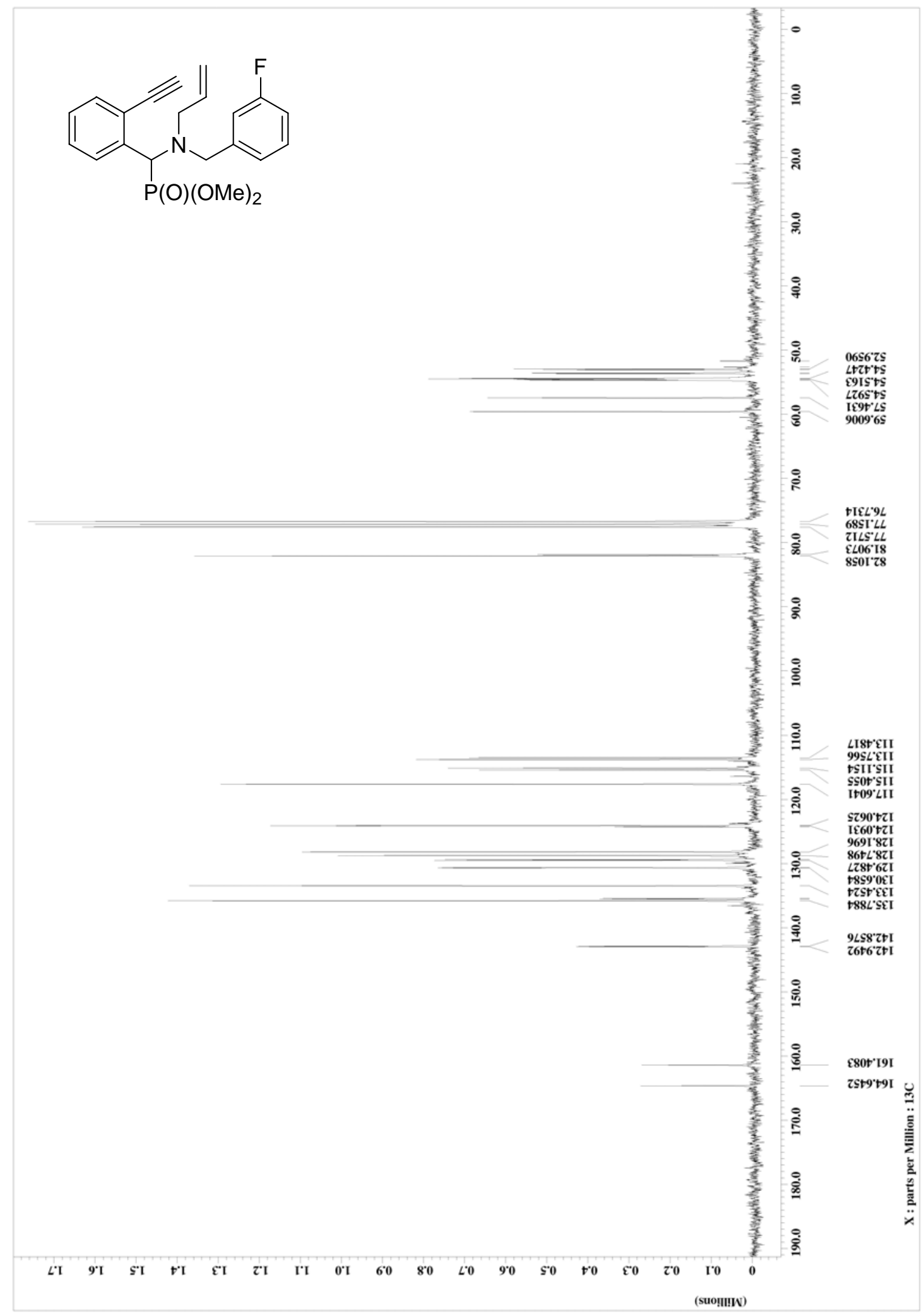


N. Dieltiens and C. V. Stevens

\section{$2 \mathbf{f}^{1} \mathrm{H}$-Spectrum}

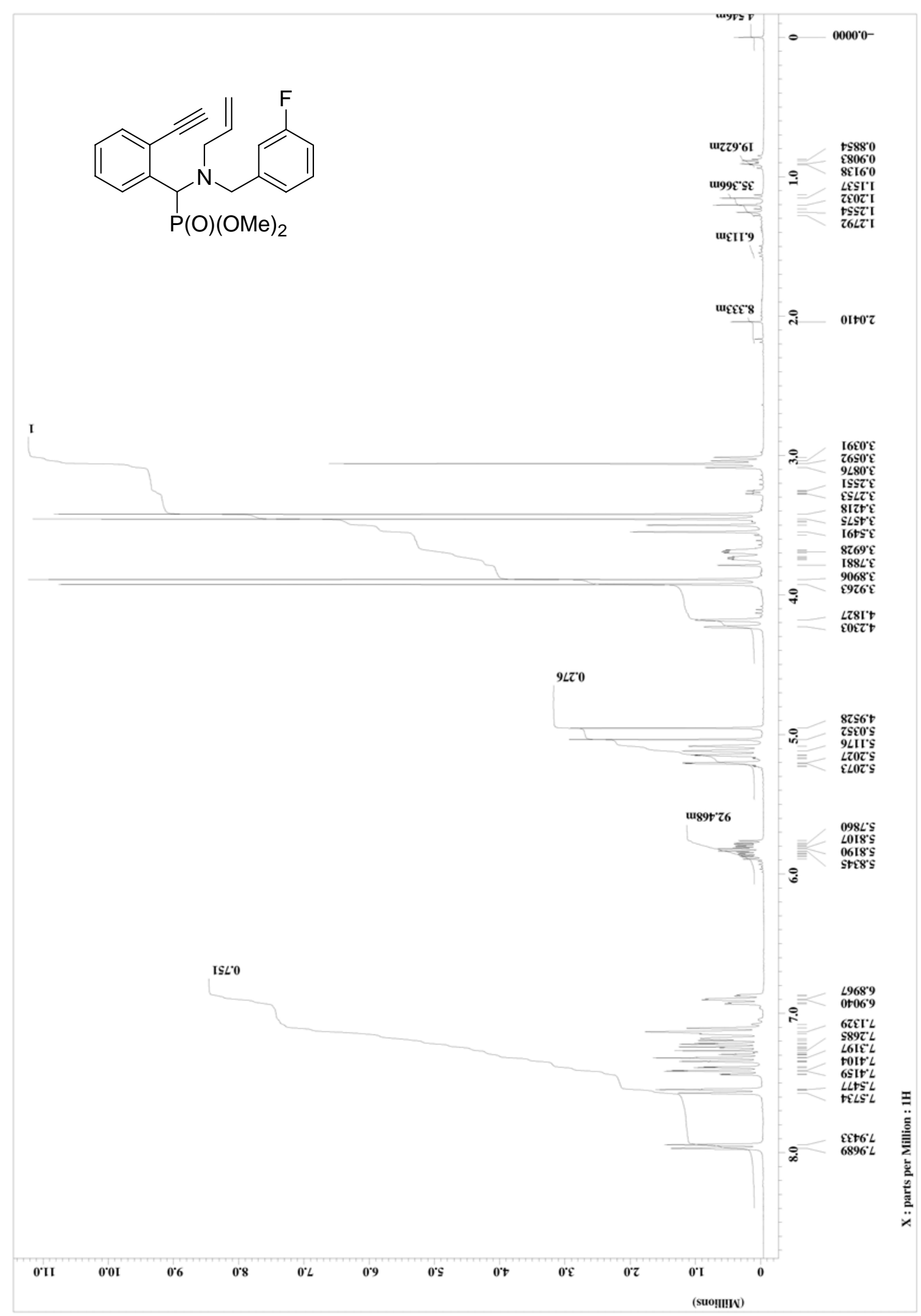


N. Dieltiens and C. V. Stevens

$\mathbf{2 g}{ }^{13} \mathrm{C}-$ Spectrum

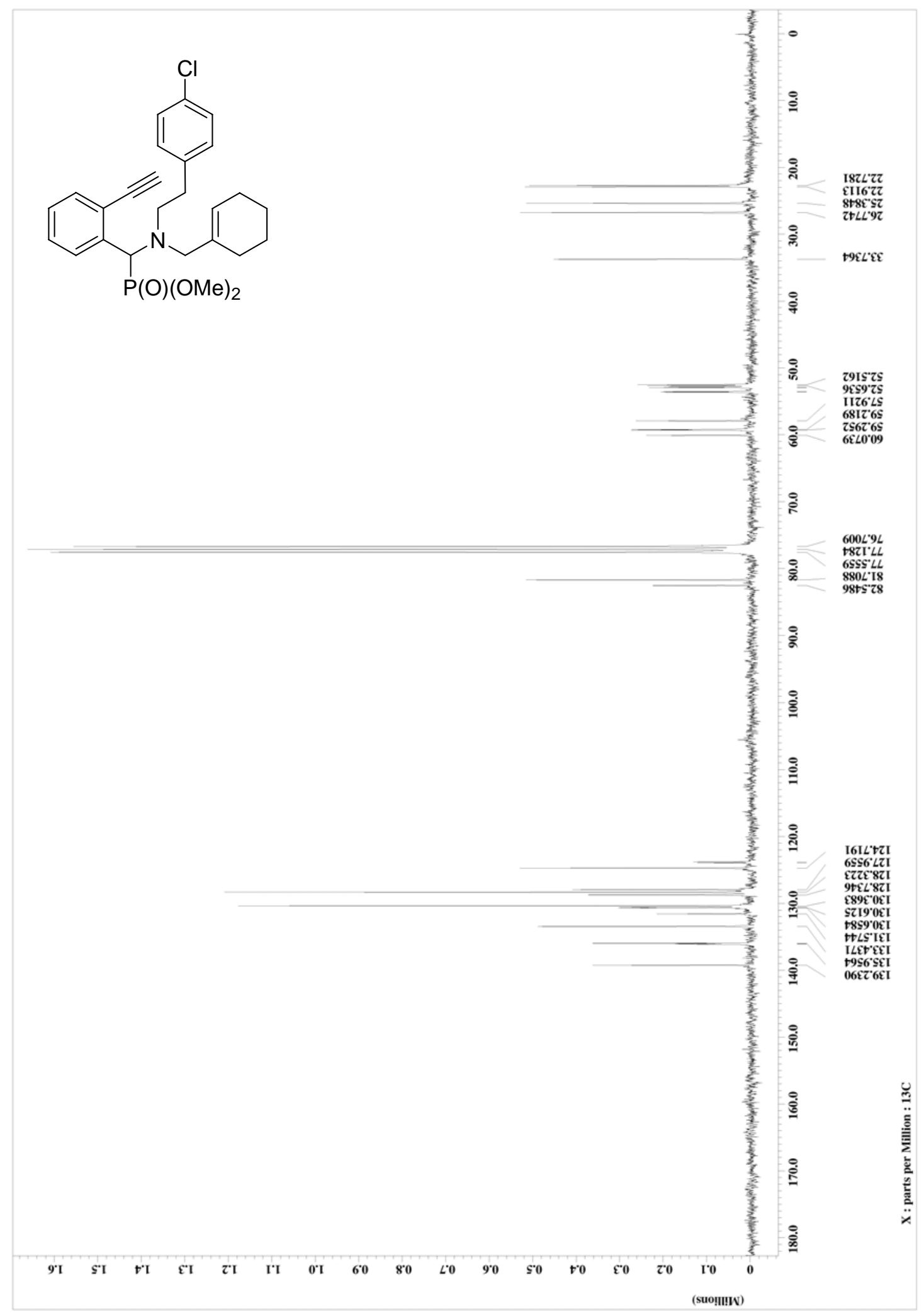


N. Dieltiens and C. V. Stevens

2g ${ }^{1} \mathrm{H}-$ Spectrum

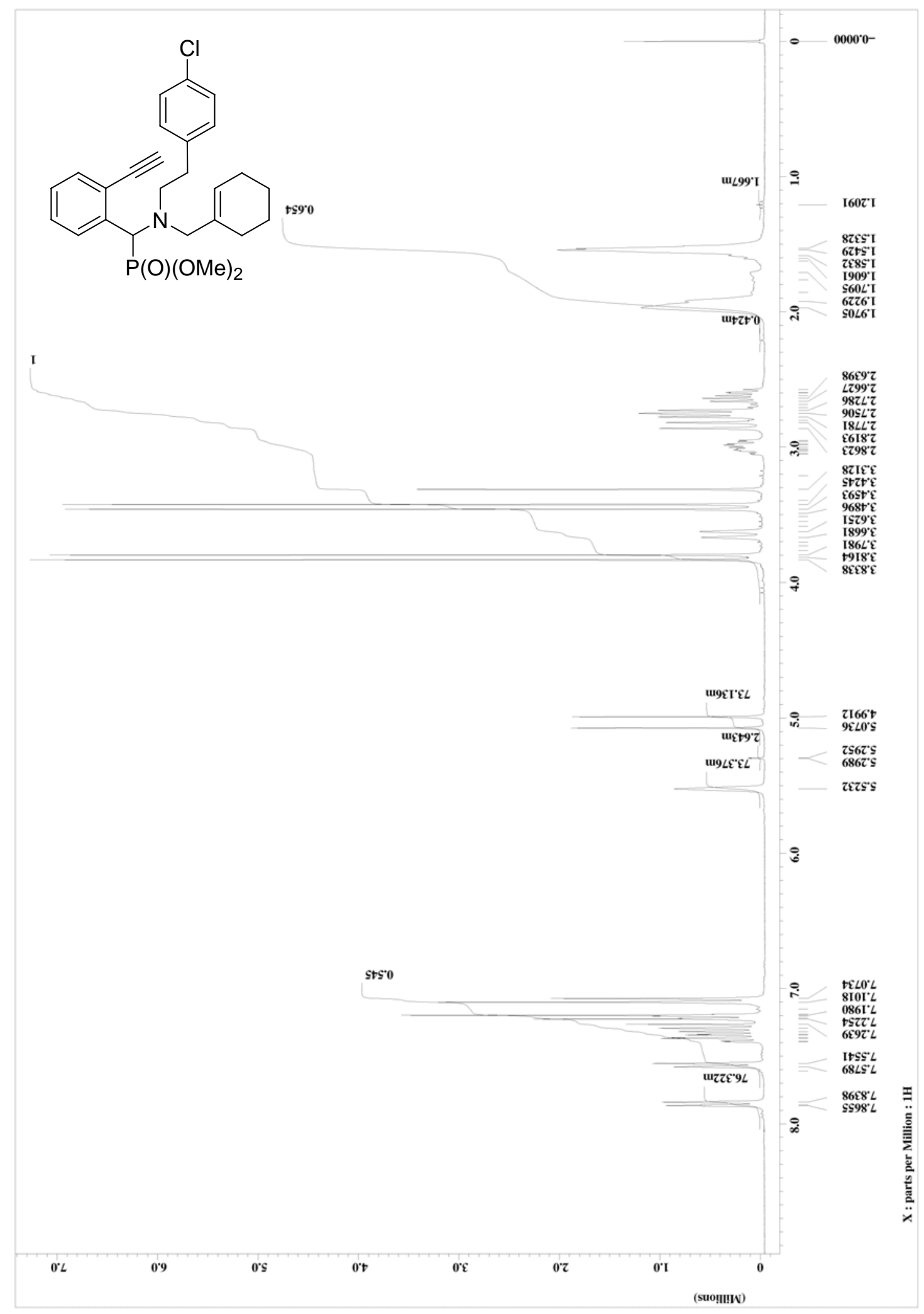


N. Dieltiens and C. V. Stevens

$\mathbf{2 h}{ }^{13} \mathrm{C}$-Spectrum

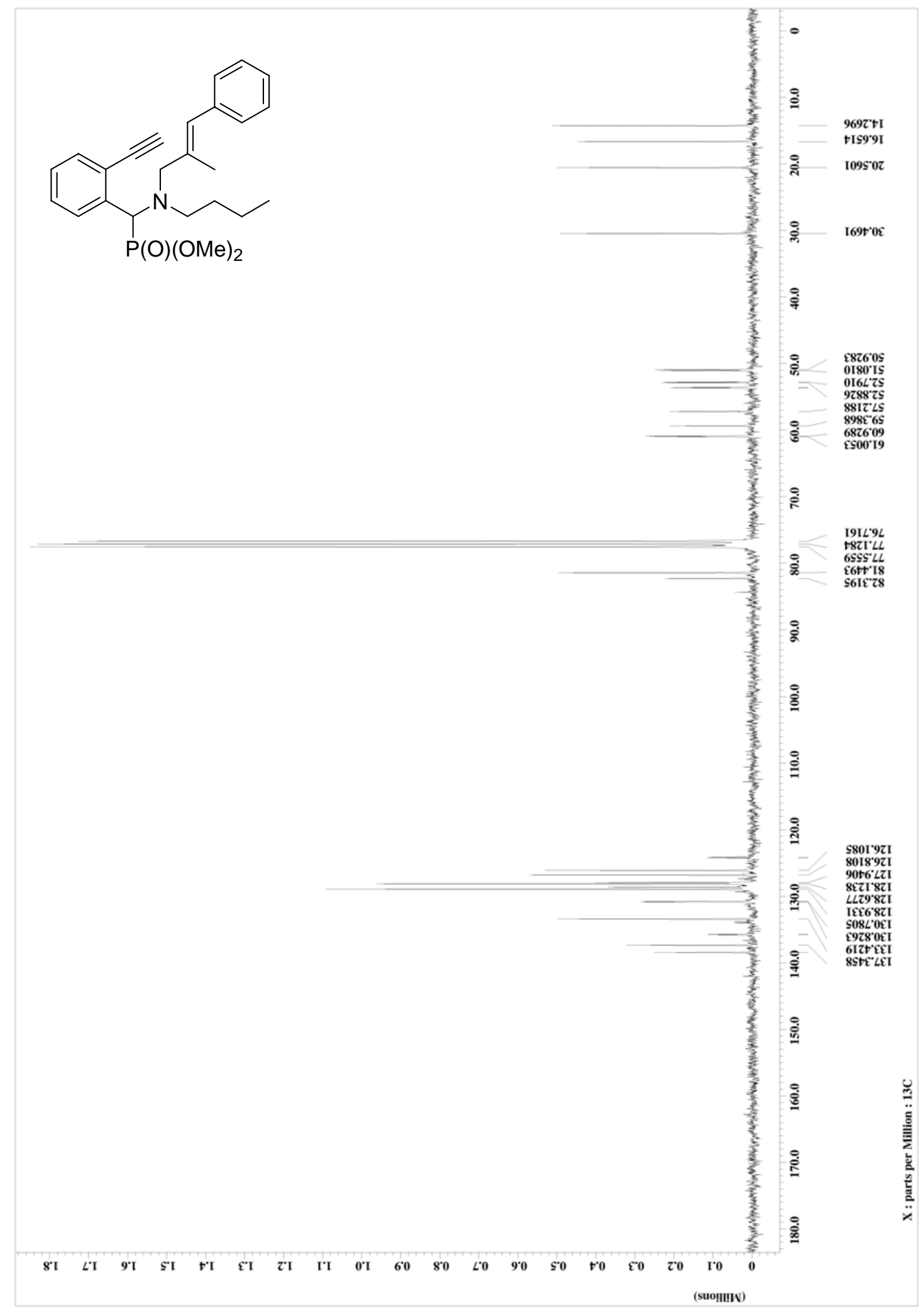


N. Dieltiens and C. V. Stevens

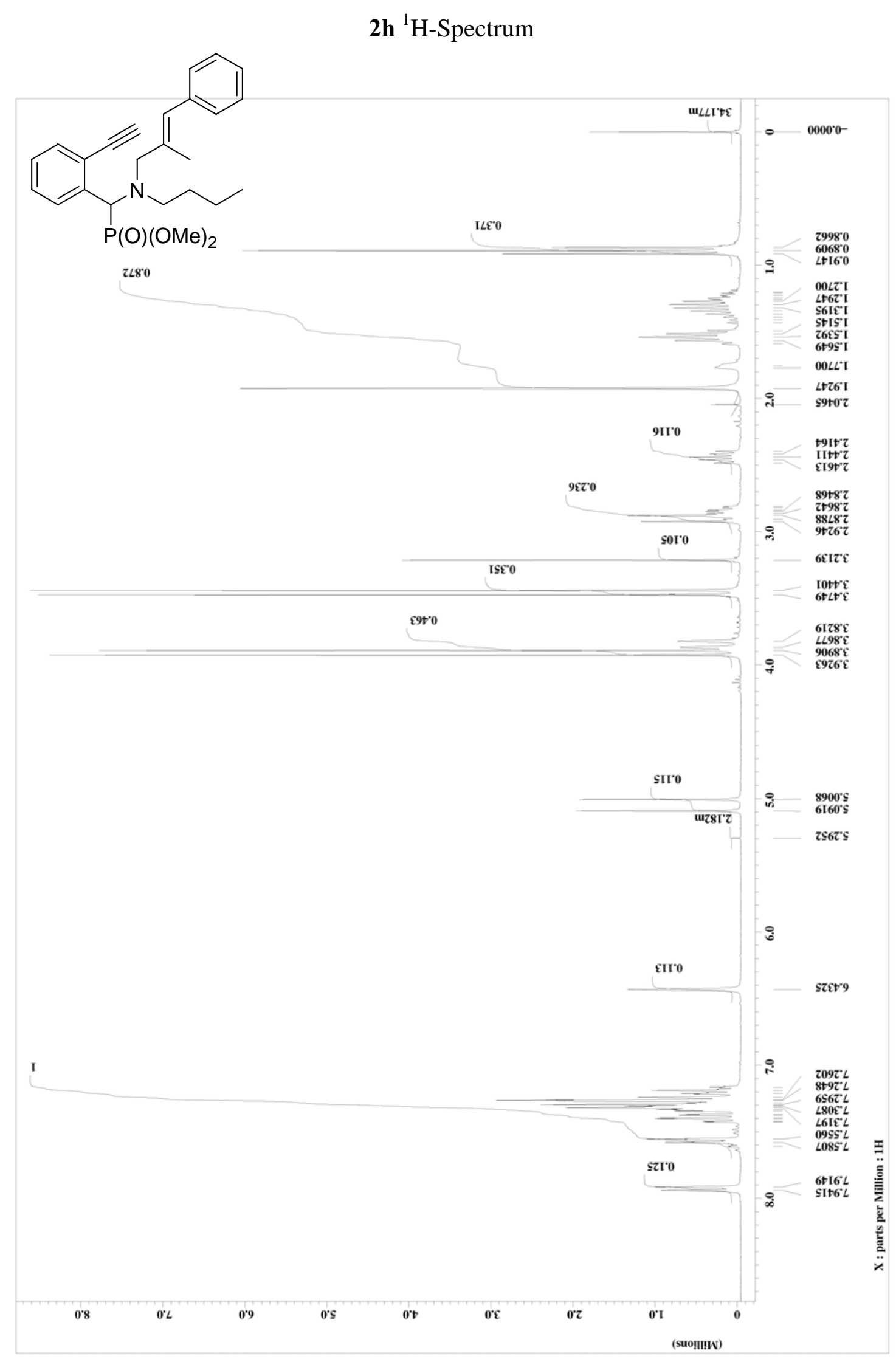


N. Dieltiens and C. V. Stevens

$2 \mathbf{i}^{13} \mathrm{C}$-Spectrum

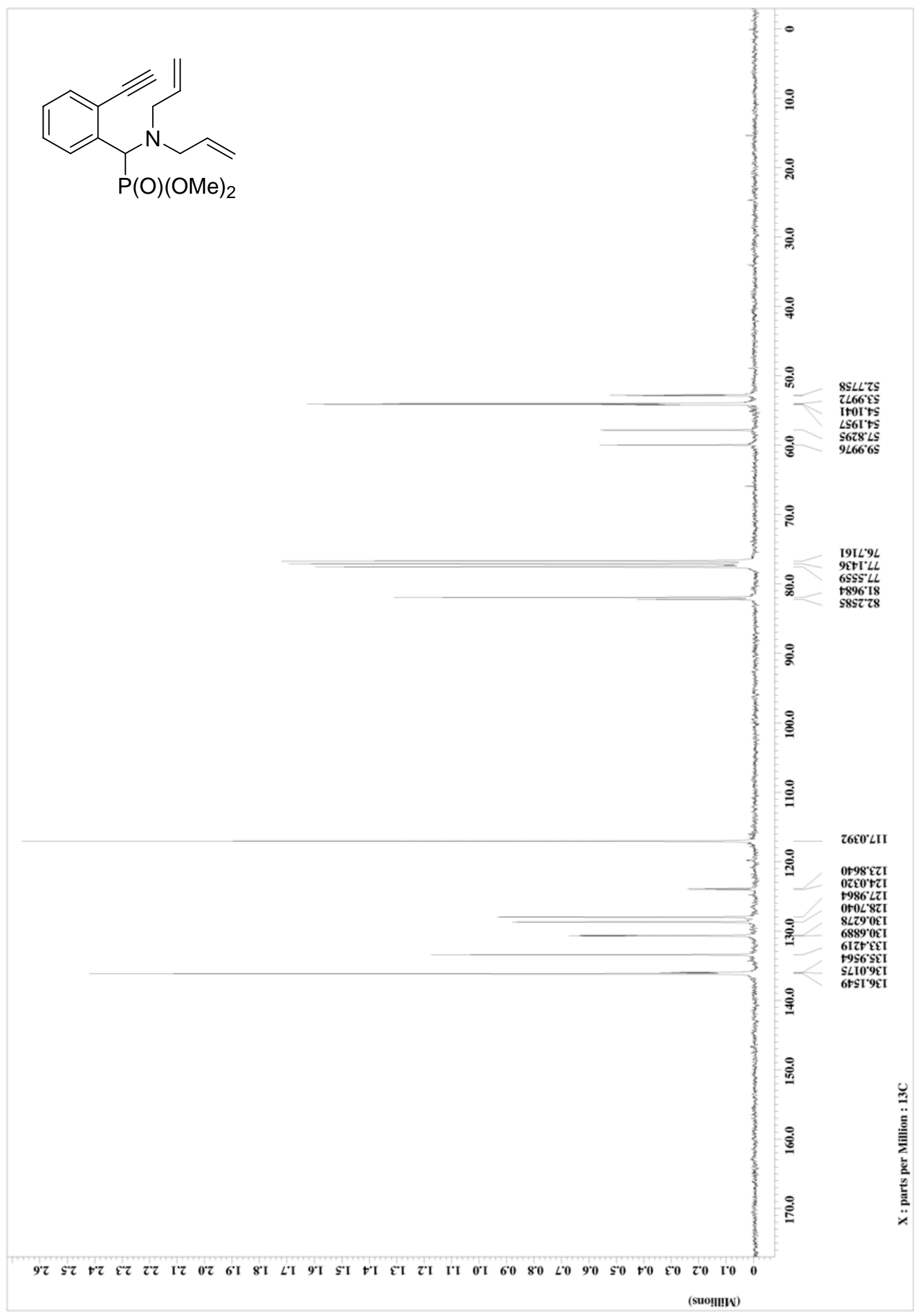

$-35-$ 
N. Dieltiens and C. V. Stevens

\section{$2 \mathbf{i}^{1} \mathrm{H}$-Spectrum}

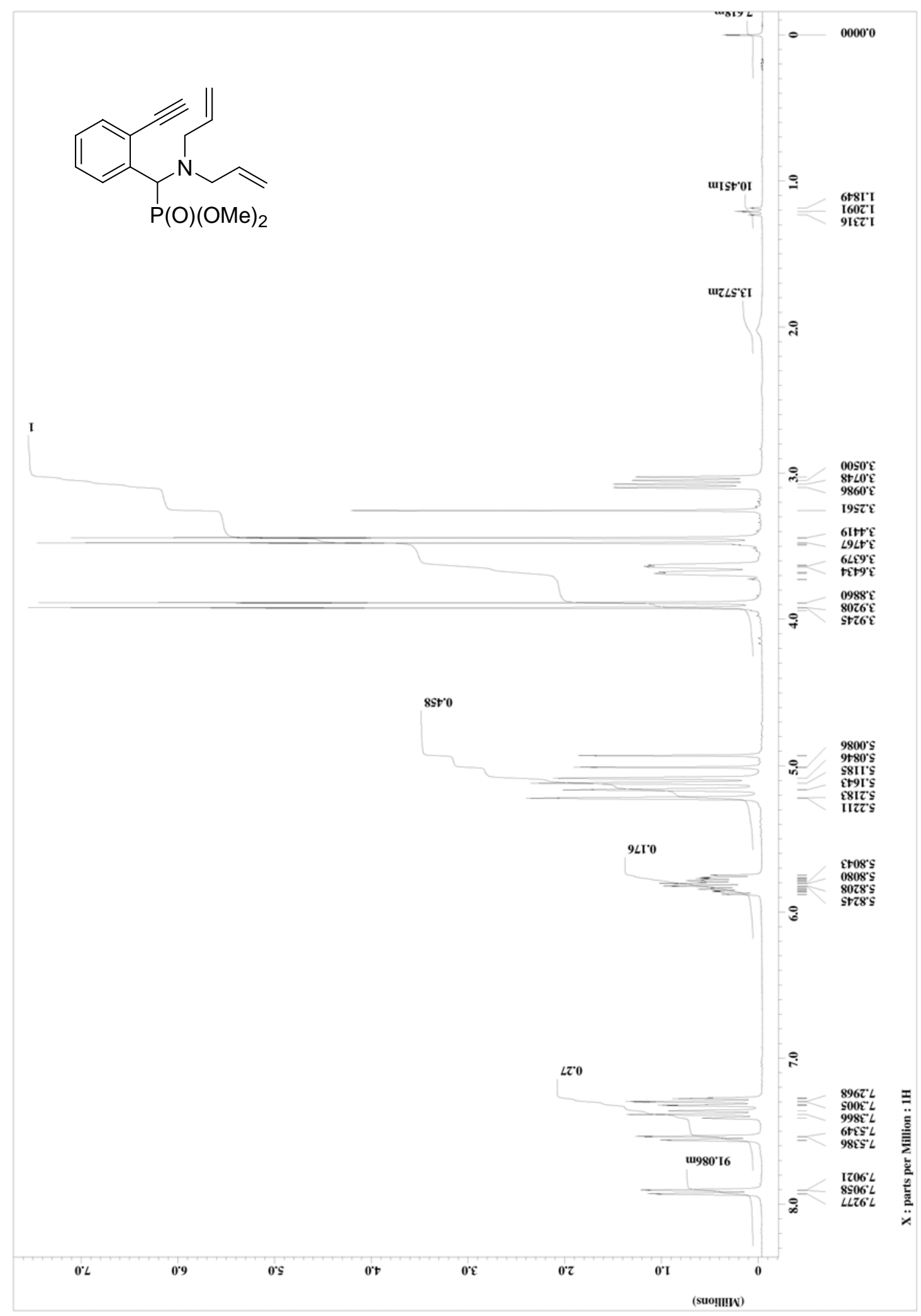


N. Dieltiens and C. V. Stevens

$\mathbf{2 j}{ }^{13} \mathrm{C}$-Spectrum

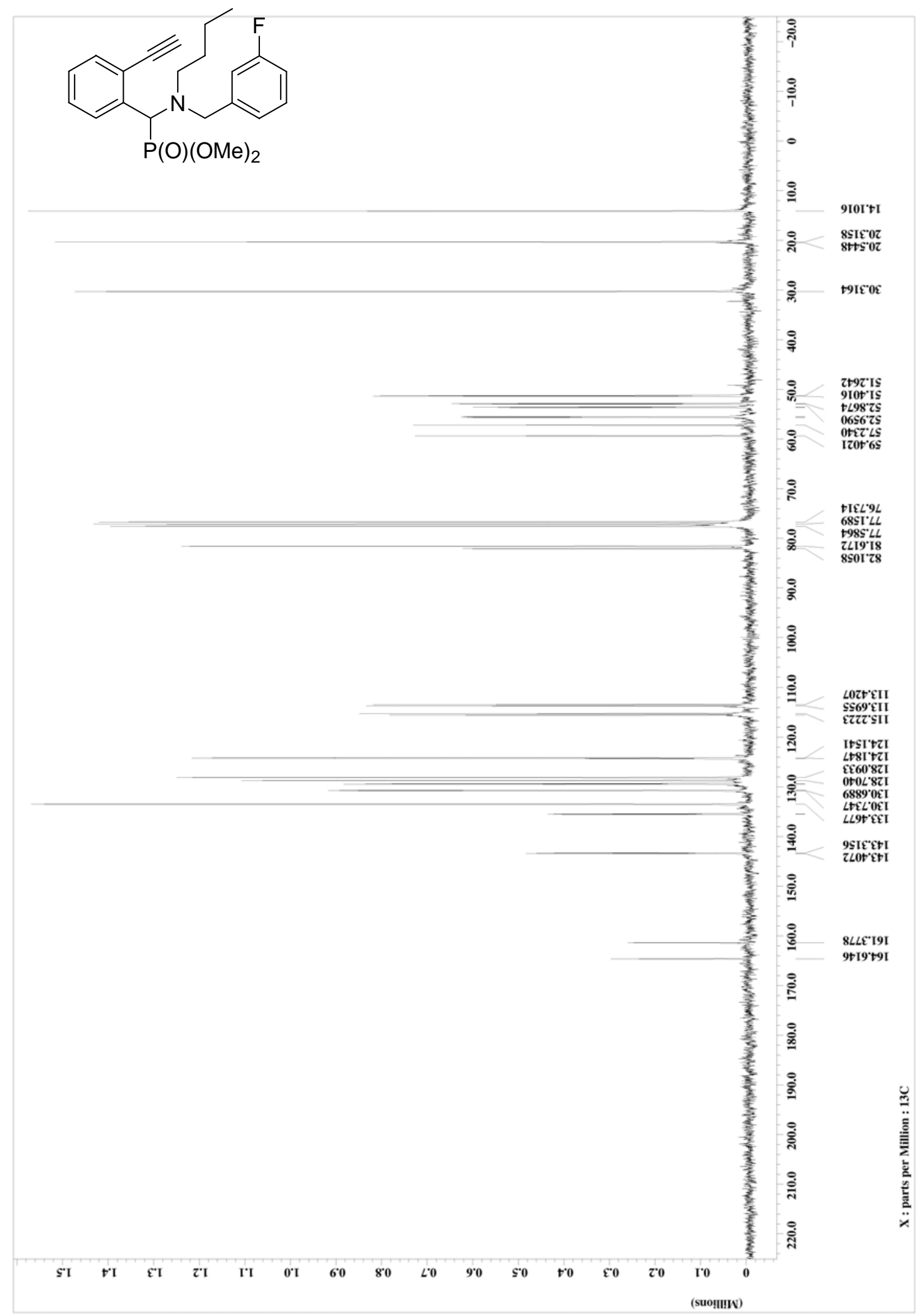


N. Dieltiens and C. V. Stevens

\section{$\mathbf{2 j}{ }^{1} \mathrm{H}$-Spectrum}

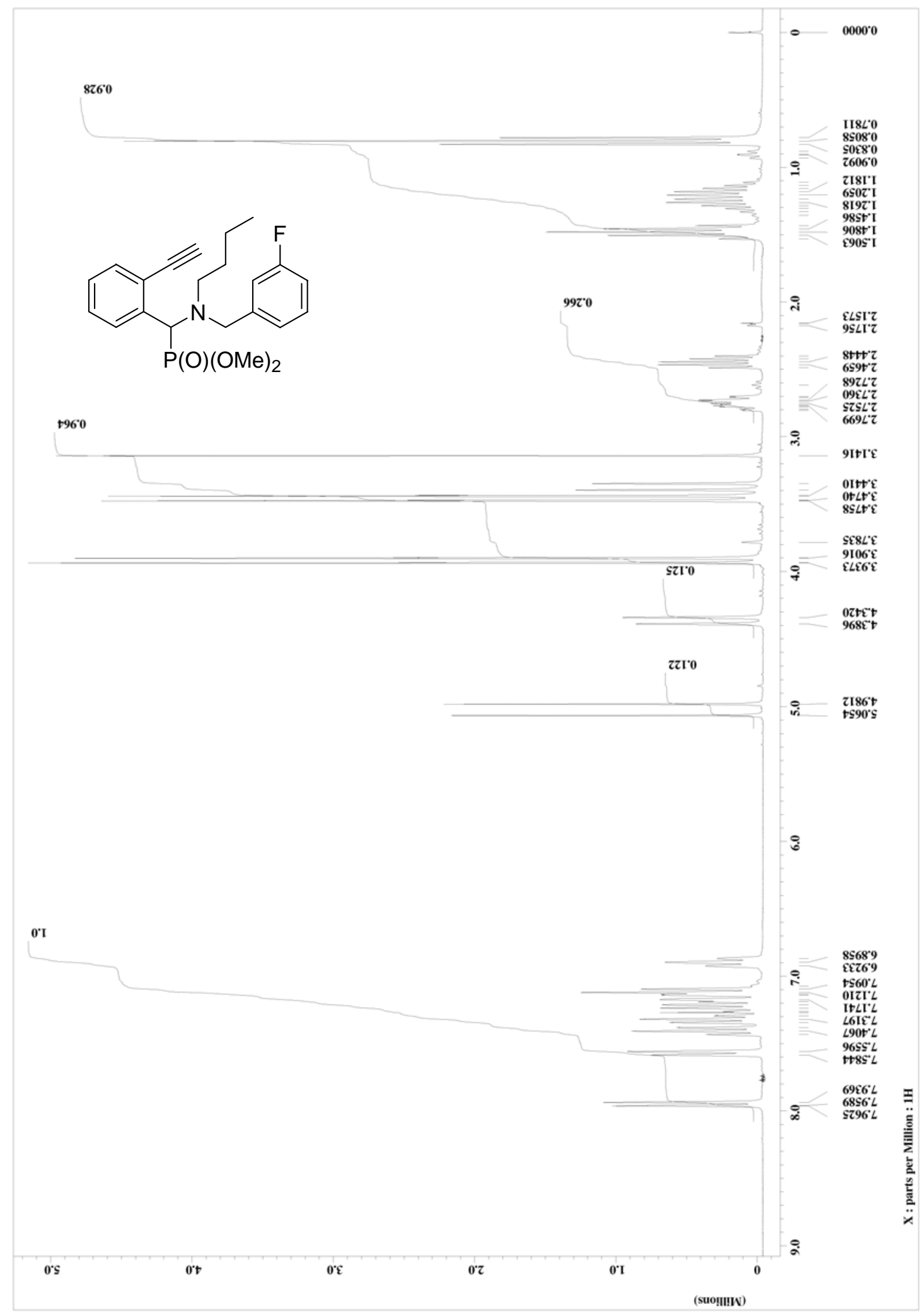


N. Dieltiens and C. V. Stevens

$\mathbf{2 k}{ }^{13} \mathrm{C}-$ Spectrum

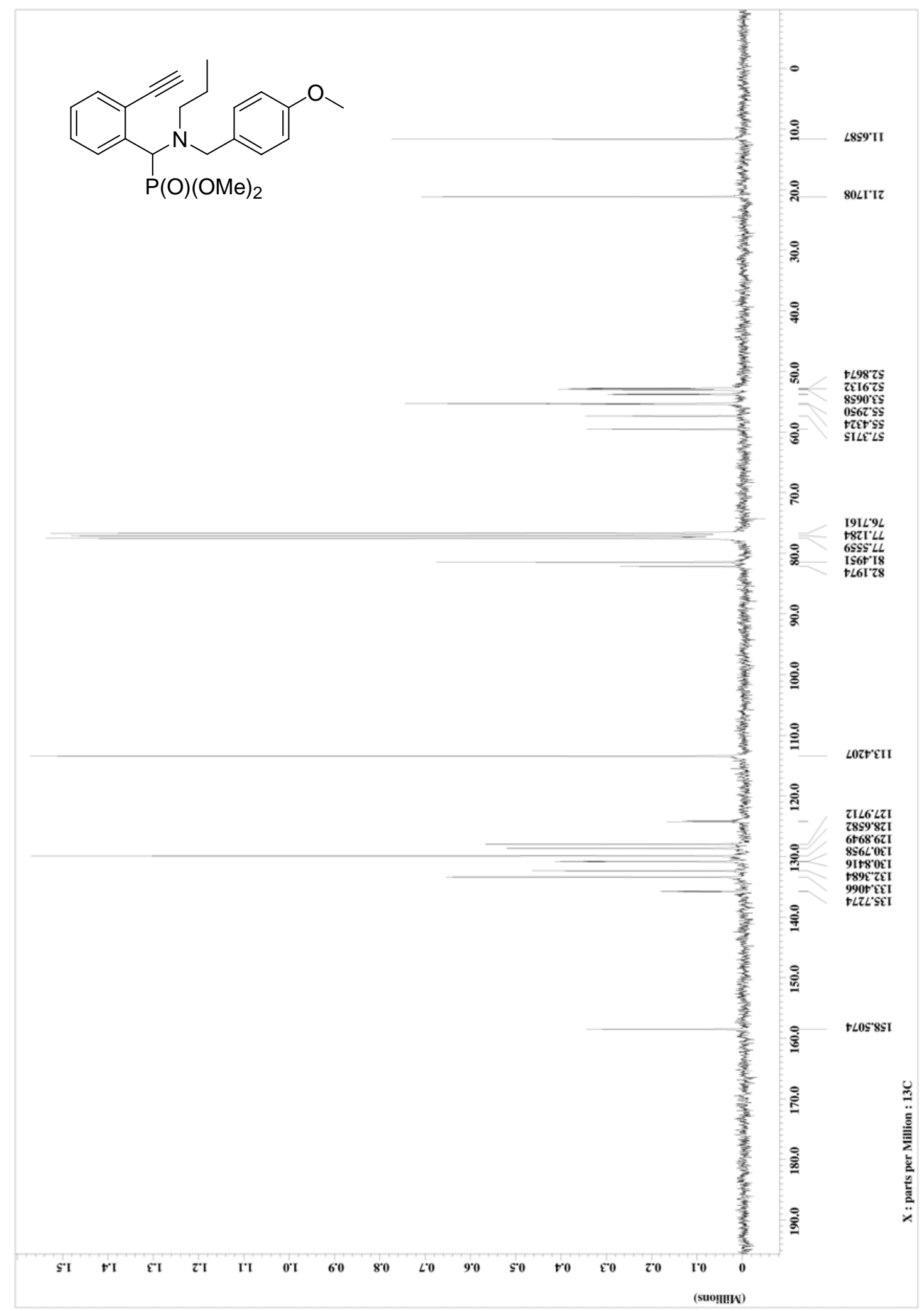


N. Dieltiens and C. V. Stevens

$\mathbf{2 k}{ }^{1} \mathrm{H}$-Spectrum

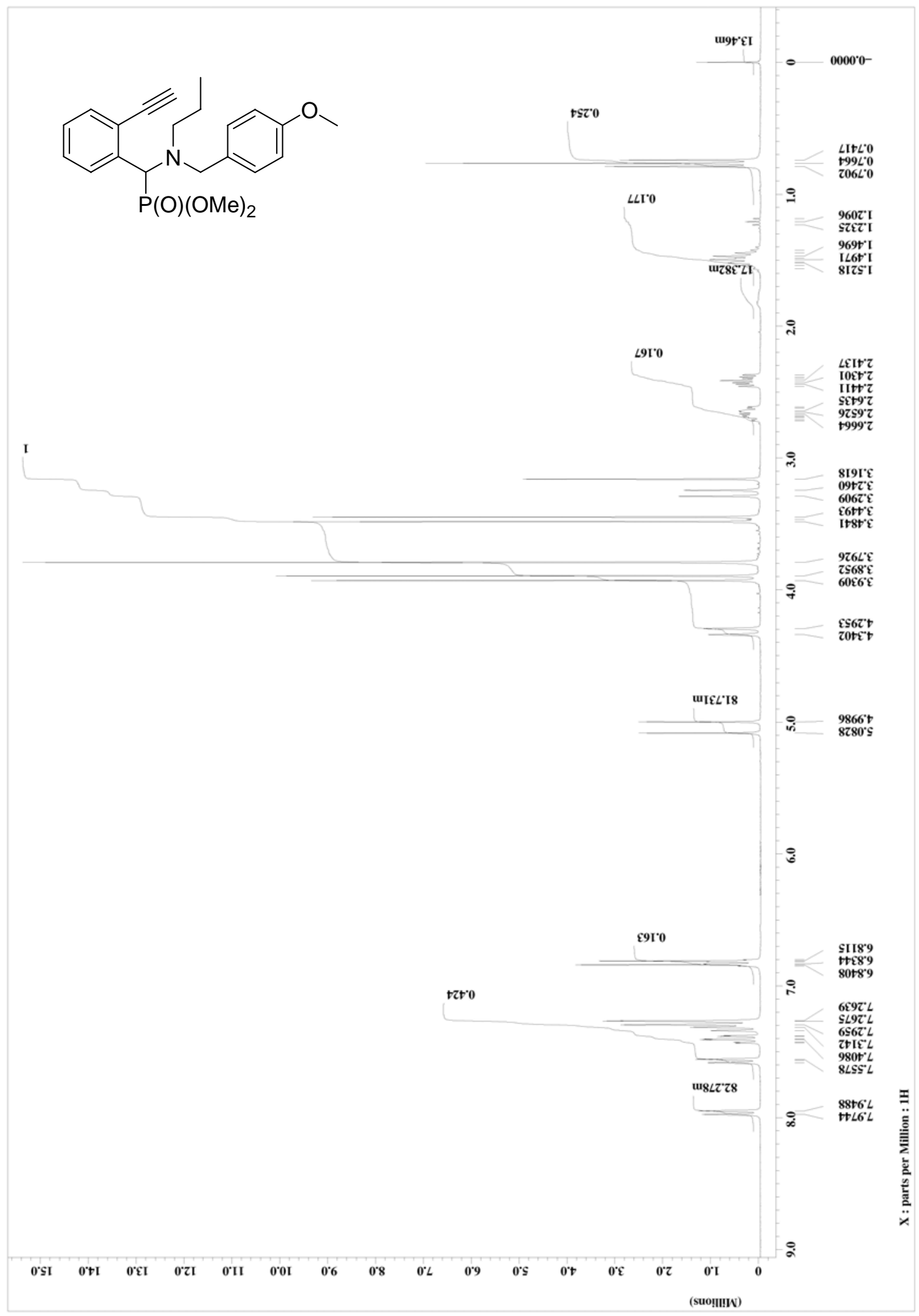

$-40-$ 
N. Dieltiens and C. V. Stevens

$2 \mathbf{I}^{13} \mathrm{C}$-Spectrum

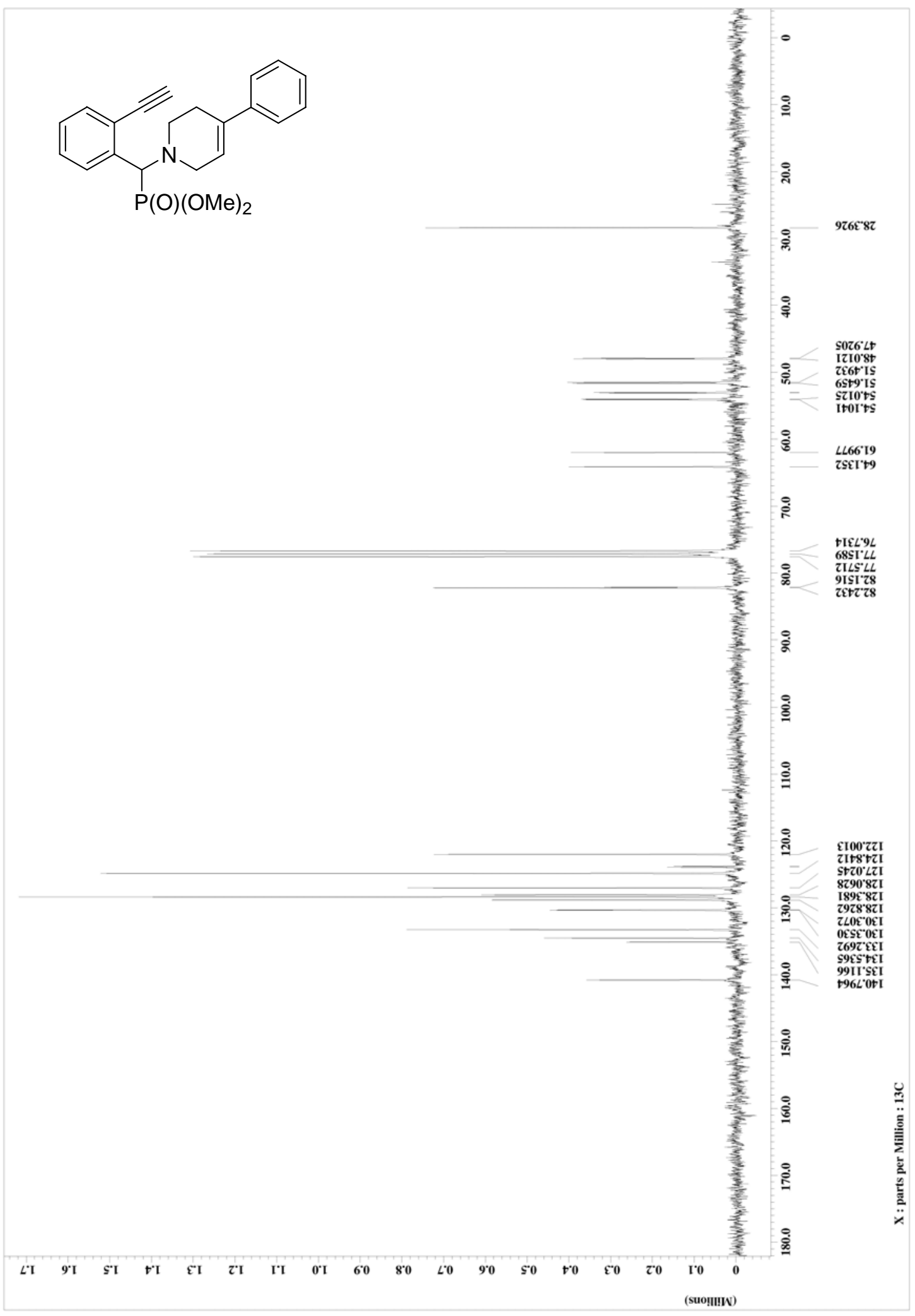


N. Dieltiens and C. V. Stevens

2l ${ }^{1} \mathrm{H}-S p e c t r u m$

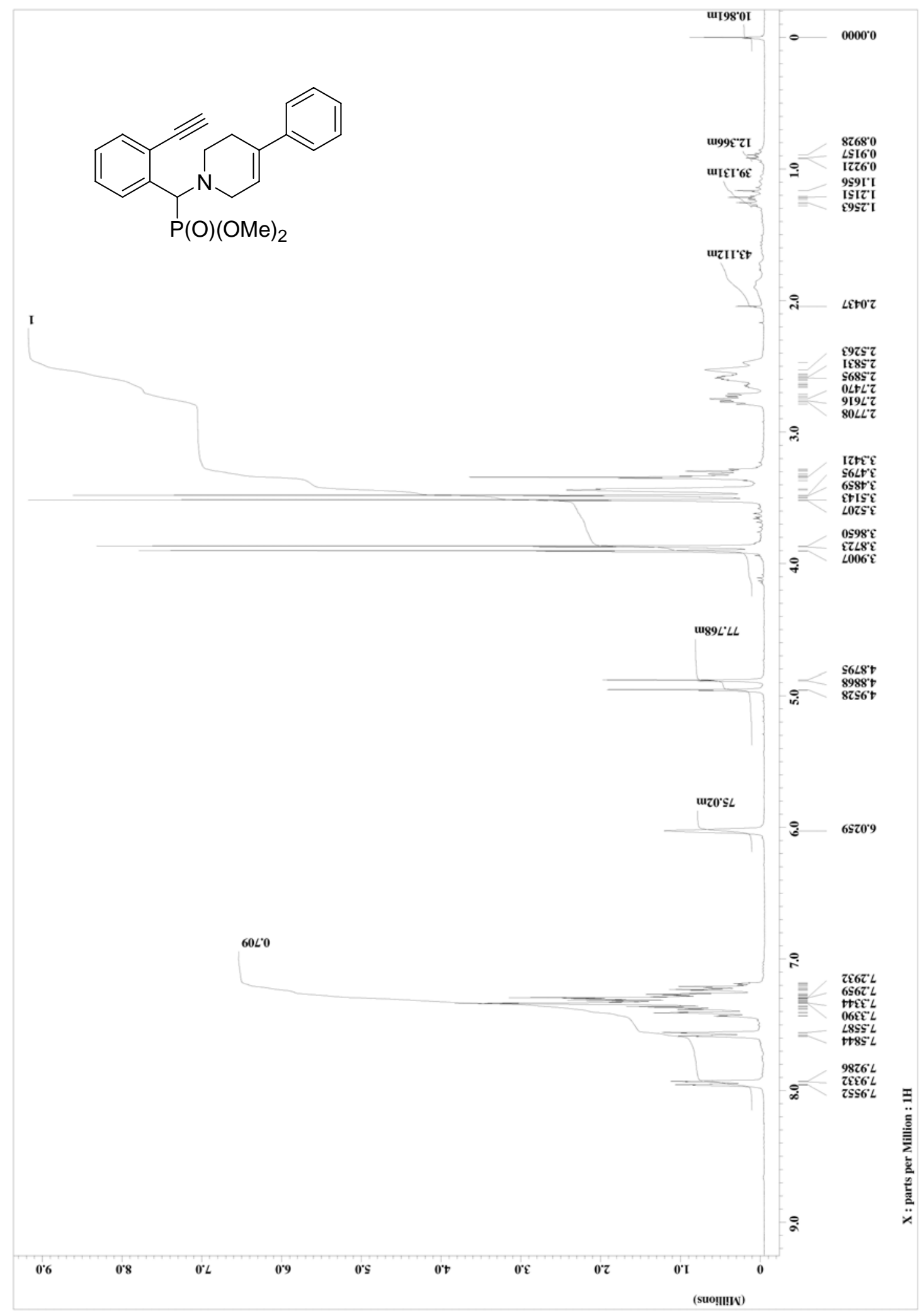


N. Dieltiens and C. V. Stevens

\section{$\mathbf{2 m}{ }^{13}$ C-Spectrum}

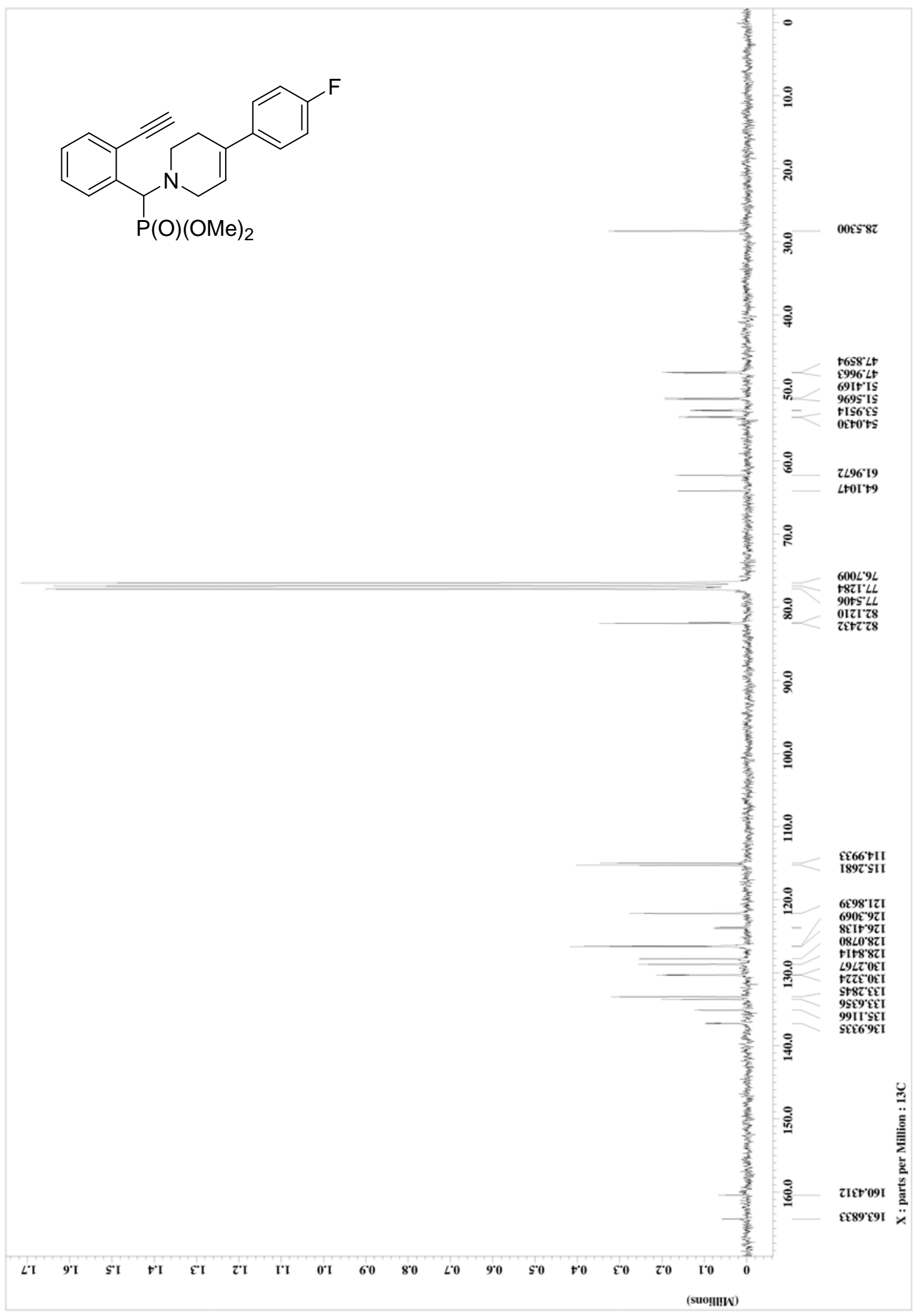


N. Dieltiens and C. V. Stevens

$\mathbf{2 m}{ }^{1} \mathrm{H}$-Spectrum

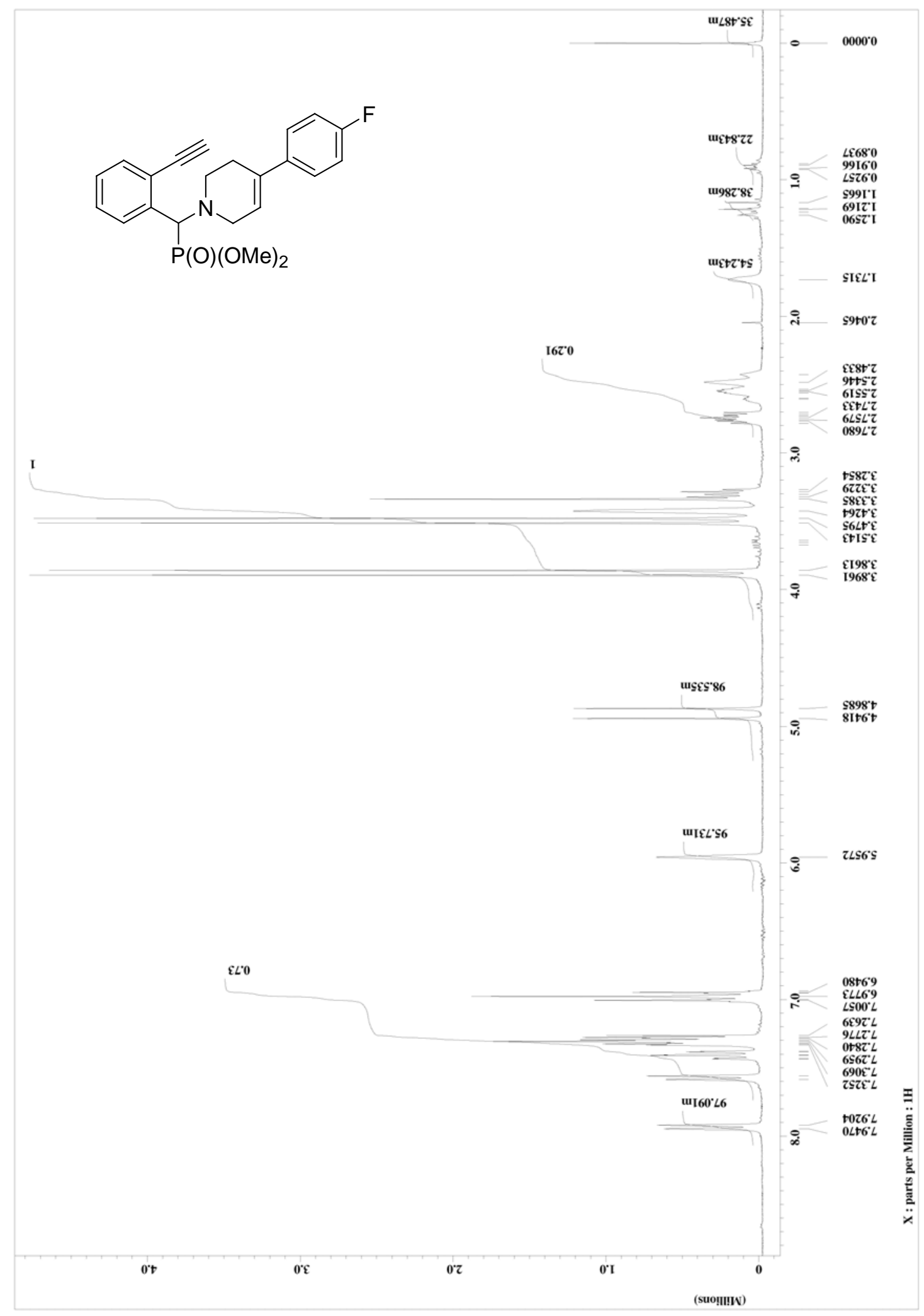


N. Dieltiens and C. V. Stevens

4a ${ }^{13} \mathrm{C}-$ Spectrum

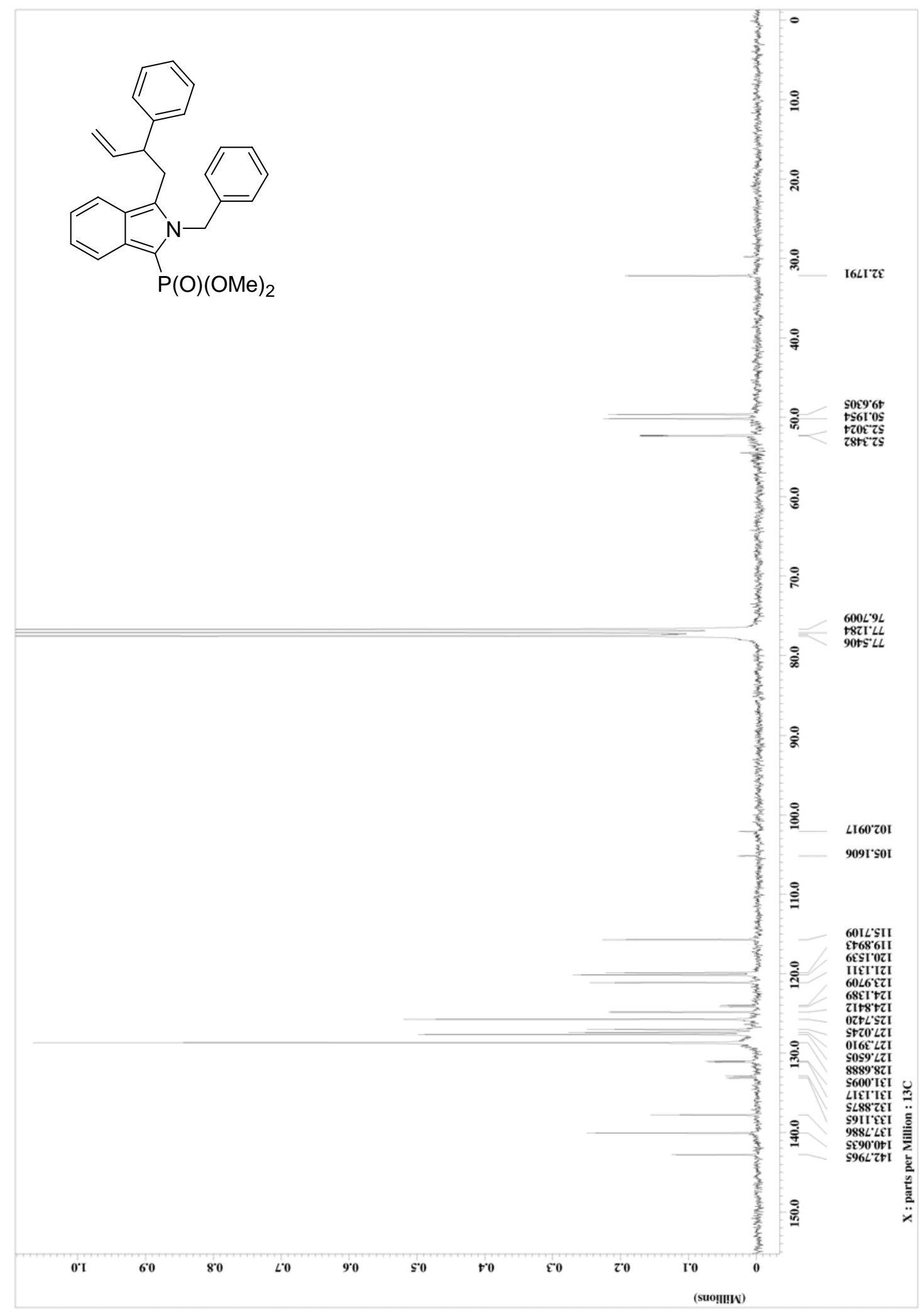


N. Dieltiens and C. V. Stevens

4a ${ }^{1} \mathrm{H}$-Spectrum

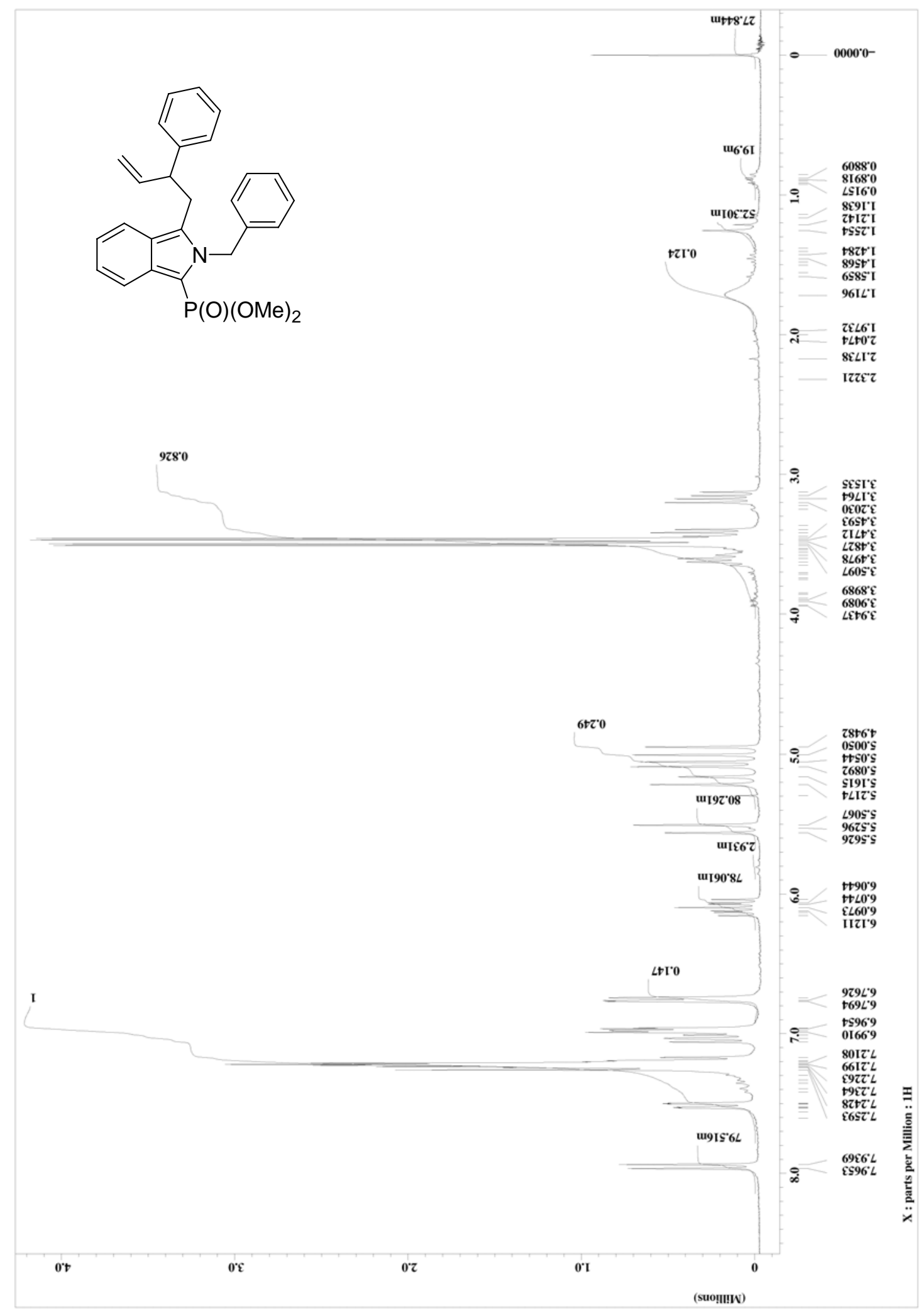


N. Dieltiens and C. V. Stevens

\section{4b ${ }^{13} \mathrm{C}$-Spectrum}

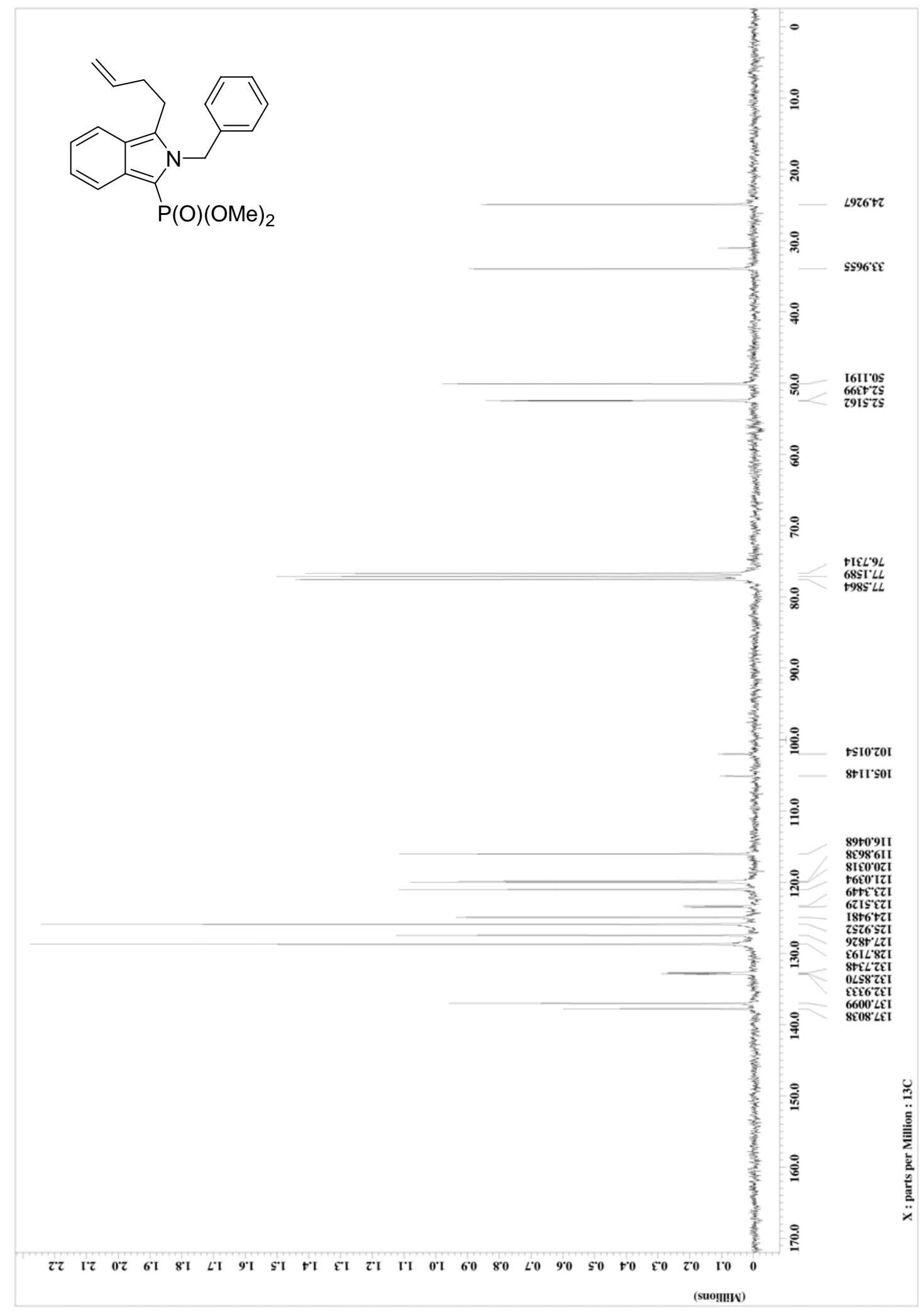


N. Dieltiens and C. V. Stevens

4b ${ }^{1} \mathrm{H}$-Spectrum

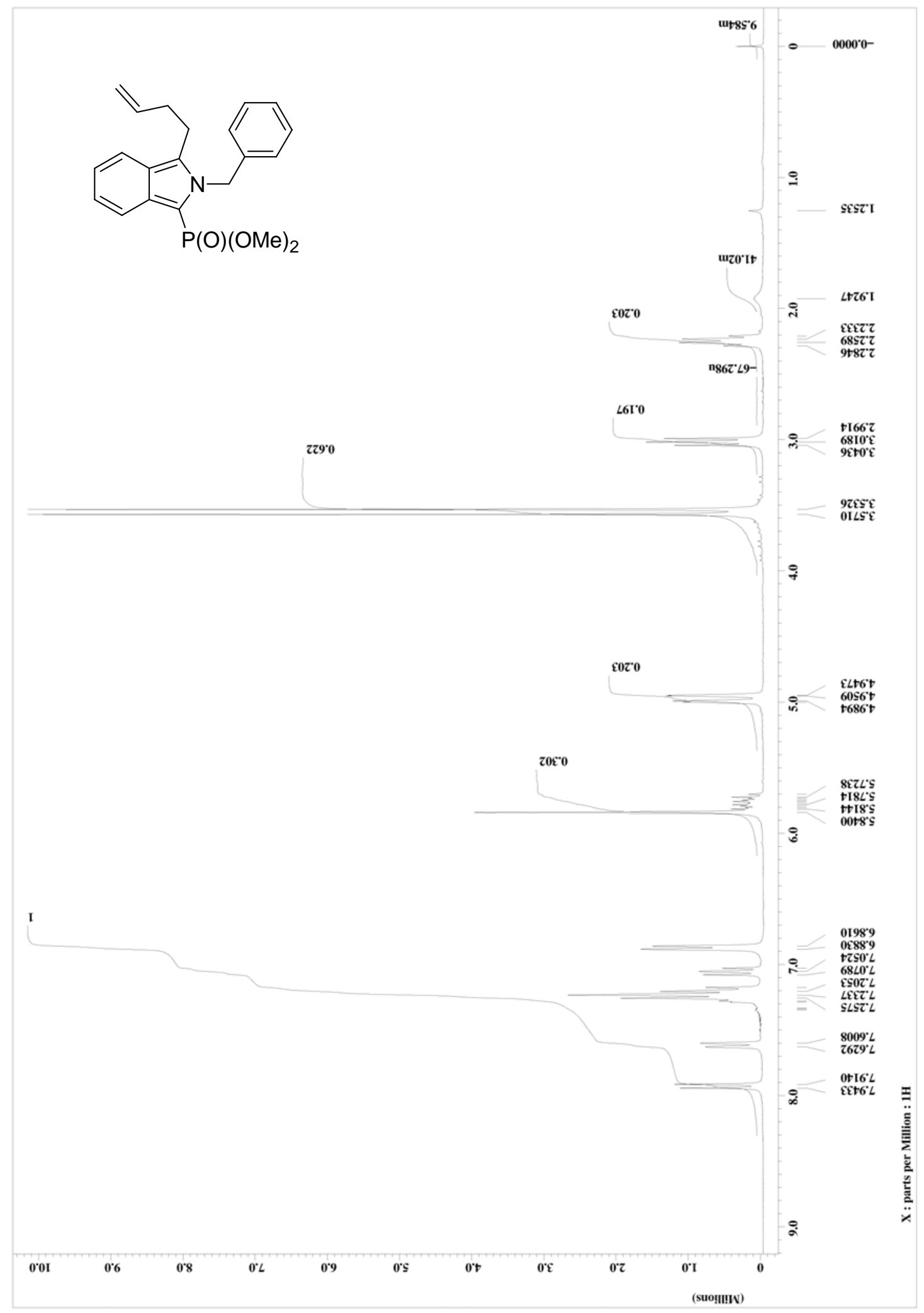


N. Dieltiens and C. V. Stevens

4c ${ }^{13} \mathrm{C}$-Spectrum

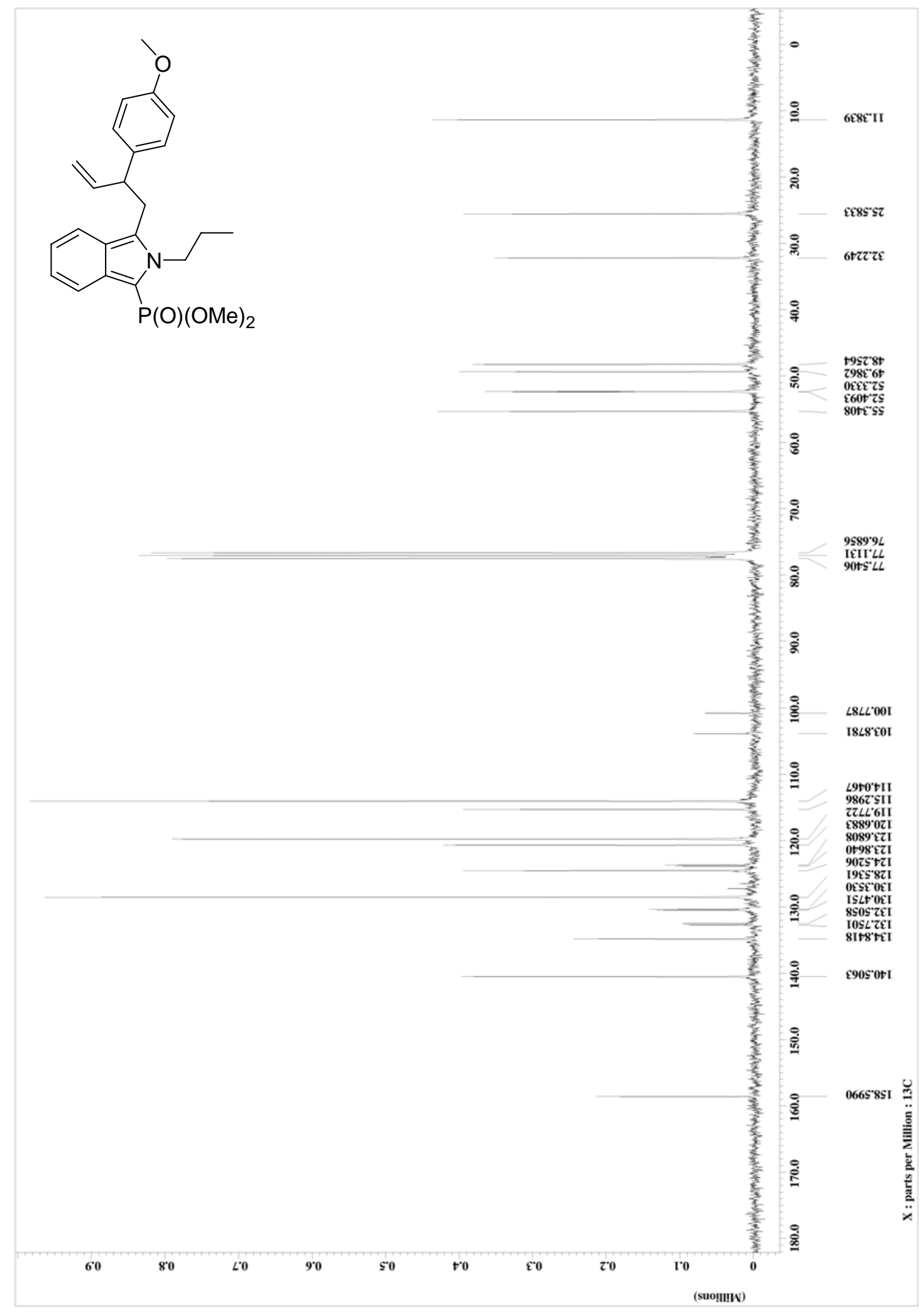


N. Dieltiens and C. V. Stevens

4c ${ }^{1} \mathrm{H}$-Spectrum

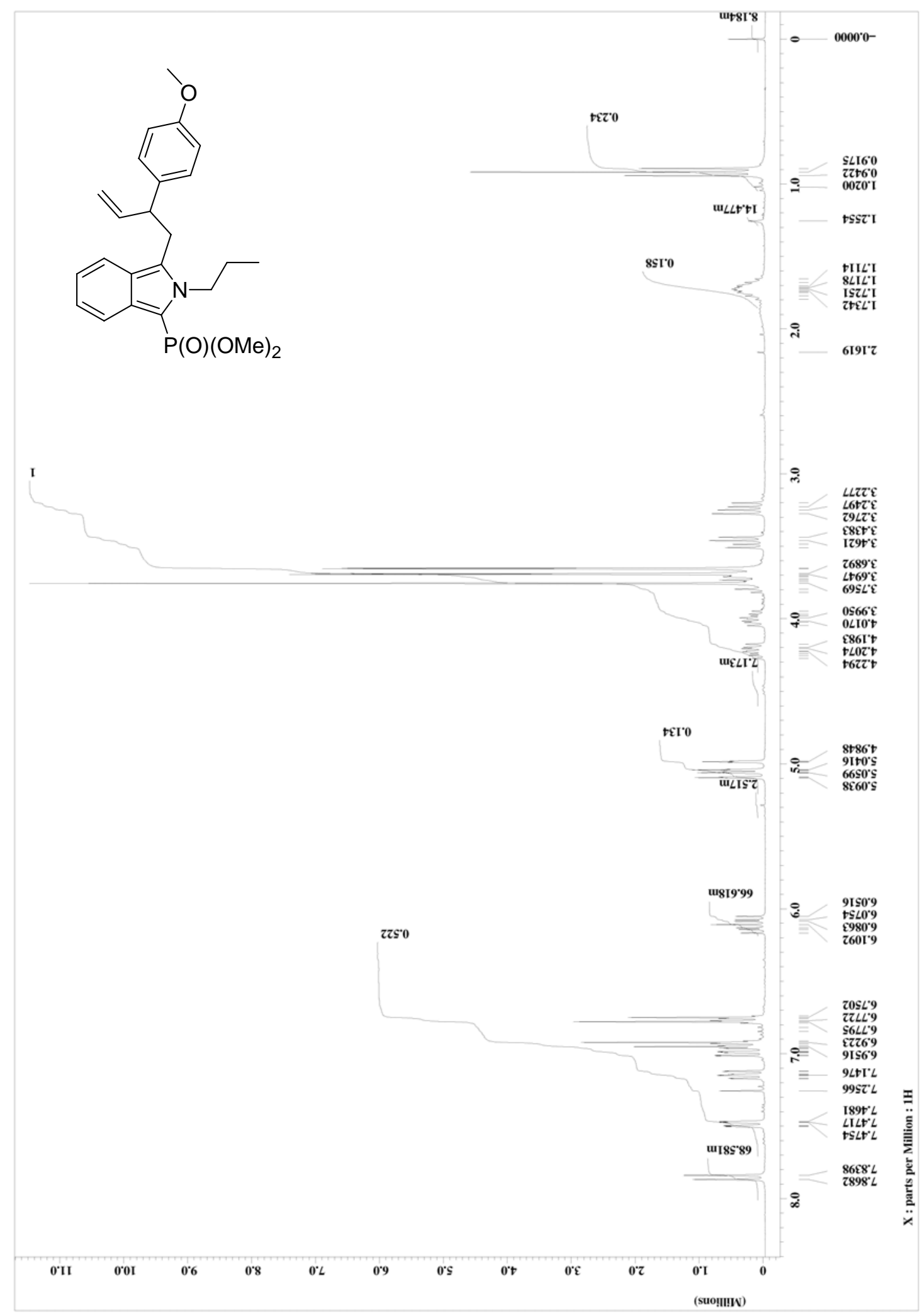


N. Dieltiens and C. V. Stevens

4d ${ }^{13} \mathrm{C}-$ Spectrum

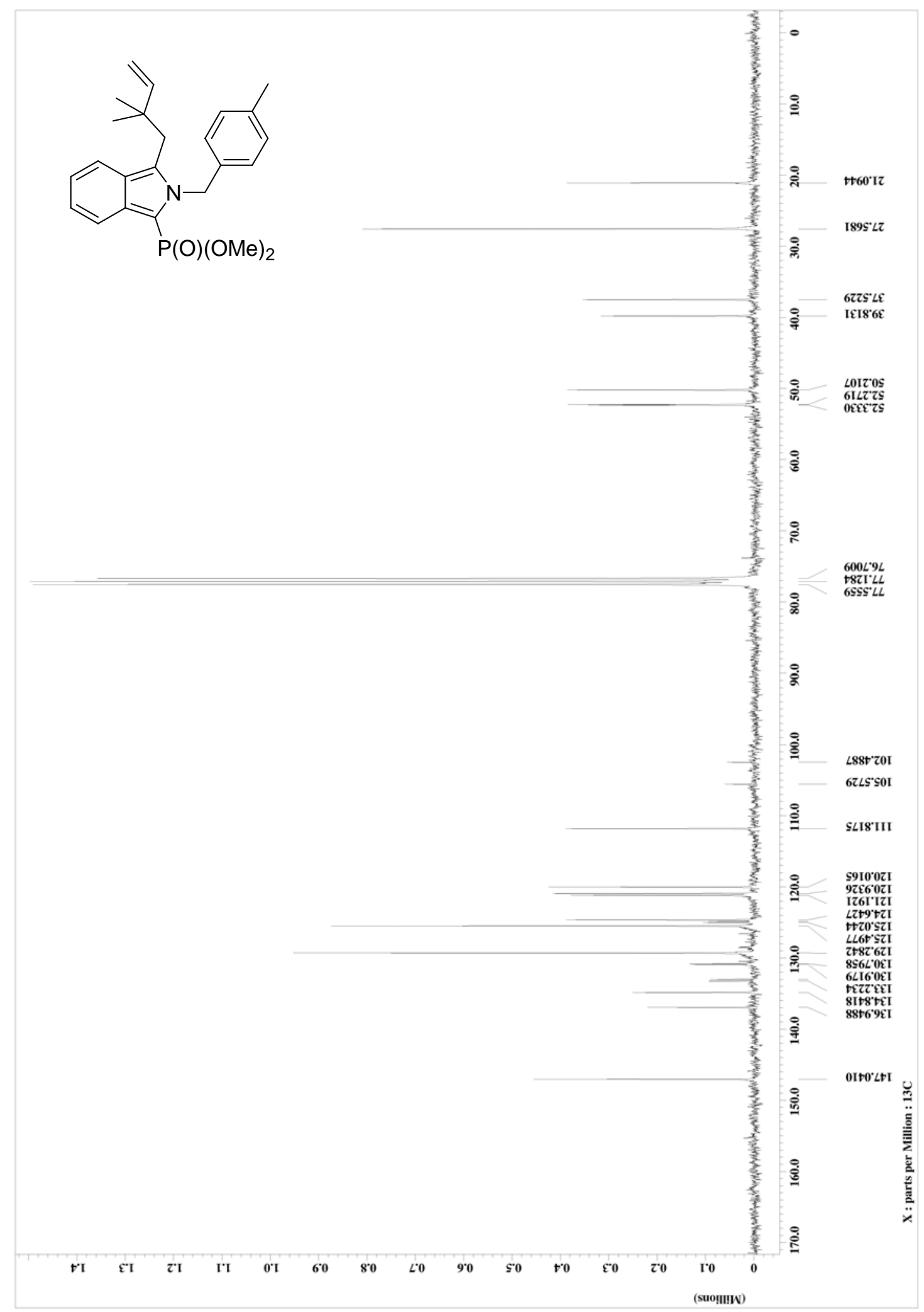


N. Dieltiens and C. V. Stevens

4d ${ }^{1} \mathrm{H}$-Spectrum

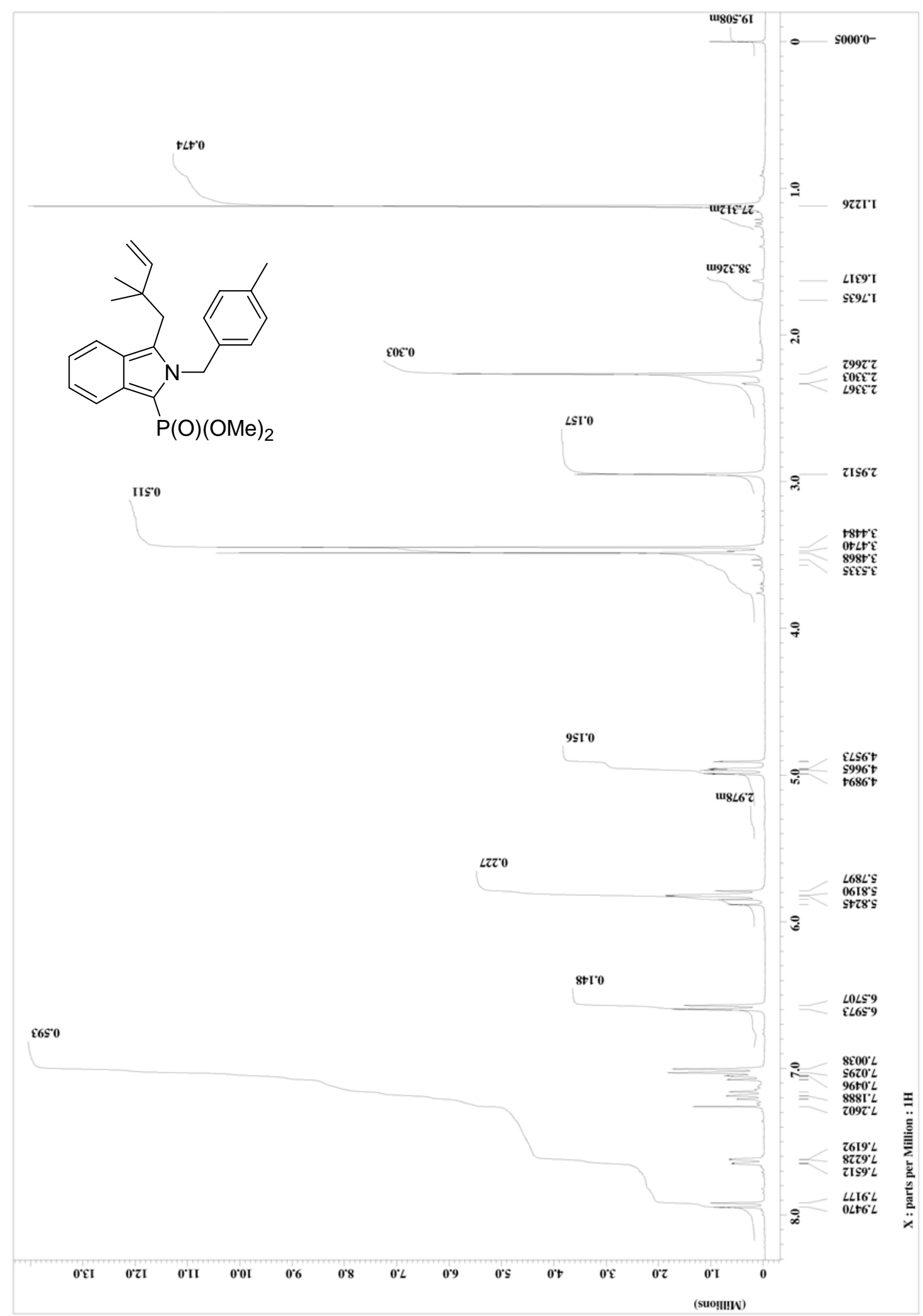


N. Dieltiens and C. V. Stevens

4e ${ }^{13} \mathrm{C}$-Spectrum

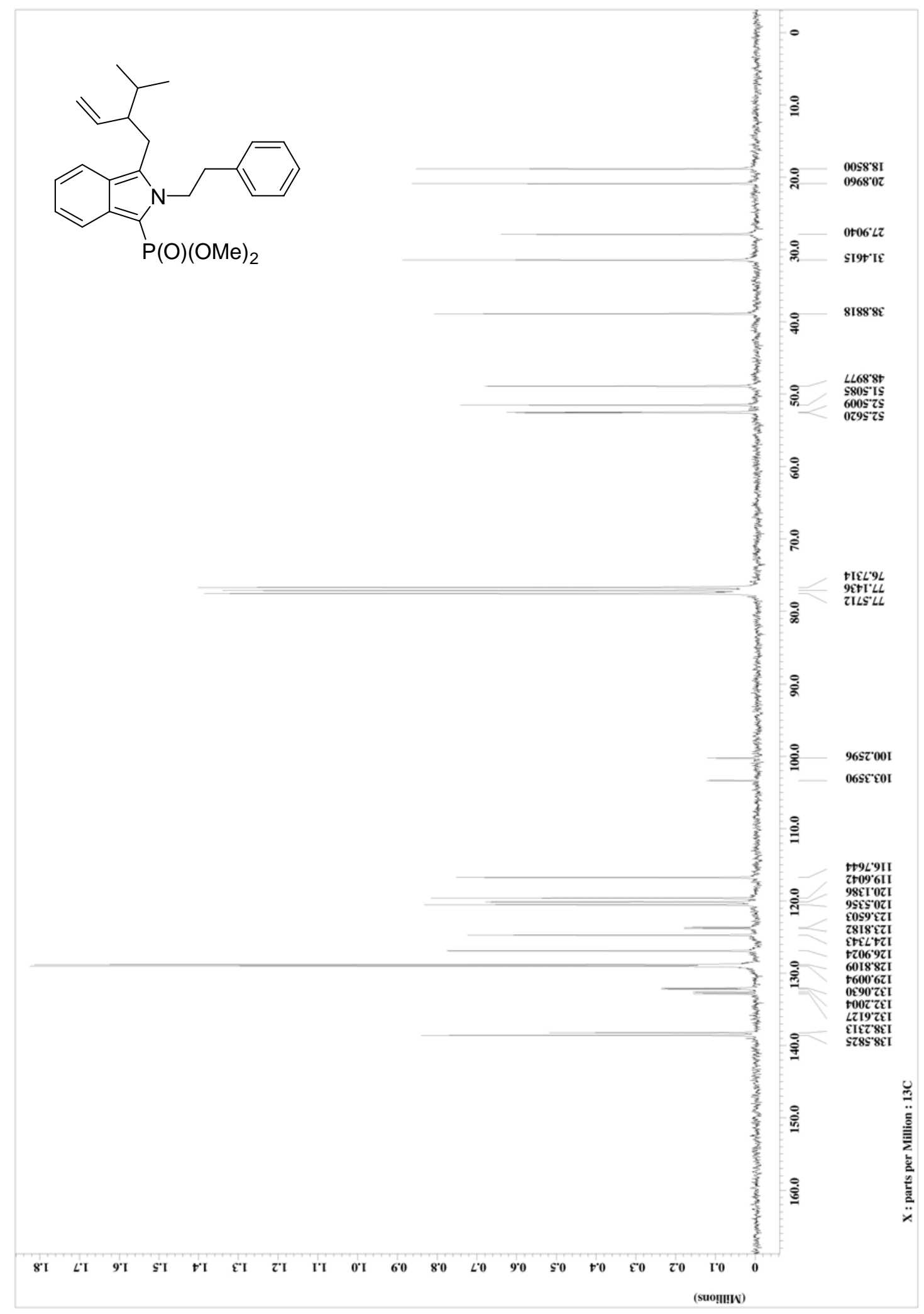


N. Dieltiens and C. V. Stevens

4e ${ }^{1} \mathrm{H}$-Spectrum

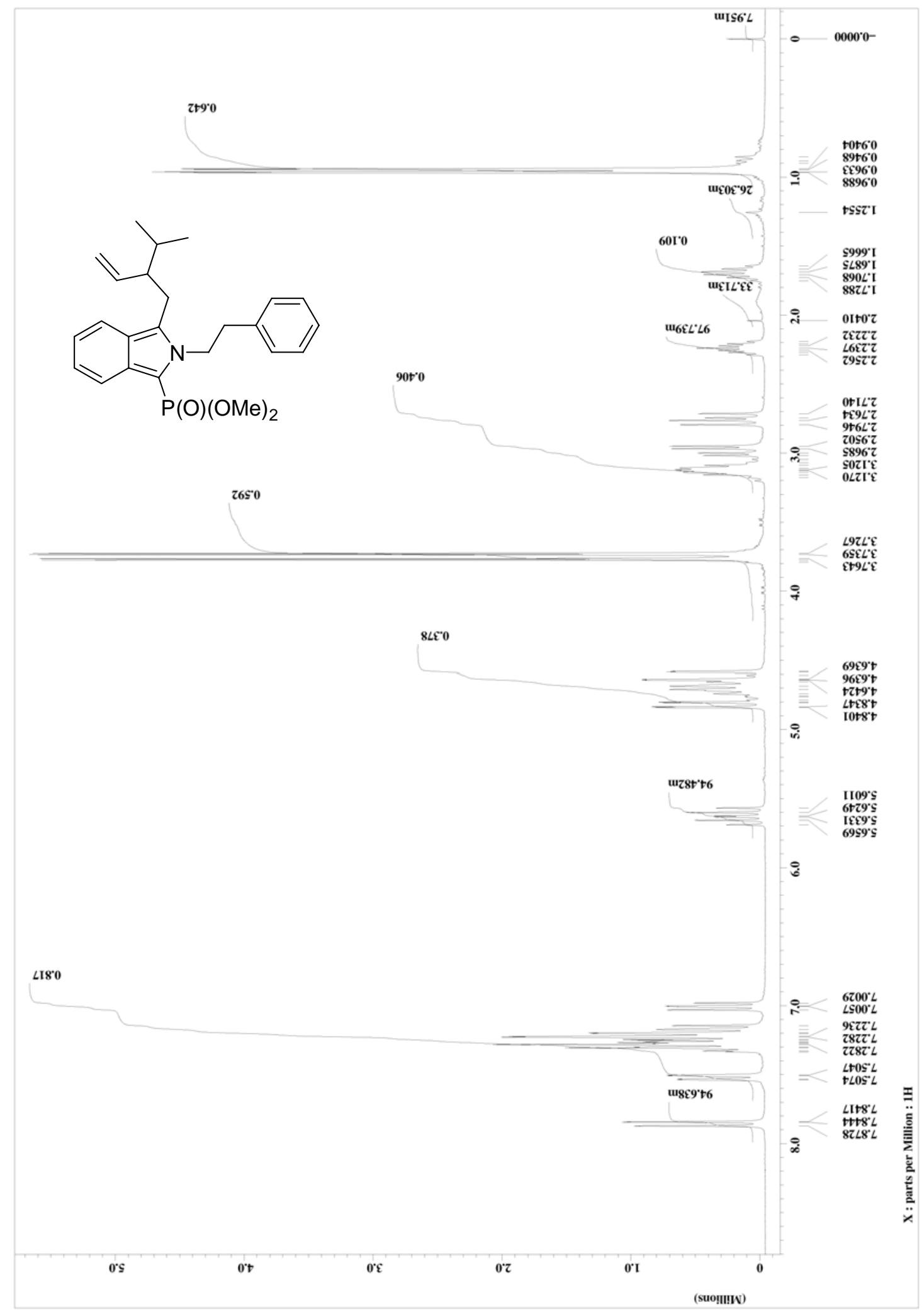


N. Dieltiens and C. V. Stevens

$$
4 f^{13} \mathrm{C} \text {-Spectrum }
$$

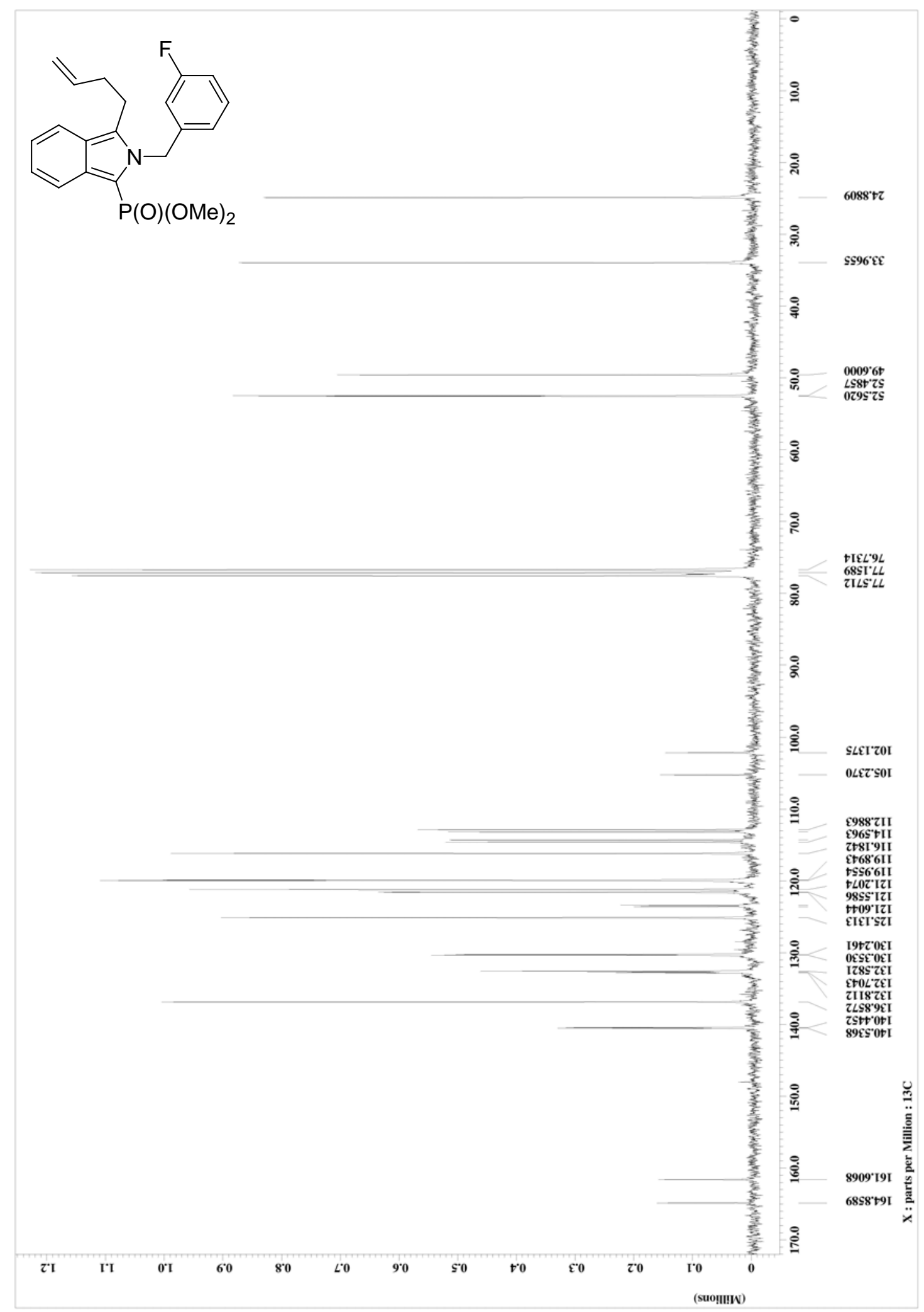


N. Dieltiens and C. V. Stevens

\section{4f ${ }^{1} \mathrm{H}$-Spectrum}

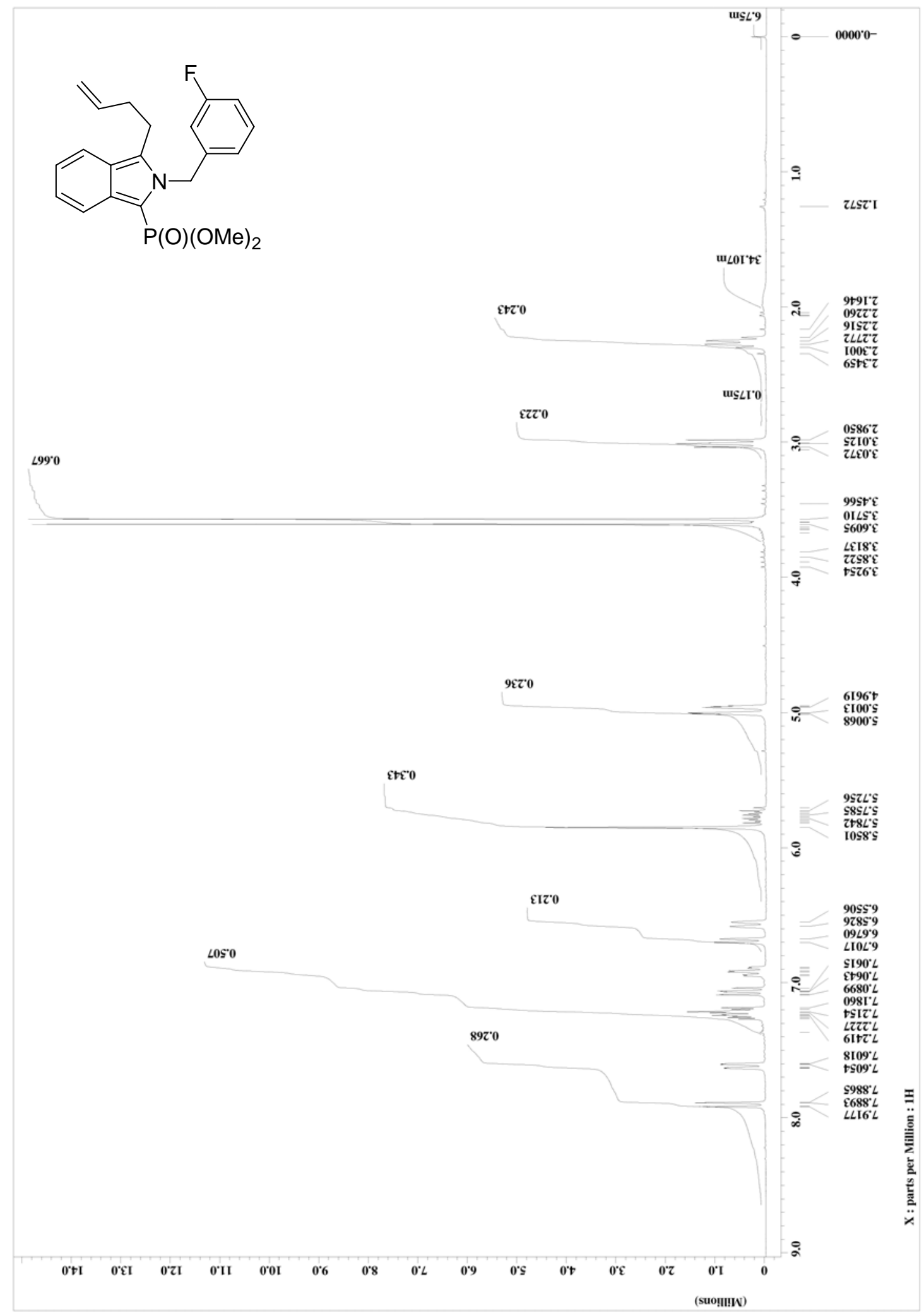


N. Dieltiens and C. V. Stevens

4g ${ }^{13} \mathrm{C}-$ Spectrum

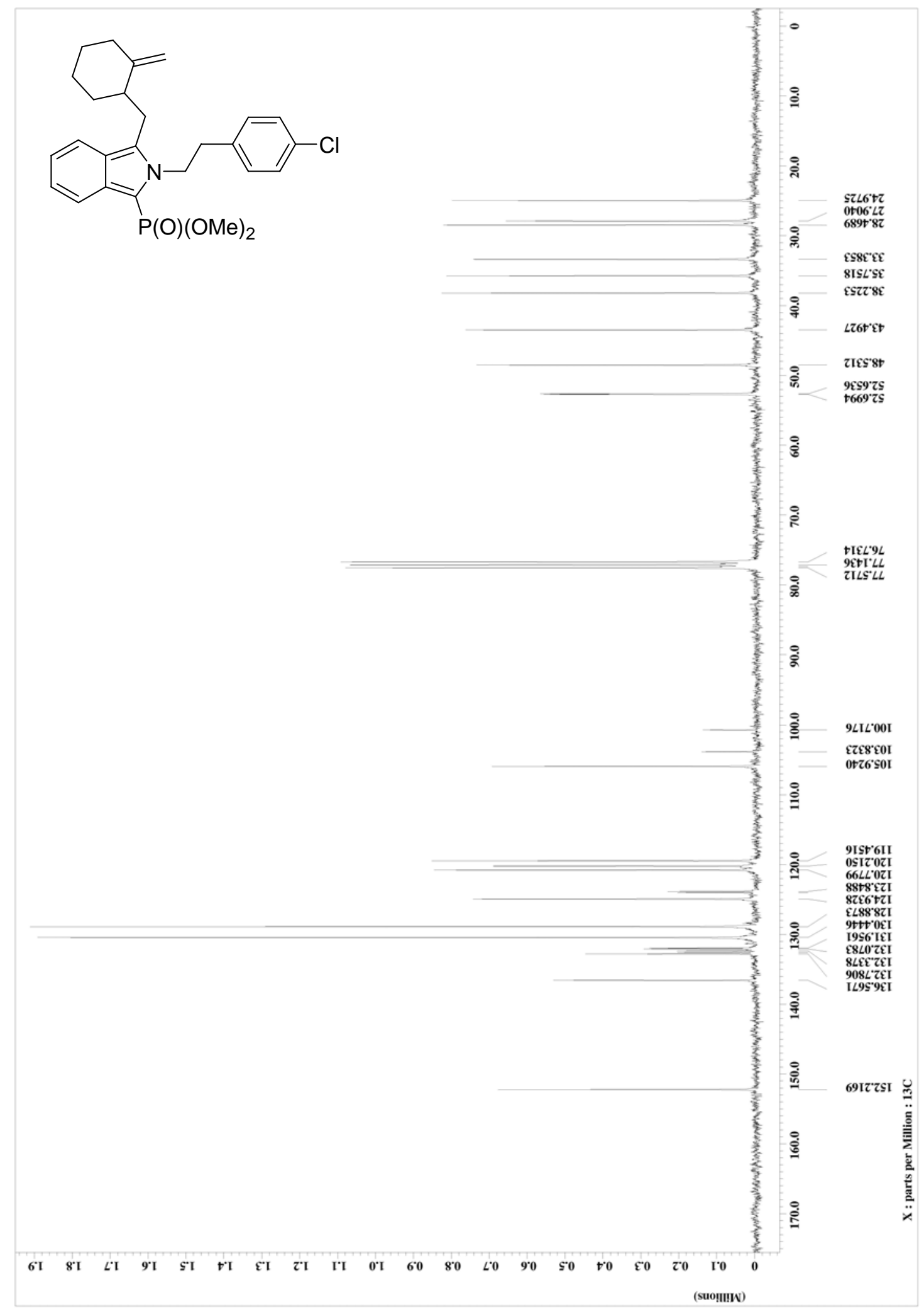


N. Dieltiens and C. V. Stevens

4g ${ }^{1} \mathrm{H}-$ Spectrum

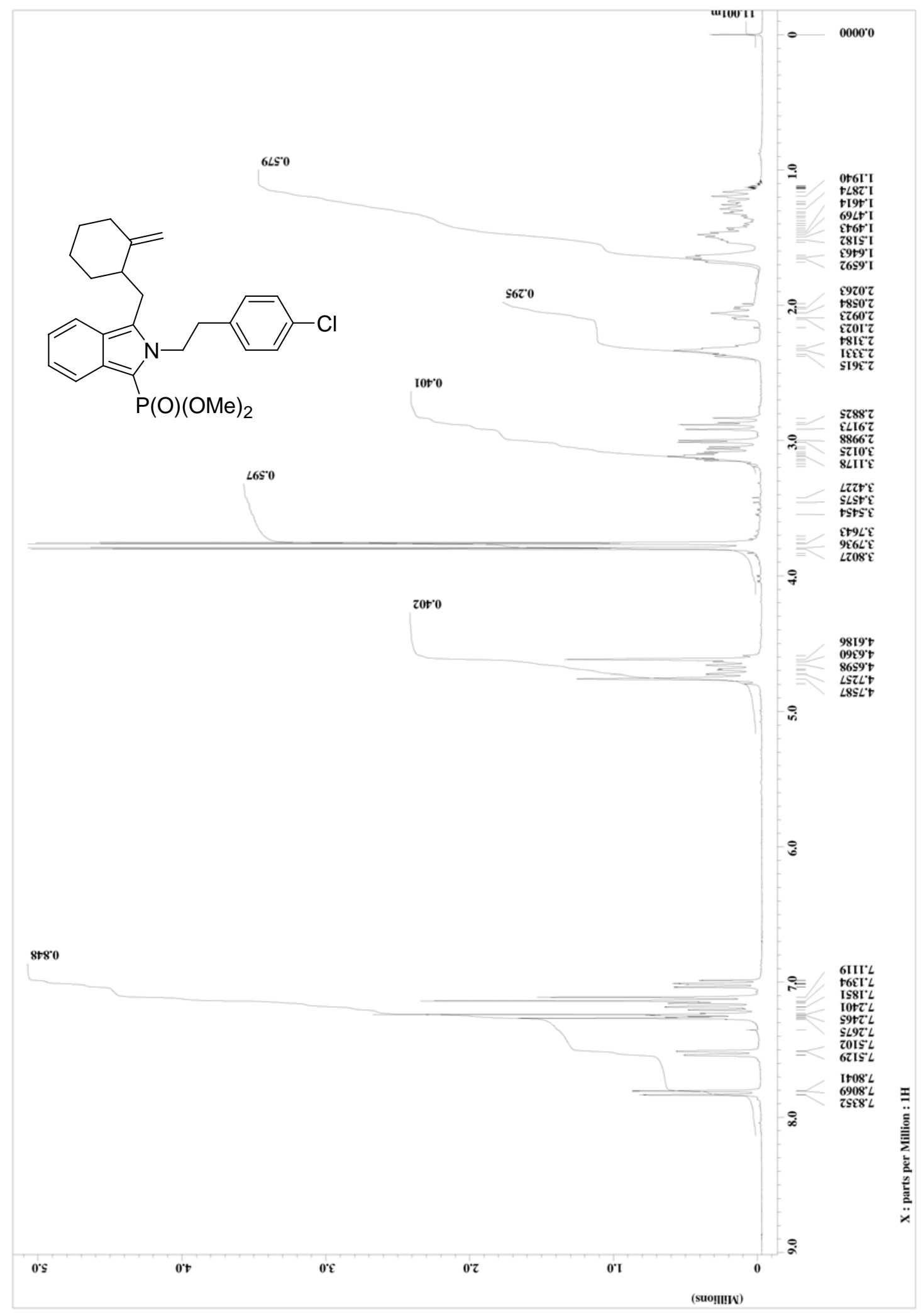




\section{4h ${ }^{13} \mathrm{C}$-Spectrum}

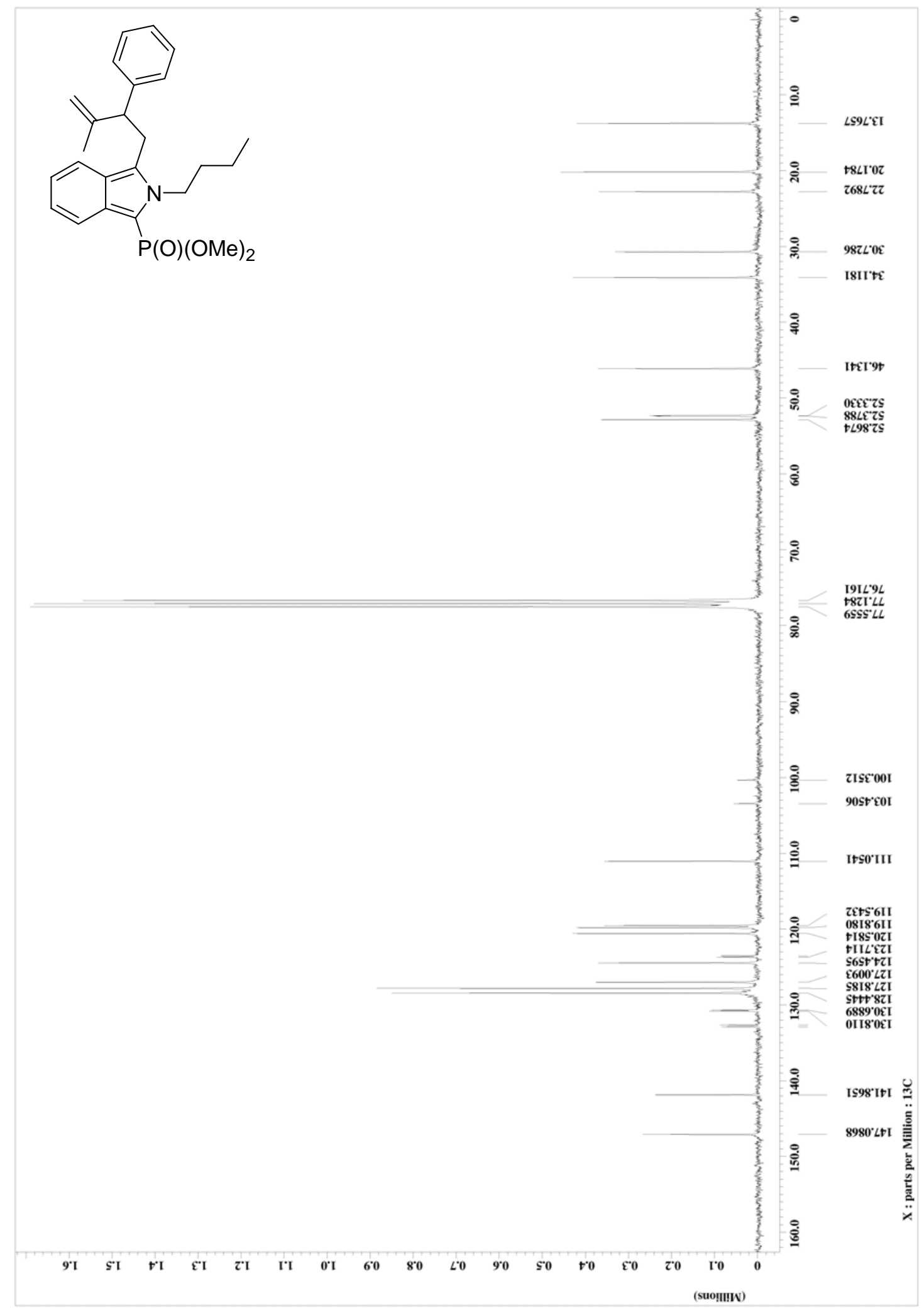


N. Dieltiens and C. V. Stevens

4h ${ }^{1} \mathrm{H}$-Spectrum

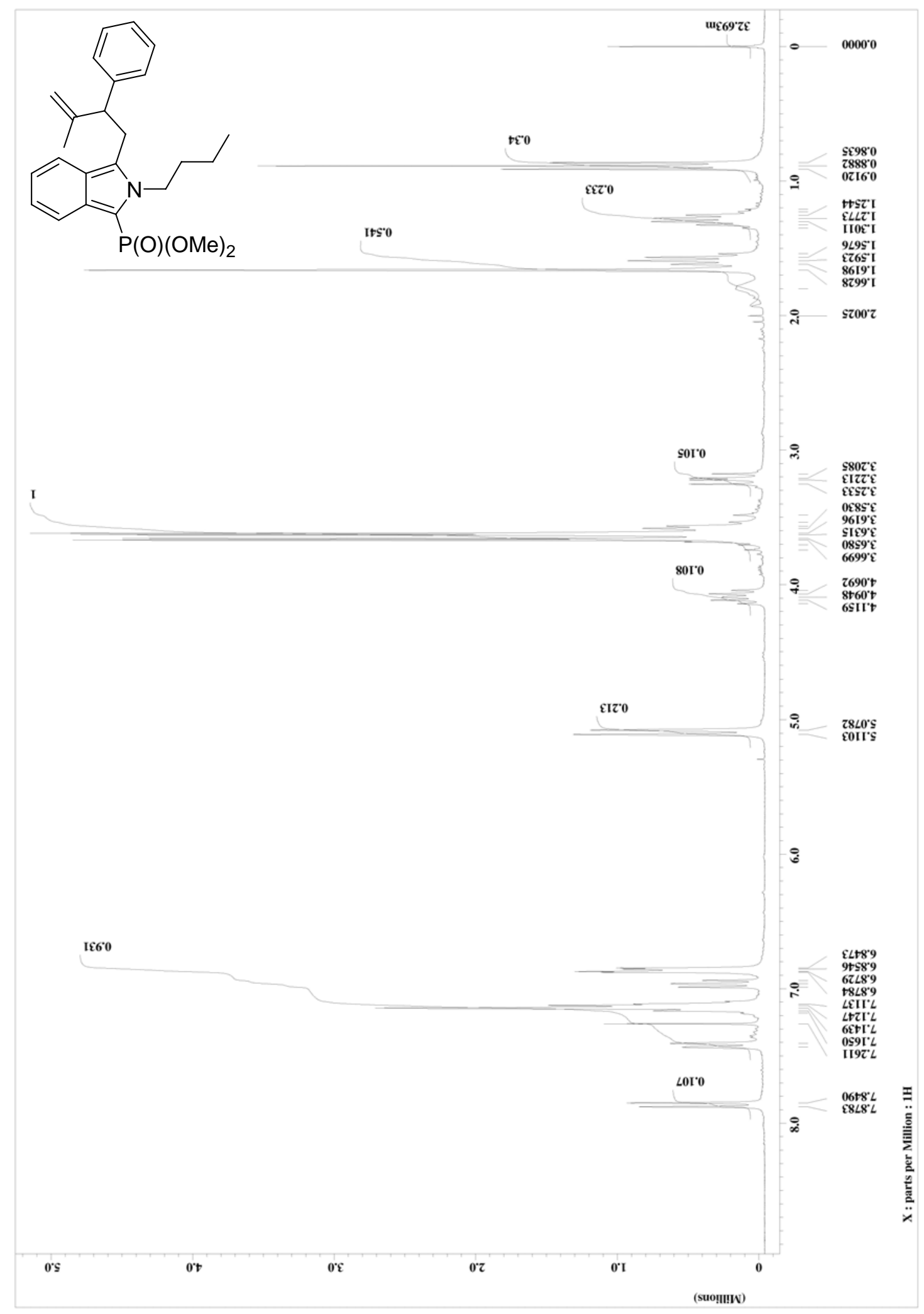


N. Dieltiens and C. V. Stevens

$4 i^{13} \mathrm{C}$-Spectrum

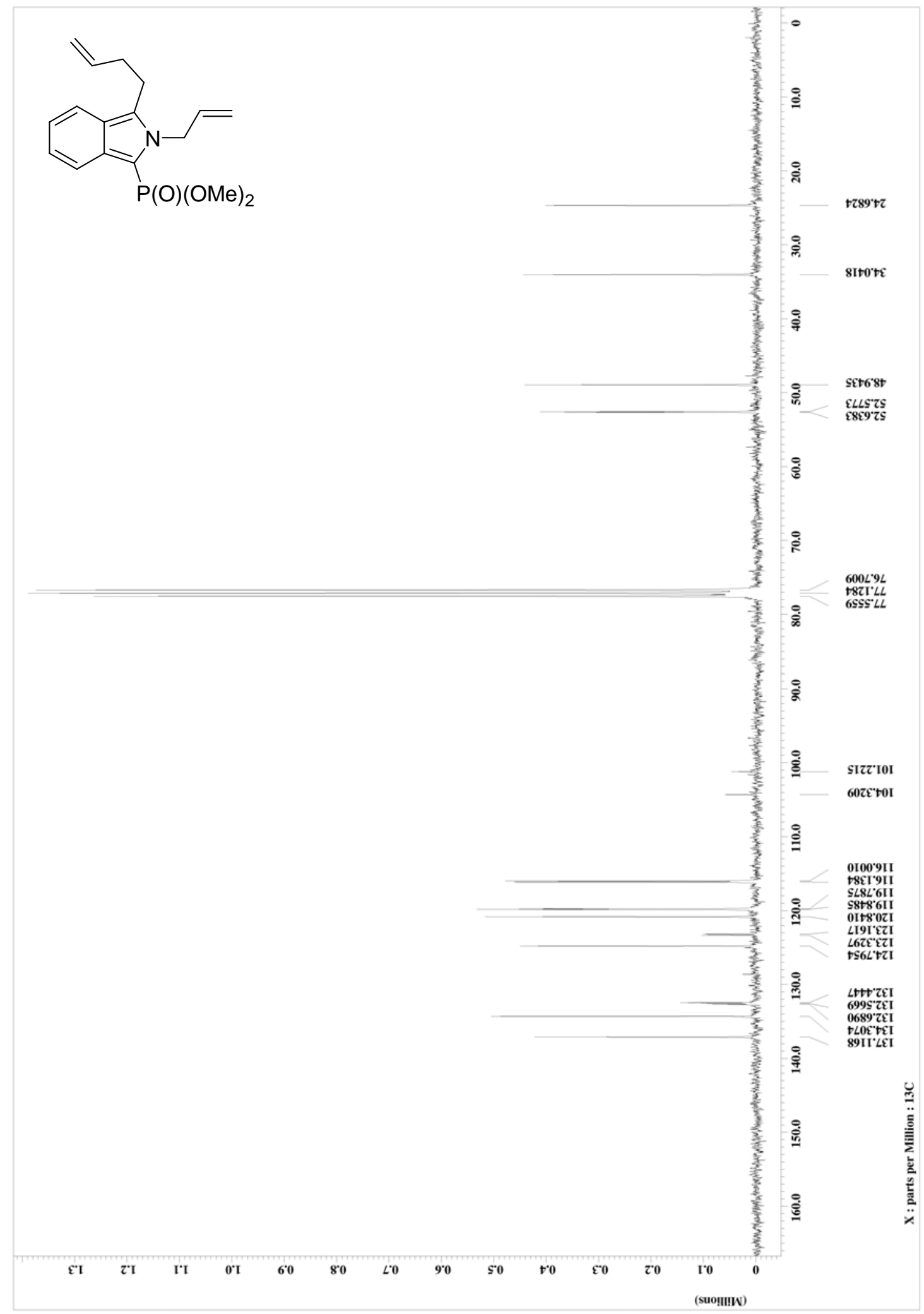


N. Dieltiens and C. V. Stevens

4i ${ }^{1} \mathrm{H}$-Spectrum

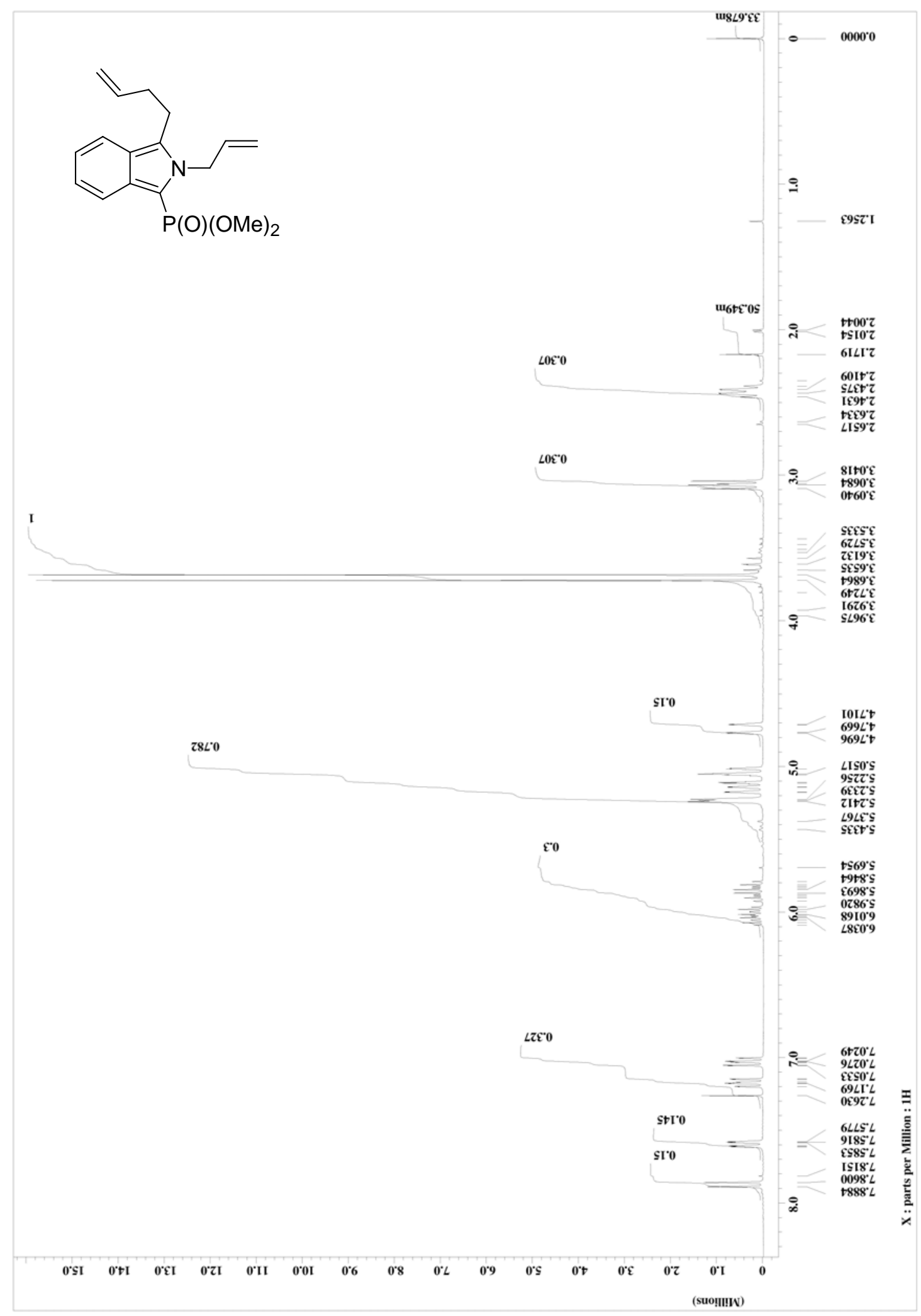


N. Dieltiens and C. V. Stevens

$13{ }^{13} \mathrm{C}-$ Spectrum

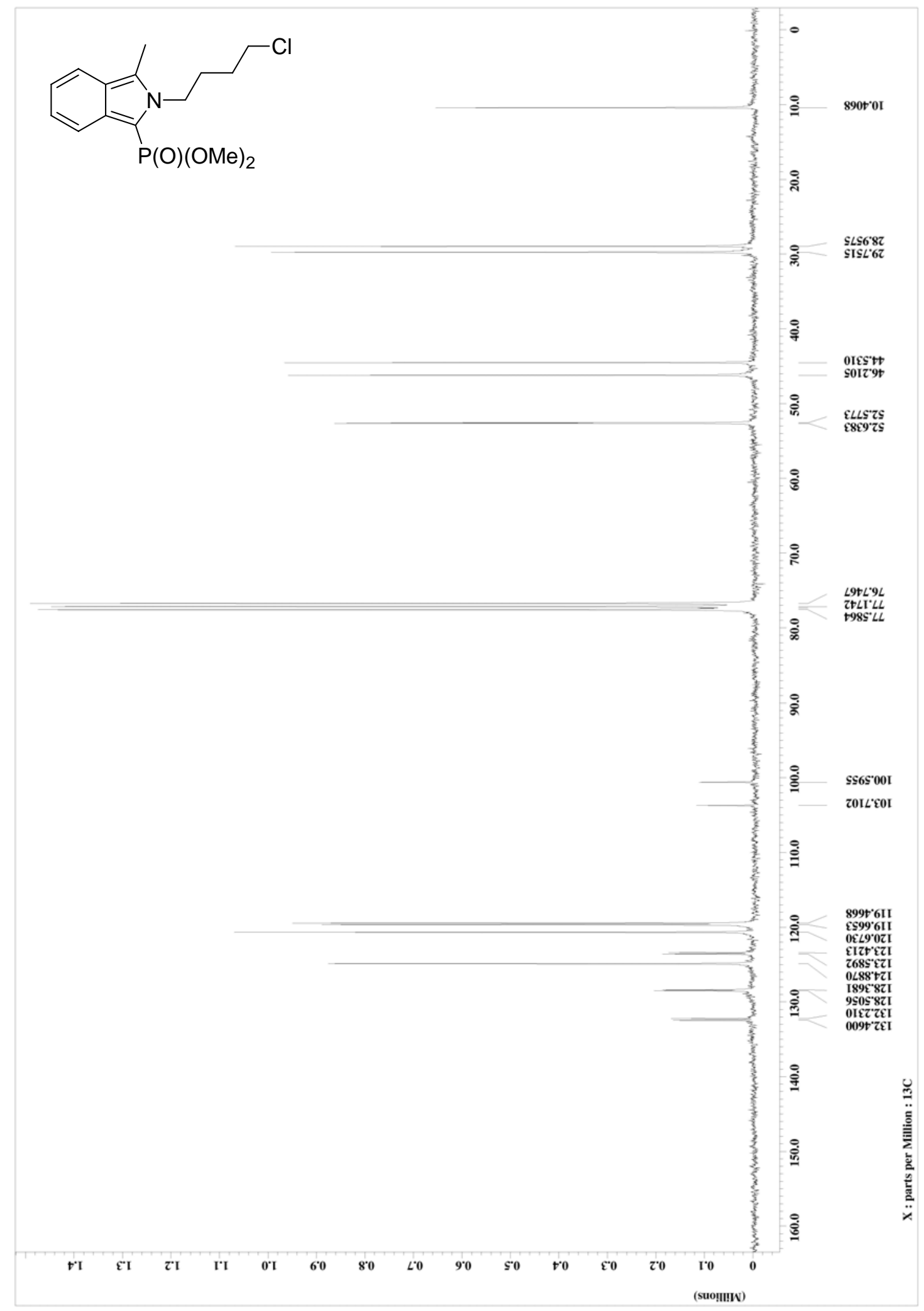


N. Dieltiens and C. V. Stevens

\section{$13{ }^{1} \mathrm{H}$-Spectrum}

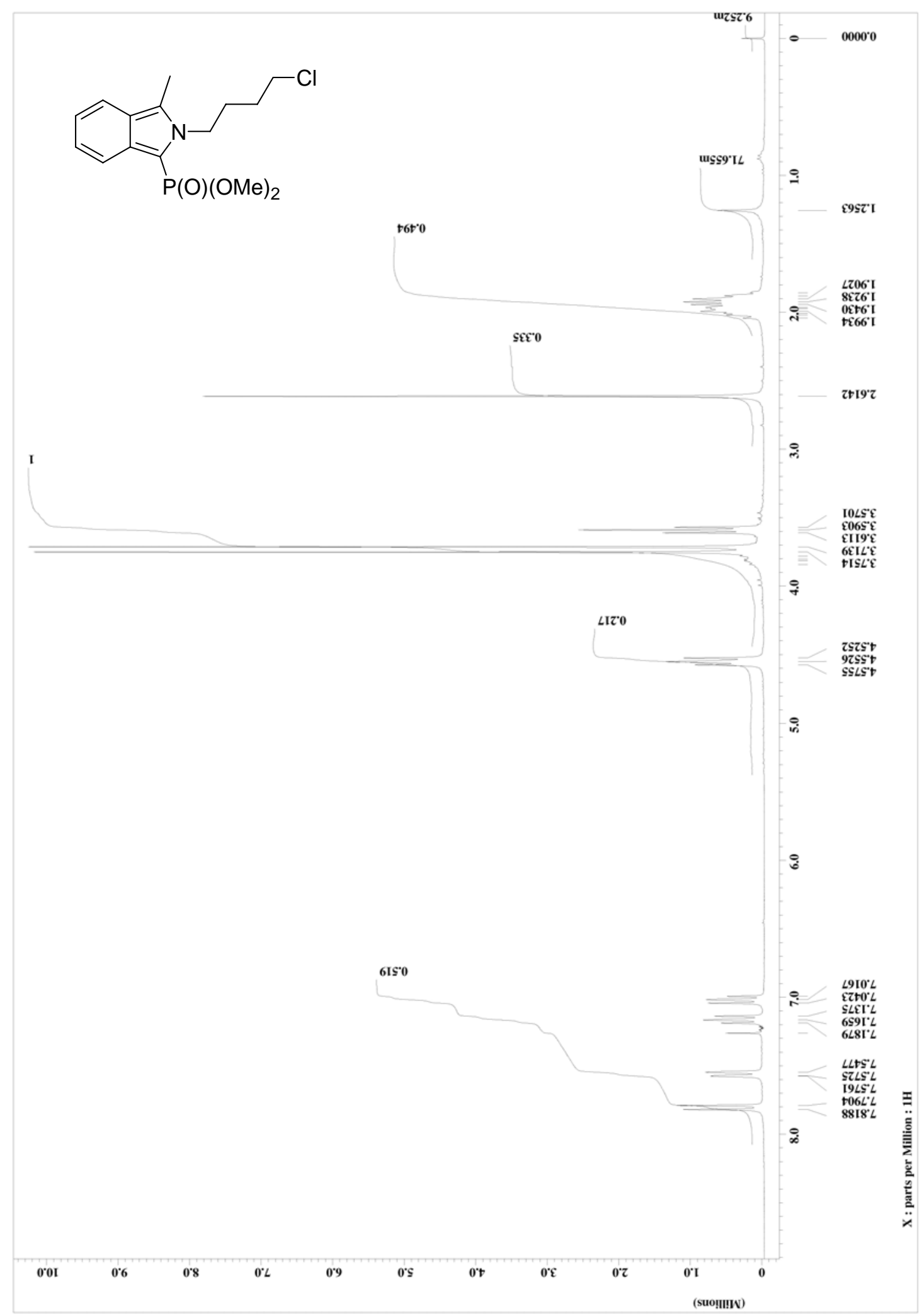


N. Dieltiens and C. V. Stevens

$\mathbf{1 4 a}{ }^{13} \mathrm{C}$-Spectrum

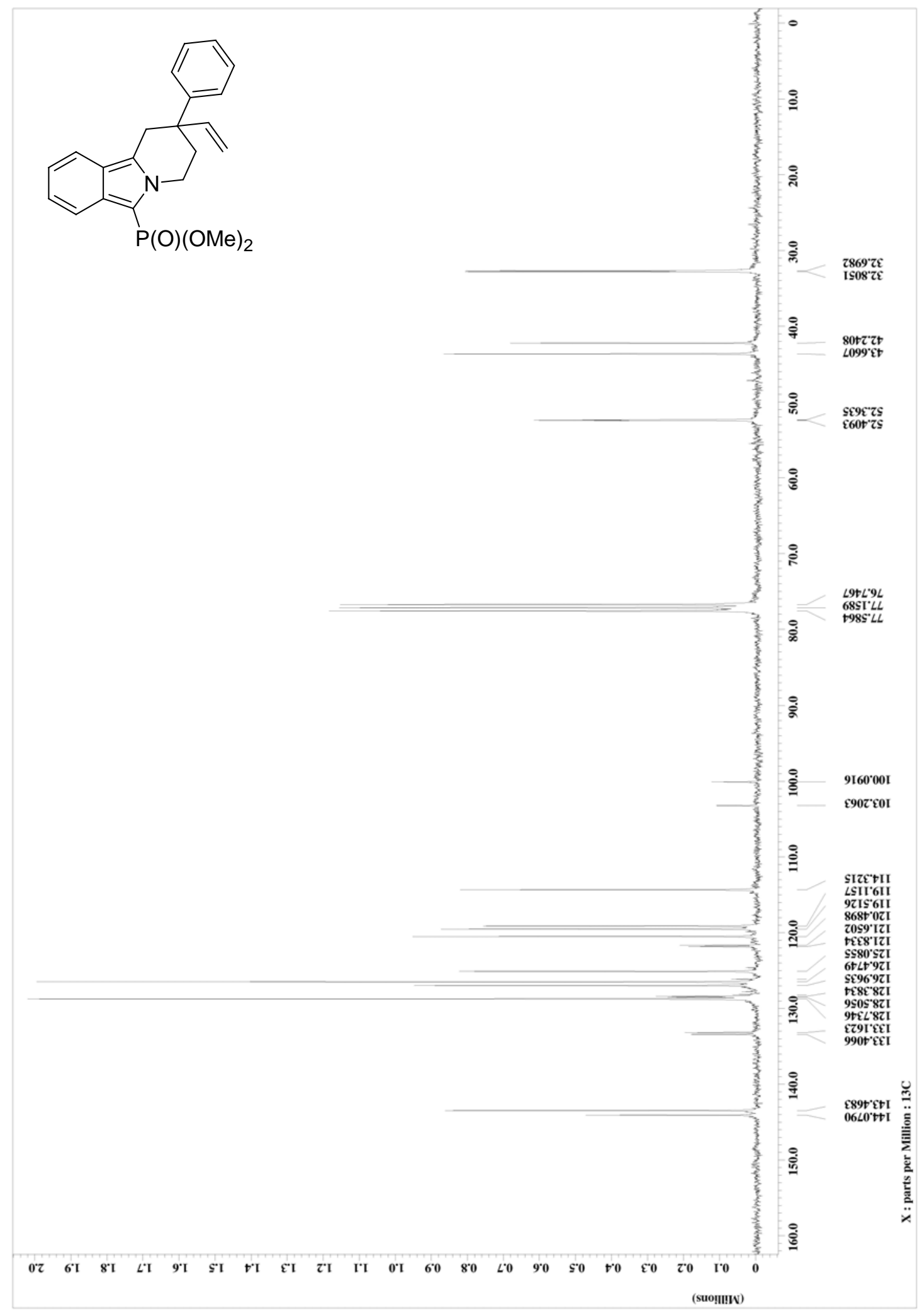


N. Dieltiens and C. V. Stevens

14a ${ }^{1} \mathrm{H}$-Spectrum

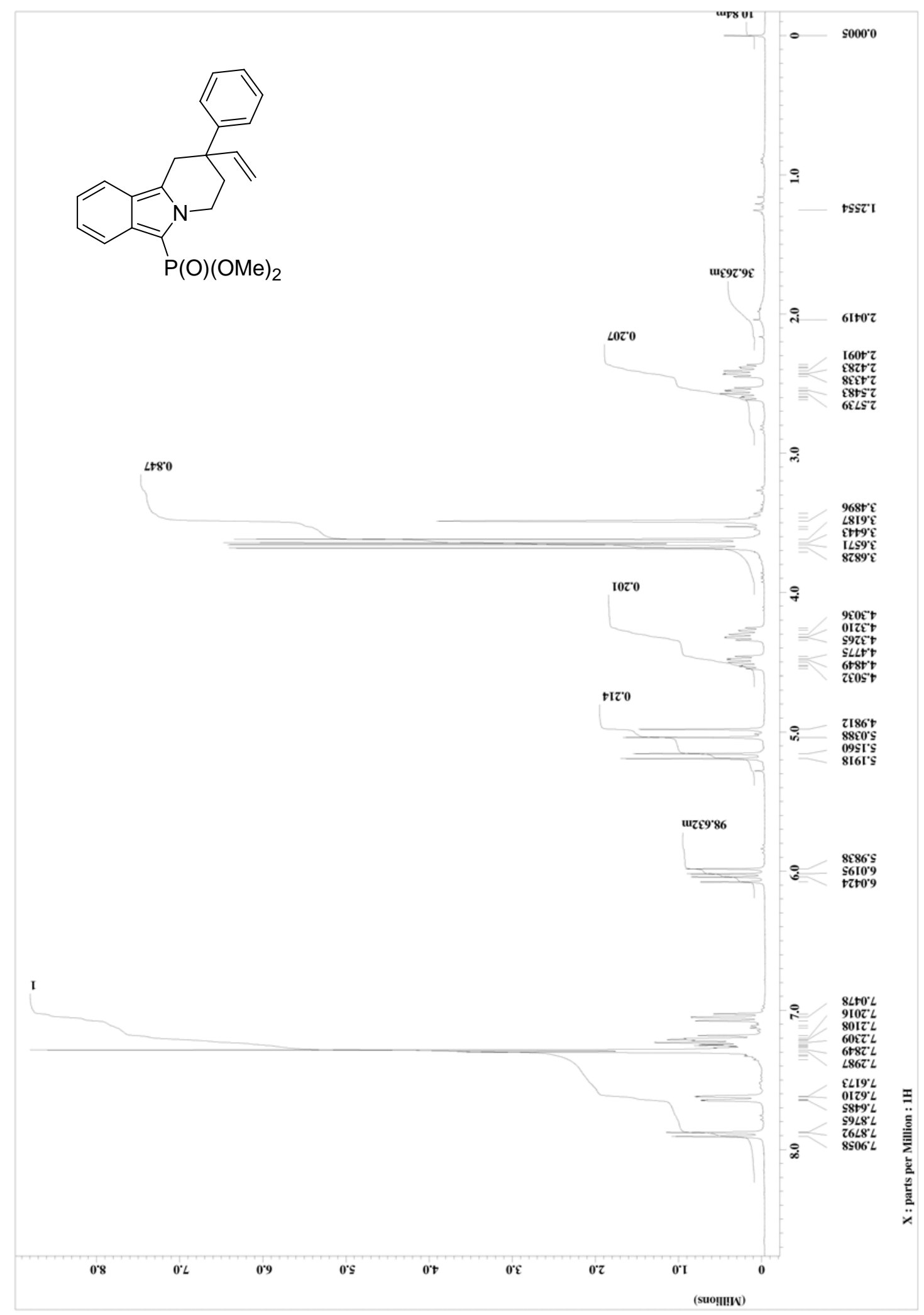


N. Dieltiens and C. V. Stevens

14b ${ }^{13}$ C-Spectrum

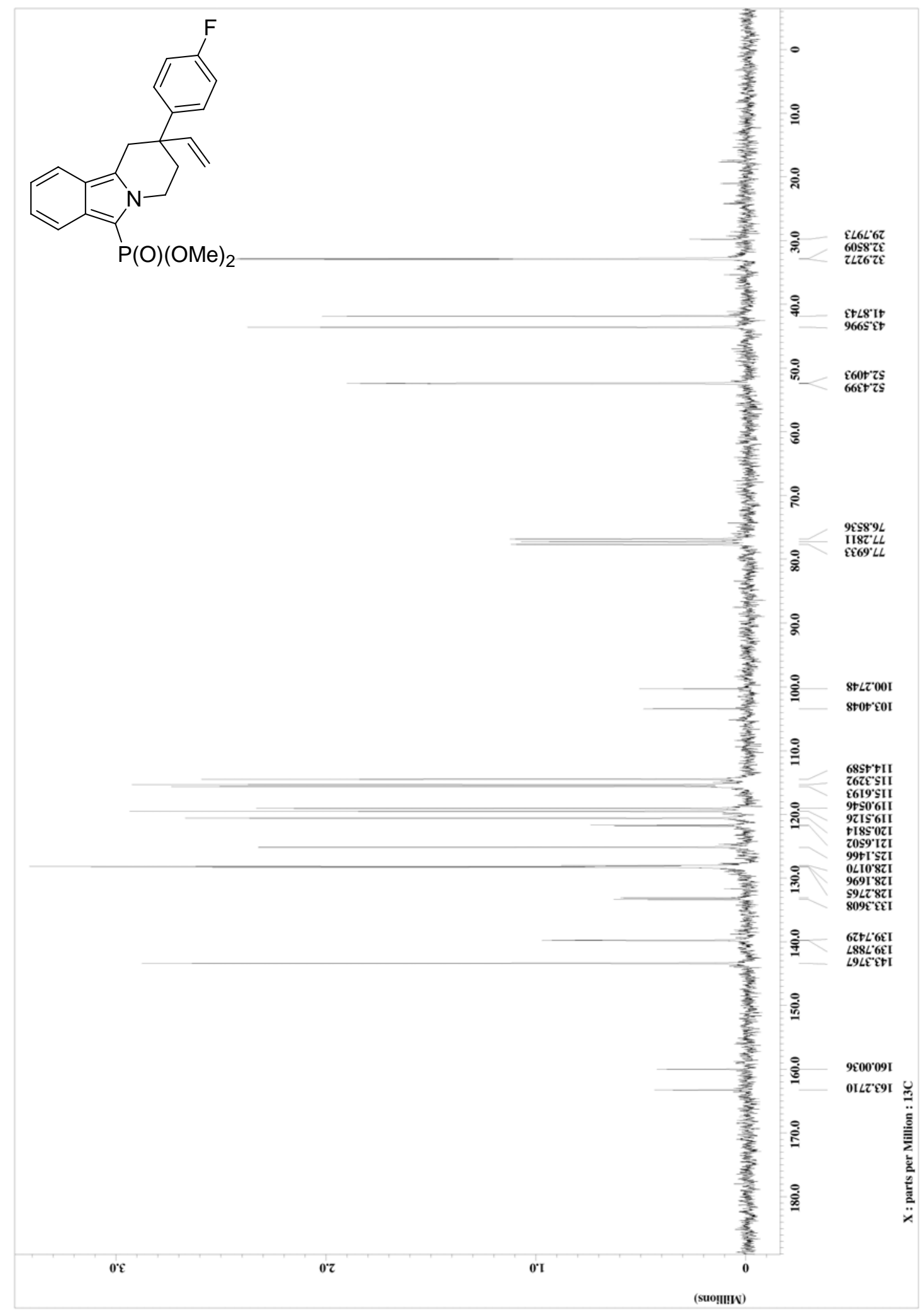


14b ${ }^{1} \mathrm{H}$-Spectrum

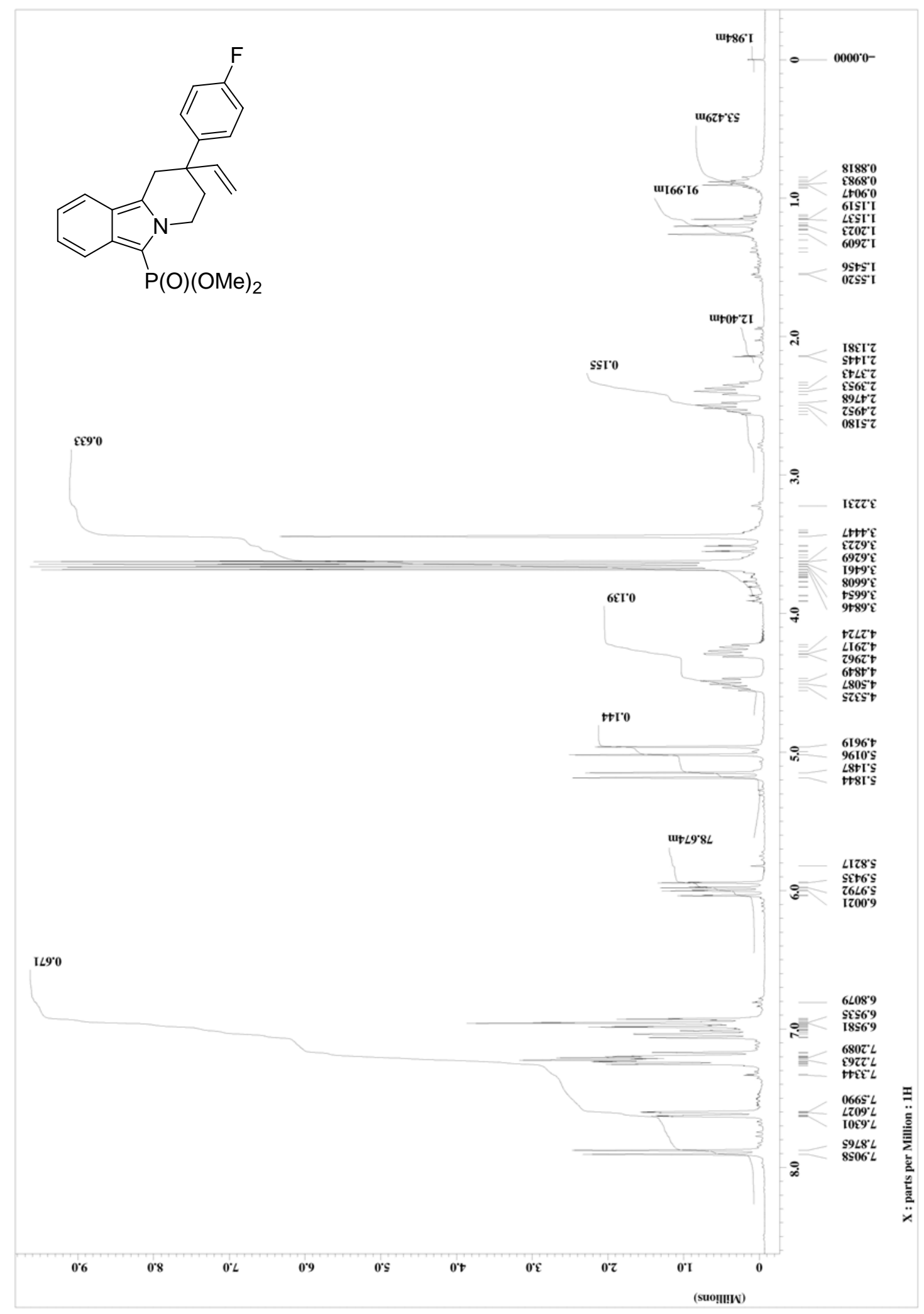


N. Dieltiens and C. V. Stevens

\section{$16{ }^{13} \mathrm{C}-$ Spectrum}

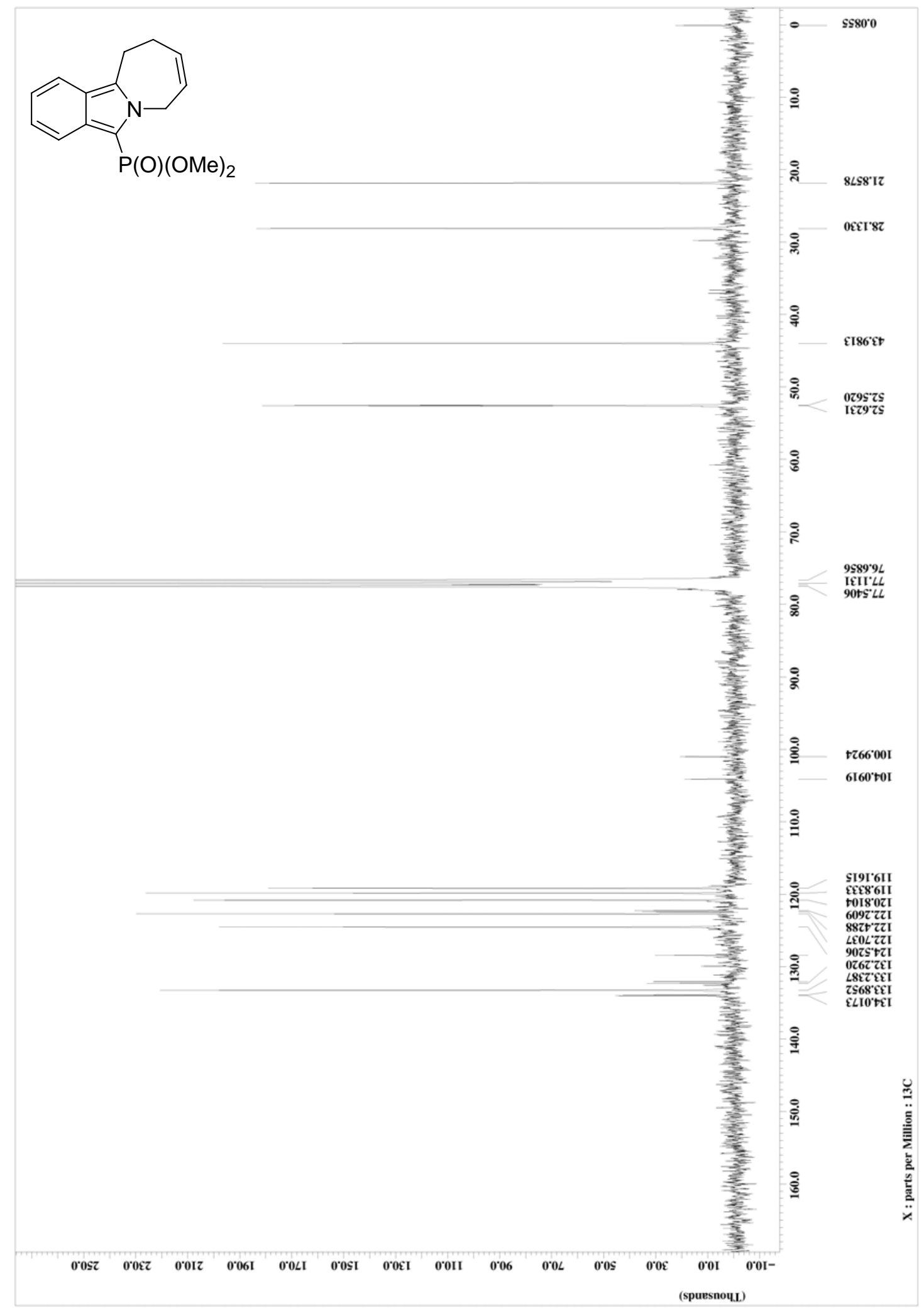


N. Dieltiens and C. V. Stevens

\section{$16{ }^{1} \mathrm{H}$-Spectrum}

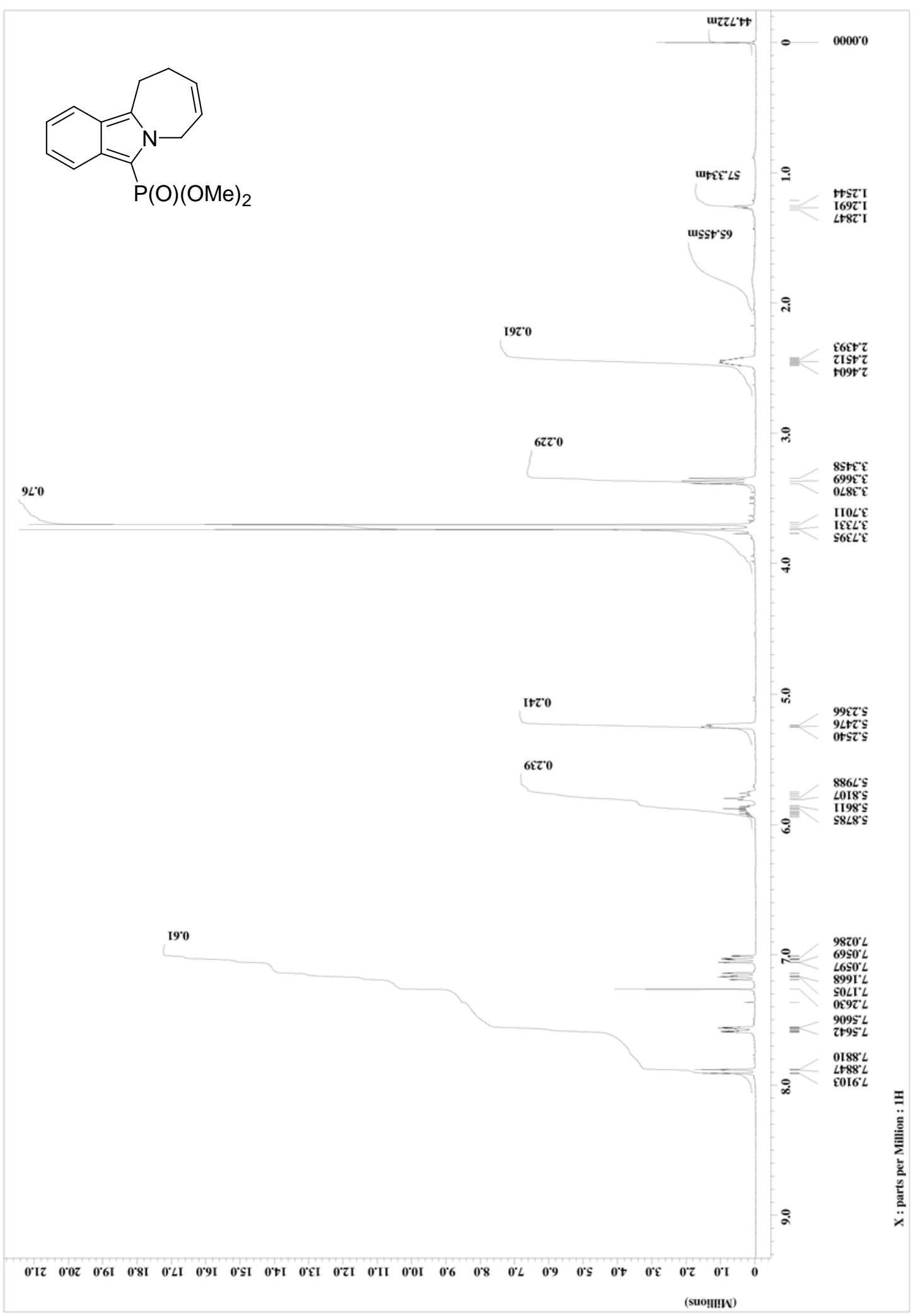

